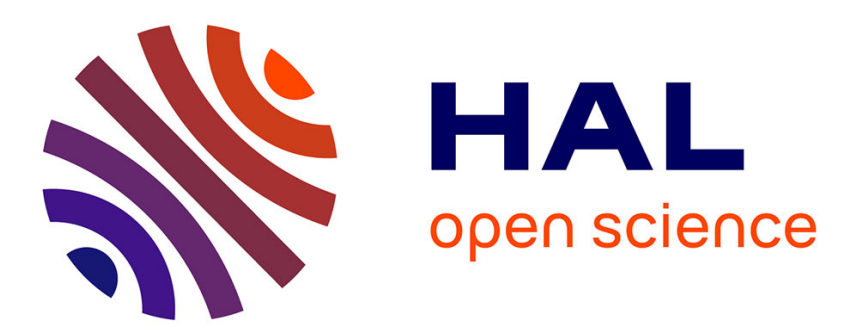

\title{
A simple a posteriori estimate on general polytopal meshes with applications to complex porous media flows
}

Martin Vohralík, Soleiman Yousef

\section{To cite this version:}

Martin Vohralík, Soleiman Yousef. A simple a posteriori estimate on general polytopal meshes with applications to complex porous media flows. Computer Methods in Applied Mechanics and Engineering, 2018, 331, pp.728-760. 10.1016/j.cma.2017.11.027 . hal-01532195v3

\section{HAL Id: hal-01532195 \\ https://hal.science/hal-01532195v3}

Submitted on 13 Feb 2018

HAL is a multi-disciplinary open access archive for the deposit and dissemination of scientific research documents, whether they are published or not. The documents may come from teaching and research institutions in France or abroad, or from public or private research centers.
L'archive ouverte pluridisciplinaire HAL, est destinée au dépôt et à la diffusion de documents scientifiques de niveau recherche, publiés ou non, émanant des établissements d'enseignement et de recherche français ou étrangers, des laboratoires publics ou privés. 


\title{
A simple a posteriori estimate on general polytopal meshes with applications to complex porous media flows*
}

\author{
Martin Vohralík $k^{\dagger} \quad$ Soleiman Yousef ${ }^{\ddagger}$
}

February 13, 2018

\begin{abstract}
This paper develops an a posteriori error estimate for lowest-order locally conservative methods on meshes consisting of general polytopal elements. We focus on the ease of implementation of the methodology based on $H^{1}$-conforming potential reconstructions and $\mathbf{H}(\operatorname{div}, \Omega)$-conforming flux reconstructions. In particular, the evaluation of our estimates for steady linear diffusion equations merely consists in some local matrix-vector multiplications, where, on each mesh element, the matrices are either directly inherited from the given numerical method, or easily constructed from the element geometry, while the vectors are the flux and potential values on the given element. We next extend our approach to steady nonlinear problems. We obtain a guaranteed upper bound on the total error in the fluxes that is still obtained by local matrix-vector multiplications, with the same element matrices as above. Moreover, the estimate holds true on any linearization and algebraic solver step and allows to distinguish the different error components. Finally, we apply this methodology to unsteady nonlinear coupled degenerate problems describing complex multiphase flows in porous media. Also here, on each step of the time-marching scheme, linearization procedure, and linear algebraic solver, the estimate takes the simple matrix-vector multiplication form and distinguishes the different error components. It leads to an easy-to-implement and fast-to-run adaptive algorithm with guaranteed overall precision, adaptive stopping criteria, and adaptive space and time mesh refinements. Numerous numerical experiments on practical problems in two and three space dimensions illustrate the performance of our methodology.
\end{abstract}

Key words: A posteriori error estimate, polytopal meshes, stopping criteria, adaptivity, porous media flow

\section{Introduction}

The use of general polygonal/polyhedral meshes (we henceforth use the term polytopal for any space dimension) is very appealing in various domains in computational practice. In these last years, there has been a growing mathematical background for a priori analysis (existence, uniqueness, convergence, a priori error estimates), see, e.g., Brezzi et al. [16, 17], Wheeler and Yotov [68], Aavatsmark et al. [2], Droniou et al. [34, 35], Beirão da Veiga et al. [8, 9], Vohralík and Wohlmuth [67], Bonelle and Ern [13], Cangiani et al. [21], Cockburn et al. [27], and the references therein.

Literature on a posteriori analysis on polytopal meshes is much less plentiful. Beirão da Veiga [7] and Beirão da Veiga and Manzini [10] derive a posteriori error estimates for low-order mimetic finite difference methods; extension to higher-order is presented in Antonietti et al. [5]. Arbitrary-order discontinuous Galerkin methods are analysed in Ern and Vohralík [38], relying on the concept of a simplicial submesh. Similarly, Vohralík and Wohlmuth [67] cover at once all the mimetic finite difference, mixed finite volume, hybrid finite volume, and mixed finite element methods on general polytopal meshes. Beirão da Veiga and Manzini [11] as well as Cangiani et al. [22] then derive a posteriori error estimates for the virtual element

\footnotetext{
*This project has partially received funding from the European Research Council (ERC) under the European Union's Horizon 2020 research and innovation program (grant agreement No 647134 GATIPOR).

${ }^{\dagger}$ Inria Paris, 2 rue Simone Iff, 75589 Paris, France \& Université Paris-Est, CERMICS (ENPC), 77455 Marne-la-Vallée, France (martin.vohralik@inria.fr).

${ }^{\ddagger}$ IFP Energies nouvelles, 1 \& 4 av. Bois Préau, 92852 Rueil-Malmaison, France (soleiman.yousef@ifpen.fr).
} 
method. Among related results, one could mention Buffa and Giannelli [18] who study the isogeometric methods, cf., e.g., Bazilevs et al. [6].

The purpose of the present contribution is to derive simple a posteriori error estimates applicable on general polytopal meshes. Developing the ideas from $[38,67]$, we do so in a framework including any lowestorder locally conservative method like the mimetic finite difference [16, 17], multi-point finite volume [1, 2, 14, 37], mixed finite volume [33], hybrid finite volume [42], multipoint flux mixed finite element [68], mixed virtual element [9], or mixed finite element $[24,52,53,61,67]$ ones, see also $[12,13,23,32,35,47,51]$ and the references therein. Higher-order methods could be treated similarly. Our focus is to derive estimates that can be easily coded, cheaply evaluated, and efficiently used in practical simulations. In particular, we want to avoid, if possible, the physical construction and coding of a simplicial submesh and solution of local problems. The evaluation of our estimates is fully explicit and merely consists in multiplication of the vectors collecting flux and potential values on each polytope $K$ by the some local matrices associated with $K$. This probably gives the easiest and most practically accessible application to polytopal meshes of the general methodology of $H^{1}$-conforming potential reconstruction and $\mathbf{H}(\operatorname{div}, \Omega)$-conforming flux reconstruction, see Ern and Vohralík [40] and the references therein. In the first part of the paper, namely in Section 3, we give all the details for a model steady linear diffusion problem, after having set the notation in Section 2. Numerical illustration for this case forms a content of Section 4.

In the second part of our paper, in Section 5, we show that structurally the same results can be obtained for a model nonlinear steady diffusion problem. The estimators still take the form of a simple matrix-vector multiplication, with the same local matrices as in the linear case. The guaranteed upper bound on the error in the fluxes in addition allows to identify the parts corresponding to the three arising error components because of discretization, linearization, and algebraic solver.

In the third and last part of our paper, we apply the entire methodology to the context of numerical approximation of unsteady nonlinear systems of partial differential equations describing the multiphase porous media Darcy flow. The estimates derived in Section 6 are still fully computable and feature no unknown generic constant. They are crucially valid at each stage of the overall solution algorithm: on each time step $n$, each linearization step $k$, and each linear solver step $i$. They also allow to distinguish different error components and design adaptive stopping criteria for the involved iterative solvers, as well as adaptive choice of space and time meshes. A detailed numerical study in this case forms the content of Section 7. Finally, conclusions of our paper are drawn in Section 8.

\section{Setting}

Let $\Omega \subset \mathbb{R}^{d}, d \geq 1$, be an open interval/polygon/polyhedron for respectively $d=1,2,3$, or a polytope in general, with a Lipschitz-continuous boundary $\partial \Omega$. We are interested in partitions $\mathcal{T}_{H}$ of the domain $\Omega$ into polytopes $K$ such that $\cup_{K \in \mathcal{T}_{H}} K=\bar{\Omega}$. The elements $K$ can in particular be nonconvex and the mesh $\mathcal{T}_{H}$ can be nonmatching in the sense that intersection of two neighboring polytopal elements is not necessarily their entire face. The only assumption we impose is that there exists a (virtual, not necessarily to be constructed in practice) simplicial submesh $\mathcal{T}_{h}$ of $\mathcal{T}_{H}$ which is matching, shape-regular, and such that any polytopal element $K \in \mathcal{T}_{H}$ is covered by a patch of simplices $\mathcal{T}_{K}$. In the present work, for simplicity of exposition and of the developed formulas, we suppose that there is one point inside each $K \in \mathcal{T}_{H}$ shared by all the simplices in $\mathcal{T}_{K}$, as well as that the boundary faces of the simplices in $\mathcal{T}_{K}$ create no new vertices on $\partial K$. Relaxing these two constraints, we could also allow for unlimited maximal number of faces of $K \in \mathcal{T}_{H}$, no condition on $\mathcal{T}_{H}$ to be shape-regular, and non star-shaped elements. Altogether, each element $\kappa \in \mathcal{T}_{h}$ is a simplex, $\cup_{\kappa \in \mathcal{T}_{h}} \kappa=\bar{\Omega}, \cup_{\kappa \in \mathcal{T}_{K}} \kappa=K, \cup_{K \in \mathcal{T}_{H}} \mathcal{T}_{K}=\mathcal{T}_{h}$, the intersection of two elements in $\mathcal{T}_{h}$ is either empty or their $d^{\prime}$-dimensional common face, $0 \leq d^{\prime} \leq d-1$, and the ratios of the diameter of each $\kappa \in \mathcal{T}_{h}$ to the diameter of its largest inscribed ball are uniformly bounded by a constant $\varrho_{\mathcal{T}}>0$ for all considered meshes $\left\{\mathcal{T}_{H}\right\}_{H}$. An illustration in two dimensions is provided in Figure 1.

Let $\mathcal{E}_{H}$ be the set of the $(d-1)$-dimensional faces of $\mathcal{T}_{H}$, divided into interior faces $\mathcal{E}_{H}^{\text {int }}$ and boundary faces $\mathcal{E}_{H}^{\text {ext }}: \sigma \in \mathcal{E}_{H}^{\text {int }}$ if there exist $K, K^{\prime} \in \mathcal{T}_{H}, K \neq K^{\prime}$, such that $\sigma=K \cap K^{\prime}$, and $\sigma \in \mathcal{E}_{H}^{\text {ext }}$ if there exist $K \in \mathcal{T}_{H}$ such that $\sigma=K \cap \partial \Omega$. Similarly, let $\mathcal{E}_{h}$ be the set of the $(d-1)$-dimensional faces of the simplicial mesh $\mathcal{T}_{h}$. We denote by $\mathcal{E}_{H, h}$ the set of such faces from $\mathcal{E}_{h}$ that lie in some polytopal face of $\mathcal{E}_{H}$. If the faces of $\mathcal{T}_{h}$ do not subdivide the faces of $\mathcal{T}_{H}$, then $\mathcal{E}_{H, h}=\mathcal{E}_{H}$. Let $\mathcal{E}_{K} \subset \mathcal{E}_{H}$ be the set of the $(d-1)$-dimensional faces of the polytope $K \in \mathcal{T}_{H}$, and let $\mathcal{E}_{K, h}^{\text {ext }}$ collect those faces of $\mathcal{E}_{H, h}$ that lie on the boundary of the element 


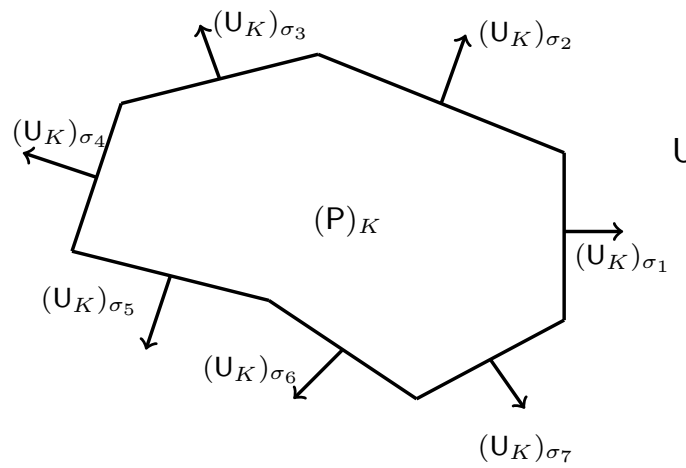

$$
\begin{gathered}
\mathcal{E}_{K}=\left\{\sigma_{i}\right\}_{i=1}^{7} \\
\mathrm{U}_{K}^{\mathrm{ext}}=\left\{\left(\mathrm{U}_{K}\right)_{\sigma_{i}}\right\}_{i=1}^{7} \\
\mathcal{T}_{K}=\left\{\kappa_{i}\right\}_{i=1}^{7} \\
\mathcal{E}_{K, h}^{\mathrm{ext}}=\left\{\sigma_{i}\right\}_{i=1}^{7} \\
\mathcal{E}_{K, h}^{\mathrm{int}}=\left\{\sigma_{i}\right\}_{i=8}^{14} \\
\mathcal{E}_{K, h}=\mathcal{E}_{K, h}^{\mathrm{ext}} \cup \mathcal{E}_{K, h}^{\mathrm{int}}
\end{gathered}
$$

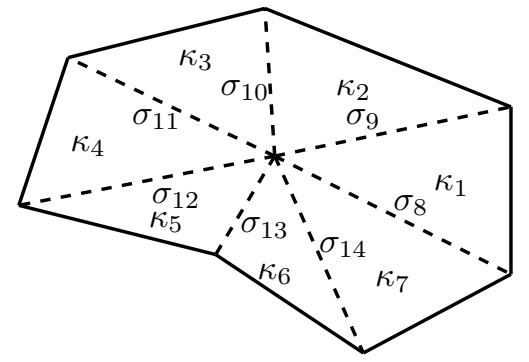

Figure 1: Example of a general polygonal element $K$ with its faces $\mathcal{E}_{K}$, corresponding face fluxes $\cup_{K}^{\text {ext }}$, and pressure head $(\mathrm{P})_{K}$ (left); virtual simplicial submesh $\mathcal{T}_{K}$ of $K$ (right)

$K \in \mathcal{T}_{H}$, whereas $\mathcal{E}_{K, h}^{\text {int }}$ those faces of $\mathcal{E}_{H, h}$ that lie inside the element $K \in \mathcal{T}_{H}$, see Figure 1 . To complete the notation, we also let $\mathcal{V}_{h}$ be the set of the vertices of the mesh $\mathcal{T}_{h}, \mathcal{V}_{\sigma}$ vertices of the face $\sigma \in \mathcal{E}_{h}, \mathcal{V}_{K}$ vertices of the element $K \in \mathcal{T}_{H}$, and $\mathcal{V}_{K, h}$ the vertices of all the elements $\kappa \in \mathcal{T}_{K}$.

For a set $\mathcal{S},|\mathcal{S}|$ henceforth stands for its cardinality. We also use the notation $|K|$ for the Lebesgue measure of an element $K \in \mathcal{T}_{H},|\sigma|$ for the $(d-1)$-dimensional Lebesgue measure of a face $\sigma \in \mathcal{E}_{H}$, and $|\mathbf{v}|$ for the (Euclidean) size of a vector $\mathbf{v} \in \mathbb{R}^{d}$. For an interior face $\sigma \in \mathcal{E}_{H}^{\text {int }}$, we fix an arbitrary orientation and denote the corresponding unit normal vector by $\mathbf{n}_{\sigma}$. For a boundary face $\sigma \in \mathcal{E}_{H}^{\text {ext }}, \mathbf{n}_{\sigma}$ coincides with the exterior unit normal $\mathbf{n}_{\Omega}$ of $\Omega$. Finally, for all $K \in \mathcal{T}_{H}$ and all $\sigma \in \mathcal{E}_{K}$, we denote by $\mathbf{n}_{K, \sigma}$ the unit normal vector to $\sigma$ pointing out of $K$.

\section{A steady linear problem}

We describe here first a model steady linear problem together with its generic locally conservative polytopal discretization. Then our a posteriori estimates, relying on the methodology of flux and potential reconstructions where we avoid their factual construction, are described.

\subsection{Single-phase Darcy flow}

Consider the problem of finding $p: \Omega \rightarrow \mathbb{R}$ such that

$$
\begin{aligned}
-\nabla \cdot(\underline{\mathbf{K} \nabla p)}=f & \text { in } \Omega, \\
p=0 & \text { on } \partial \Omega .
\end{aligned}
$$

Here $f$ is a source term and $\underline{\mathbf{K}}$ is a symmetric, positive definite, and bounded diffusion tensor with values in $\mathbb{R}^{d \times d}$; we suppose for simplicity that both $f$ and $\underline{\mathbf{K}}$ are piecewise constant with respect to the mesh $\mathcal{T}_{H}$. In weak form, (3.1) amounts to looking for $p \in H_{0}^{1}(\Omega)$ such that

$$
(\underline{\mathbf{K}} \nabla p, \nabla v)=(f, v) \quad \forall v \in H_{0}^{1}(\Omega) .
$$

From the pressure head $p$, we can define the Darcy velocity

$$
\mathbf{u}:=-\underline{\mathbf{K}} \nabla p
$$

we also refer to $p$ as potential and to $\mathbf{u}$ as flux. It follows from (3.2) that $\mathbf{u}$ lies in $\mathbf{H}(\operatorname{div}, \Omega)$ and is such that $\nabla \cdot \mathbf{u}=f$. In the sequel, we will often use the local energy norm on $K \in \mathcal{T}_{H}$; for $\mathbf{v} \in\left[L^{2}(K)\right]^{d}$,

$$
\|\mathbf{v}\|_{\underline{\mathbf{K}}^{-\frac{1}{2} ; L^{2}(K)}}:=\left\|\underline{\mathbf{K}}^{-\frac{1}{2}} \mathbf{v}\right\|_{L^{2}(K)}=\left\{\int_{K}\left|\underline{\mathbf{K}}^{-\frac{1}{2}}(\mathbf{x}) \mathbf{v}(\mathbf{x})\right|^{2} \mathrm{~d} \mathbf{x}\right\}^{\frac{1}{2}} .
$$




\subsection{Generic discretizations on a polytopal mesh}

Let $\mathcal{T}_{H}$ be a polytopal mesh of $\Omega$ satisfying the assumptions of Section 2 and define a vector $\mathrm{F} \in \mathbb{R}^{\left|\mathcal{T}_{H}\right|}$ by

$$
(\mathrm{F})_{K}:=(f, 1)_{K} \quad \forall K \in \mathcal{T}_{H} .
$$

We consider in this paper any scheme that can be written under the following abstract form:

Assumption 3.1 (Locally conservative discretization on a polytopal mesh). Find $\mathrm{U} \in \mathbb{R}^{\left|\mathcal{E}_{H}\right|}$ and $\mathrm{P} \in \mathbb{R}^{\left|\mathcal{T}_{H}\right|}$ such that the flux balance

$$
\sum_{\sigma \in \mathcal{E}_{K}}(\mathrm{U})_{\sigma} \mathbf{n}_{K, \sigma} \cdot \mathbf{n}_{\sigma}=(\mathrm{F})_{K} \quad \forall K \in \mathcal{T}_{H}
$$

is satisfied. Here $\mathrm{U}:=\left\{(\mathrm{U})_{\sigma}\right\}_{\sigma \in \mathcal{E}_{H}}$, and the unknown $(\mathrm{U})_{\sigma} \in \mathbb{R}$ for each face $\sigma \in \mathcal{E}_{H}$ approximates the normal flux $\left\langle\mathbf{u} \cdot \mathbf{n}_{\sigma}, 1\right\rangle_{\sigma}$ over the face $\sigma$. Similarly, $\mathrm{P}:=\left\{(\mathrm{P})_{K}\right\}_{K \in \mathcal{T}_{H}}$, and the unknown $(\mathrm{P})_{K} \in \mathbb{R}$ for each element $K \in \mathcal{T}_{H}$ represents the pressure head $p$ in the element $K$.

Many lowest-order locally conservative methods take a more specific form, contained in Assumption 3.1. We consider them separately under the following assumption (see in particular [67, Theorems 7.2 and 7.3] for the relation to mixed finite elements):

Assumption 3.2 (Saddle-point discretization on a polytopal mesh). Find $\mathrm{U}:=\left\{(\mathrm{U})_{\sigma}\right\}_{\sigma \in \mathcal{E}_{H}} \in \mathbb{R}^{\left|\mathcal{E}_{H}\right|}$ and $\mathrm{P}:=\left\{(\mathrm{P})_{K}\right\}_{K \in \mathcal{T}_{H}} \in \mathbb{R}^{\left|\mathcal{T}_{H}\right|}$ such that

$$
\left(\begin{array}{ll}
\mathbb{A} & \mathbb{B}^{\mathrm{t}} \\
\mathbb{B} & 0
\end{array}\right)\left(\begin{array}{l}
\mathrm{U} \\
\mathrm{P}
\end{array}\right)=\left(\begin{array}{c}
0 \\
-\mathrm{F}
\end{array}\right)
$$

where 1) $\mathbb{B}$ has a full rank; 2) for $K \in \mathcal{T}_{H}$ and $\sigma \in \mathcal{E}_{H}, \mathbb{B}_{K, \sigma}=-\mathbf{n}_{K, \sigma} \cdot \mathbf{n}_{\sigma}$ if $\sigma \in \mathcal{E}_{K}$ and $\mathbb{B}_{K, \sigma}=0$ otherwise; 3) $\mathbb{A}$ is square and invertible; 4) $\widehat{\mathbb{A}}_{K} \in \mathbb{R}^{\left|\mathcal{E}_{K}\right| \times\left|\mathcal{E}_{K}\right|}$ are the symmetric and positive definite "element matrices" of the given method for each $K \in \mathcal{T}_{H}$; 5) on each $K \in \mathcal{T}_{H}$, there exists a lifting of the face normal fluxes $\mathrm{U}_{K}^{\text {ext }}=\left\{(\mathrm{U})_{\sigma}\right\}_{\sigma \in \mathcal{E}_{K}}$ to the interior of $K$ according to [16, Theorem 5.1]; 6) for $\sigma, \sigma^{\prime} \in \mathcal{E}_{H}, \mathbb{A}_{\sigma, \sigma^{\prime}}=0$ if $\sigma$ and $\sigma^{\prime}$ are not faces of the same element $K \in \mathcal{T}_{H}$ and $\mathbb{A}_{\sigma, \sigma^{\prime}}=\sum_{K \in \mathcal{T}_{H},\left\{\sigma, \sigma^{\prime}\right\} \in \mathcal{E}_{K}} \mathbf{n}_{K, \sigma} \cdot \mathbf{n}_{\sigma} \mathbf{n}_{K, \sigma^{\prime}} \cdot \mathbf{n}_{\sigma^{\prime}}\left(\widehat{\mathbb{A}}_{K}\right)_{\sigma, \sigma^{\prime}}$ otherwise.

\subsection{A fictitious flux reconstruction under Assumption 3.1}

Following the concept of lifting operators used in, e.g., Eymard et al. [41, Section 1.2], Kuznetsov and Repin [52], Brezzi et al. [16, Theorem 5.1], Beirão da Veiga [7, Section 2.1], Beirão da Veiga and Manzini [10, Section 2.4], Kuznetsov [53], Vohralík [64, Section 3.2], or Sboui et al. [61], we will now extend the fluxes $(\mathrm{U})_{\sigma}, \sigma \in \mathcal{E}_{K}$, to the interior of each polytopal element $K \in \mathcal{T}_{H}$. The obtained flux $\left.\mathbf{u}_{h}\right|_{K}$ will be the approximation for which we will estimate the error. Actually, it needs not be constructed in practice, unless one wants to compute the error in model cases with known exact flux $\mathbf{u}$; for this reason, we call it fictitious. We proceed here under the very general Assumption 3.1, the case of Assumption 3.2 is treated in Section 3.4 below.

Following [67, Theorems 7.2 and 7.3], consider the mixed finite element method on the simplicial mesh $\mathcal{T}_{K}$ to approximate separately in each element $K \in \mathcal{T}_{H}$ the problem: find $p_{K}: K \rightarrow \mathbb{R}$ such that

$$
\begin{aligned}
-\nabla \cdot\left(\underline{\mathbf{K}} \nabla p_{K}\right) & =\left.f\right|_{K}, \\
\frac{\left(p_{K}, 1\right)_{K}}{|K|} & =(\mathrm{P})_{K}, \\
-\underline{\mathbf{K}} \nabla p_{K} \cdot \mathbf{n}_{\sigma^{\prime}} & =\frac{(\mathbf{U})_{\sigma}}{|\sigma|} \quad \forall \sigma^{\prime} \in \mathcal{E}_{K, h}^{\mathrm{ext}}, \sigma^{\prime} \subset \sigma, \sigma \in \mathcal{E}_{K} .
\end{aligned}
$$

Note that if the faces of the simplicial mesh $\mathcal{T}_{h}$ do not subdivide the faces of the polytopal mesh $\mathcal{T}_{H}$, then $\mathcal{E}_{K, h}^{\text {ext }}=\mathcal{E}_{K}$, so that it is enough to consider directly all faces $\sigma \in \mathcal{E}_{K}$ in (3.6c). Henceforth, we will also use the notation

$$
(\mathrm{U})_{\sigma^{\prime}}:=\frac{(\mathrm{U})_{\sigma}}{|\sigma|}\left|\sigma^{\prime}\right| \quad \forall \sigma^{\prime} \in \mathcal{E}_{K, h}^{\mathrm{ext}}, \sigma^{\prime} \subset \sigma, \sigma \in \mathcal{E}_{K}
$$


for the normal flux over the given simplicial subface $\sigma^{\prime}$ of each original polytopal face $\sigma$.

The approximation will be done by means of the lowest-order Raviart-Thomas-Nédélec spaces over the simplicial submesh $\mathcal{T}_{K}$ of $K$ : for each polytope $K \in \mathcal{T}_{H}$, define $\mathbf{R T N}_{0}(K):=\left\{\mathbf{v}_{h} \in \mathbf{H}(\operatorname{div}, K)\right.$; $\left.\left.\mathbf{v}_{h}\right|_{\kappa} \in\left[\mathbb{P}_{0}(\kappa)\right]^{d}+\mathbf{x} \mathbb{P}_{0}(\kappa) \forall \kappa \in \mathcal{T}_{K}\right\}$. Here $\mathbb{P}_{0}(\kappa)$ stands for constants on the simplex $\kappa$, and we will also denote by $\mathbb{P}_{0}\left(\mathcal{T}_{K}\right)$ the space of piecewise constants on the polytope $K$ with respect to its mesh $\mathcal{T}_{K}$. We then set

$$
\begin{aligned}
& \mathbf{V}_{h, \mathrm{~N}}^{K}:=\left\{\mathbf{v}_{h} \in \mathbf{R T N}_{0}(K),\left\langle\mathbf{v}_{h} \cdot \mathbf{n}_{\sigma}, 1\right\rangle_{\sigma}=(\mathrm{U})_{\sigma} \forall \sigma \in \mathcal{E}_{K, h}^{\mathrm{ext}}\right\}, \\
& \mathbf{V}_{h, 0}^{K}:=\left\{\mathbf{v}_{h} \in \mathbf{R T N}_{0}(K),\left\langle\mathbf{v}_{h} \cdot \mathbf{n}_{\sigma}, 1\right\rangle_{\sigma}=0 \forall \sigma \in \mathcal{E}_{K, h}^{\mathrm{ext}}\right\}, \\
& Q_{h, \mathrm{~N}}^{K}:=\left\{q_{h} \in \mathbb{P}_{0}\left(\mathcal{T}_{K}\right) ; \frac{\left(q_{h}, 1\right)_{K}}{|K|}=(\mathrm{P})_{K}\right\}, \\
& Q_{h, 0}^{K}:=\left\{q_{h} \in \mathbb{P}_{0}\left(\mathcal{T}_{K}\right) ;\left(q_{h}, 1\right)_{K}=0\right\}
\end{aligned}
$$

and can consider:

Definition 3.3 (Element fictitious flux reconstruction). For all $K \in \mathcal{T}_{H}$, define

$$
\left.\mathbf{u}_{h}\right|_{K}:=\arg \operatorname{vin}_{\mathbf{v}_{h} \in \mathbf{V}_{h, \mathrm{~N}}^{K}, \nabla \cdot \mathbf{v}_{h}=\text { constant }}\left\|\mathbf{v}_{h}\right\|_{\underline{\mathbf{K}}^{-\frac{1}{2}} ; L^{2}(K)},
$$

i.e., $\left.\mathbf{u}_{h}\right|_{K} \in \mathbf{V}_{h, \mathrm{~N}}^{K}$ together with $\left.p_{h}\right|_{K} \in Q_{h, \mathrm{~N}}^{K}$ are given by

$$
\begin{aligned}
\left(\underline{\mathbf{K}}^{-1} \mathbf{u}_{h}, \mathbf{v}_{h}\right)_{K}-\left(p_{h}, \nabla \cdot \mathbf{v}_{h}\right)_{K}=0 & \forall \mathbf{v}_{h} \in \mathbf{V}_{h, 0}^{K}, \\
-\left(\nabla \cdot \mathbf{u}_{h}, q_{h}\right)_{K}=0 & \forall q_{h} \in Q_{h, 0}^{K} .
\end{aligned}
$$

It is worth noting that the constraint in (3.9) together with the assumption that $f$ is constant on each $K \in \mathcal{T}_{H}$, the definition of $\mathbf{V}_{h, \mathrm{~N}}^{K}$, and the flux balance (3.4) gives $\nabla \cdot \mathbf{v}_{h}=f$. It can alternatively be written as $\Pi_{Q_{h, 0}^{K}}\left(\nabla \cdot \mathbf{v}_{h}\right)=0$, which leads to (3.10b). Note also that since the normal fluxes $(\mathrm{U})_{\sigma}$ are univalued on the faces from $\mathcal{E}_{H, h}$, the resulting flux reconstruction $\mathbf{u}_{h}$ has the normal trace continuous and thus belongs to the Raviart-Thomas-Nédélec space $\mathbf{R T N}_{0}(\Omega)$ associated with the simplicial mesh $\mathcal{T}_{h}$ of the entire domain $\Omega$. In matrix form, Definition 3.3 takes the following form:

Remark 3.4 (Matrix form of problem (3.10)). Let $\mathbf{v}_{\sigma}, \sigma \in \mathcal{E}_{K, h}^{\text {int }}$, be the basis functions of the space $\mathbf{V}_{h, 0}^{K}$, and $\mathbf{v}_{\sigma}, \sigma \in \mathcal{E}_{K, h}^{\mathrm{ext}}$, be the remaining basis functions of the space $\mathbf{R T N}_{0}(K)$. Similarly, let $q_{i}, 1 \leq i \leq\left|Q_{h, 0}^{K}\right|$, be the basis functions of the space $Q_{h, 0}^{K}$. Consider these basis functions of $\mathbf{V}_{h, 0}^{K}$ and $Q_{h, 0}^{K}$ as test functions in (3.10) and develop $\left.\mathbf{u}_{h}\right|_{K}=\sum_{\sigma \in \mathcal{E}_{K, h}^{\text {ext }}}\left(\mathrm{U}_{K}^{\text {ext }}\right)_{\sigma} \mathbf{v}_{\sigma}+\sum_{\sigma \in \mathcal{E}_{K, h}^{\text {int }}}\left(\mathrm{U}_{K}^{\text {int }}\right)_{\sigma} \mathbf{v}_{\sigma}$ and $\left.p_{h}\right|_{K}=(\mathrm{P})_{K}+\sum_{i=1}^{\left|Q_{h, 0}^{K}\right|}\left(\mathrm{P}_{K}^{0}\right)_{i} q_{i}$, where $\mathrm{U}_{K}^{\mathrm{ext}}:=\left\{(\mathrm{U})_{\sigma}\right\}_{\sigma \in \mathcal{E}_{K, h}^{\mathrm{ext}}}$ and $(\mathrm{P})_{K}$ are the given data of the problem (3.6). Then (3.10) in matrix form corresponds to: find $\cup_{K}^{\mathrm{int}} \in \mathbb{R}^{\left|\mathbf{V}_{h, 0}^{K}\right|}$ and $\mathrm{P}_{K}^{0} \in \mathbb{R}^{\left|Q_{h, 0}^{K}\right|}$ such that

$$
\left(\begin{array}{cc}
\mathbb{A}_{K}^{\text {int,int }} & \left(\mathbb{B}_{K}^{0, \text { int }}\right)^{\mathrm{t}} \\
\mathbb{B}_{K}^{0, \text { int }} & 0
\end{array}\right)\left(\begin{array}{l}
\mathrm{U}_{K}^{\text {int }} \\
\mathrm{P}_{K}^{0}
\end{array}\right)=\left(\begin{array}{l}
-\mathbb{A}_{K}^{\text {int,ext }} U_{K}^{\text {ext }} \\
-\mathbb{B}_{K}^{0 \text { eext }} \bigcup_{K}^{\text {ext }}
\end{array}\right)
$$

where

$$
\begin{aligned}
\left(\mathbb{A}_{K}^{\text {int,int }}\right)_{\sigma, \sigma^{\prime}} & :=\left(\underline{\mathbf{K}}^{-1} \mathbf{v}_{\sigma^{\prime}}, \mathbf{v}_{\sigma}\right)_{K} \quad \sigma, \sigma^{\prime} \in \mathcal{E}_{K, h}^{\text {int }}, \\
\left(\mathbb{A}_{K}^{\text {int,ext }}\right)_{\sigma, \sigma^{\prime}}: & :=\left(\underline{\mathbf{K}}^{-1} \mathbf{v}_{\sigma^{\prime}}, \mathbf{v}_{\sigma}\right)_{K} \quad \sigma \in \mathcal{E}_{K, h}^{\text {int }}, \sigma^{\prime} \in \mathcal{E}_{K, h}^{\text {ext }}, \\
\left(\mathbb{B}_{K}^{0, \text { int }}\right)_{K, \sigma} & :=-\mathbf{n}_{K, \sigma} \mathbf{n}_{\sigma} \quad \sigma \in \mathcal{E}_{K, h}^{\text {int }}, \\
\left(\mathbb{B}_{K}^{0, \text { ext }}\right)_{K, \sigma} & :=-\mathbf{n}_{K, \sigma} \mathbf{n}_{\sigma} \quad \sigma \in \mathcal{E}_{K, h}^{\text {ext }} .
\end{aligned}
$$

Definition 3.3 lifts the information from the boundary of an element $K \in \mathcal{T}_{H}$ given by the fluxes $\cup_{K}^{\text {ext }}$ to the interior of the element $K$. It is thus clear that the energy $\left\|\mathbf{u}_{h}\right\|_{\underline{\mathbf{K}}^{-\frac{1}{2} ; L^{2}(K)}}$ needs to only depend on $\mathrm{U}_{K}^{\text {ext }}$. It turns out that this link can be expressed by the element matrix $\widehat{\mathbb{A}}_{\mathrm{MFE}, K}$. Define, in addition to Remark 3.4,

$$
\left(\mathbb{A}_{K}^{\mathrm{ext}, \mathrm{ext}}\right)_{\sigma, \sigma^{\prime}}:=\left(\underline{\mathbf{K}}^{-1} \mathbf{v}_{\sigma^{\prime}}, \mathbf{v}_{\sigma}\right)_{K} \quad \sigma, \sigma^{\prime} \in \mathcal{E}_{K, h}^{\mathrm{ext}} .
$$

As in [67, proof of Theorem 7.3], we indeed have: 
Lemma 3.5 (Energy norm and MFE element matrix). For each polytopal element $K \in \mathcal{T}_{H}$, define the element matrix of the mixed finite element method

$$
\widehat{\mathbb{A}}_{\mathrm{MFE}, K}:=\mathbb{A}_{K}^{\text {ext,ext }}-\left(\begin{array}{l}
\mathbb{A}_{K}^{\mathrm{int}, \text { ext }} \\
\mathbb{B}_{K}^{0, \text { ext }}
\end{array}\right)^{\mathrm{t}}\left(\begin{array}{cc}
\mathbb{A}_{K}^{\mathrm{int}, \text { int }} & \left(\mathbb{B}_{K}^{0, \text { int }}\right)^{\mathrm{t}} \\
\mathbb{B}_{K}^{0, \text { int }} & 0
\end{array}\right)^{-1}\left(\begin{array}{l}
\mathbb{A}_{K}^{\mathrm{int}, \text { ext }} \\
\mathbb{B}_{K}^{0, \text { ext }}
\end{array}\right) .
$$

Let $\left.\mathbf{u}_{h}\right|_{K} \in \mathbf{V}_{h, \mathrm{~N}}^{K}$ be given by Definition 3.3. Then

$$
\left\|\mathbf{u}_{h}\right\|_{\underline{\mathbf{K}}^{-\frac{1}{2} ; L^{2}(K)}}^{2}=\left(\mathrm{U}_{K}^{\mathrm{ext}}\right)^{\mathrm{t}} \widehat{\mathbb{A}}_{\mathrm{MFE}, K} \mathrm{U}_{K}^{\mathrm{ext}} .
$$

Proof. Let $K \in \mathcal{T}_{H}$. Following Remark 3.4, decompose $\left.\mathbf{u}_{h}\right|_{K}=\mathbf{u}_{h, K}^{\text {ext }}+\mathbf{u}_{h, K}^{\text {int }}$ and $\left.p_{h}\right|_{K}=(\mathrm{P})_{K}+p_{h, K}^{0}$. Note that choosing $\mathbf{u}_{h, K}^{\text {int }} \in \mathbf{V}_{h, 0}^{K}$ as the test function in (3.10a) and $p_{h, K}^{0} \in Q_{h, 0}^{K}$ as the test function in (3.10b), one has

$$
\left(\underline{\mathbf{K}}^{-1} \mathbf{u}_{h}, \mathbf{u}_{h, K}^{\mathrm{int}}\right)_{K}=\left(p_{h, K}^{0}, \nabla \cdot \mathbf{u}_{h, K}^{\mathrm{int}}\right)_{K}=-\left(p_{h, K}^{0}, \nabla \cdot \mathbf{u}_{h, K}^{\mathrm{ext}}\right)_{K},
$$

where we have used $\left((\mathrm{P})_{K}, \nabla \cdot \mathbf{u}_{h, K}^{\text {int }}\right)_{K}=0$. Consequently,

$$
\begin{aligned}
\left\|\mathbf{u}_{h}\right\|_{\underline{\mathbf{K}}^{-\frac{1}{2}} ; L^{2}(K)}^{2} & =\left(\underline{\mathbf{K}}^{-1} \mathbf{u}_{h}, \mathbf{u}_{h}\right)_{K} \\
& =\left(\underline{\mathbf{K}}^{-1} \mathbf{u}_{h}, \mathbf{u}_{h, K}^{\text {int }}\right)_{K}+\left(\underline{\mathbf{K}}^{-1} \mathbf{u}_{h, K}^{\text {int }}, \mathbf{u}_{h, K}^{\text {ext }}\right)_{K}+\left(\underline{\mathbf{K}}^{-1} \mathbf{u}_{h, K}^{\text {ext }}, \mathbf{u}_{h, K}^{\text {ext }}\right)_{K} \\
& =-\left(p_{h, K}^{0}, \nabla \cdot \mathbf{u}_{h, K}^{\text {ext }}\right)_{K}+\left(\underline{\mathbf{K}}^{-1} \mathbf{u}_{h, K}^{\text {int }}, \mathbf{u}_{h, K}^{\text {ext }}\right)_{K}+\left(\underline{\mathbf{K}}^{-1} \mathbf{u}_{h, K}^{\text {ext }}, \mathbf{u}_{h, K}^{\text {ext }}\right)_{K} \\
& =\left(\mathbf{U}_{K}^{\text {ext }}\right)^{\mathrm{t}}\left(\begin{array}{l}
\mathbb{A}_{K}^{\text {int,ext }} \\
\mathbb{B}_{K}^{0, \text {ext}}
\end{array}\right)^{\mathrm{t}}\left(\begin{array}{c}
\mathbf{U}_{K}^{\text {int }} \\
P_{K}^{0}
\end{array}\right)+\left(\mathbf{U}_{K}^{\text {ext }}\right)^{\mathrm{t}} \mathbb{A}_{K}^{\text {ext }, \text { ext }} U_{K}^{\text {ext }} .
\end{aligned}
$$

Combining this with the MFE matrix form (3.11) and the MFE element matrix definition (3.12) finishes the proof.

\subsection{A fictitious flux reconstruction under Assumption 3.2}

There exist other ways how to lift the face normal fluxes $\bigcup_{K}^{\text {ext }}$ into the interior of each polytope $K \in \mathcal{T}_{H}$ than via Definition 3.3. In particular, under Assumption 3.2, one can use the general notion of the lifting operator from mimetic finite differences according to [16, Theorem 5.1] to define $\tilde{\mathbf{u}}_{h} \in \mathbf{H}(\operatorname{div}, K)$. This lifting is truly fictitious in the sense that it cannot in general be constructed in practice and leads to:

Lemma 3.6 (Energy norm and given discretization scheme element matrix). Let a discretization scheme of the form of Assumption 3.2 be given, leading to the element matrices $\widehat{\mathbb{A}}_{K}$ and normal face fluxes $\cup_{K}^{\text {ext }}=$ $\left\{(\mathrm{U})_{\sigma}\right\}_{\sigma \in \mathcal{E}_{K}}$ for all $K \in \mathcal{T}_{H}$. Let $\mathcal{E}_{K, h}^{\mathrm{ext}}=\mathcal{E}_{K}$, i.e., the simplicial faces of $\mathcal{T}_{h}$ do not subdivide the faces of the polytopal mesh $\mathcal{T}_{H}$. Let finally $\tilde{\mathbf{u}}_{h} \in \mathbf{H}(\operatorname{div}, K)$ be the lifting according to [16, Theorem 5.1]. Then

$$
\left\|\tilde{\mathbf{u}}_{h}\right\|_{\underline{\mathbf{K}}^{-\frac{1}{2}} ; L^{2}(K)}^{2}=\left(\mathrm{U}_{K}^{\mathrm{ext}}\right)^{\mathrm{t}} \widehat{\mathbb{A}}_{K} \mathrm{U}_{K}^{\mathrm{ext}} .
$$

Proof. Property (3.13) is nothing but equality (5.7) from [16, Theorem 5.1].

To interconnect Lemmas 3.5 and 3.6, we now evoke [67, Theorem 7.3]. This shows that the mixed finite element element matrix $\widehat{\mathbb{A}}_{\mathrm{MFE}, K}$ defined by (3.12) belongs to the same family as the element matrices $\widehat{\mathbb{A}}_{K}$ of mimetic finite differences, mixed finite volumes, and hybrid finite volumes of Assumption 3.2 when the simplicial faces of $\mathcal{T}_{h}$ do not subdivide the faces of the polytopal mesh $\mathcal{T}_{H}$. This leads to the following result:

Lemma 3.7 (Approximate energy norm). Let the assumptions of Lemmas 3.5 and 3.6 be satisfied. Then there exist two constants $c, C>0$ independent of the matrices $\widehat{\mathbb{A}}_{K}, \widehat{\mathbb{A}}_{\mathrm{MFE}, K}$, and the mesh size but depending on the shape-regularity of the simplicial submesh $\mathcal{T}_{K}$ such that

$$
c\left(\mathrm{U}_{K}^{\text {ext }}\right)^{\mathrm{t}} \widehat{\mathbb{A}}_{K} \mathrm{U}_{K}^{\text {ext }} \leq\left\|\mathbf{u}_{h}\right\|_{\underline{\mathbf{K}}^{-\frac{1}{2}} ; L^{2}(K)}^{2} \leq C\left(\mathrm{U}_{K}^{\text {ext }}\right)^{\mathrm{t}} \widehat{\mathbb{A}}_{K} \mathrm{U}_{K}^{\text {ext }}
$$

that we denote by

$$
\left\|\mathbf{u}_{h}\right\|_{\underline{\mathbf{K}}^{-\frac{1}{2}} ; L^{2}(K)}^{2} \approx\left(\mathrm{U}_{K}^{\mathrm{ext}}\right)^{\mathrm{t}} \widehat{\mathbb{A}}_{K} \mathrm{U}_{K}^{\mathrm{ext}}
$$


Proof. Any scheme of the form of Assumption 3.2 with the lifting operator according to [16, Theorem 5.1] satisfies inequalities (5.8) from [16, Theorem 5.1]. As mixed finite elements on polytopal meshes belong to the mimetic finite difference family by [67, Theorem 7.3], see also Lemma 3.5, this implies (3.14).

\subsection{A fictitious potential reconstruction under Assumption 3.1}

As a second ingredient of our a posteriori analysis, we will now define a simple potential reconstruction $s_{h} \in \mathbb{P}_{1}\left(\mathcal{T}_{h}\right) \cap H_{0}^{1}(\Omega)$, piecewise affine with respect to the simplicial submesh $\mathcal{T}_{h}$ and $H_{0}^{1}(\Omega)$-conforming. Crucially, it will also be fictitious in the sense that it does not need to be constructed in practice in order to evaluate the resulting a posteriori error estimate, where we will go along with its point values.

In the spirit of usual averaging operators, cf. Durán and Padra [36], Achdou et al. [3], Karakashian and Pascal [49], Ainsworth [4], or Burman and Ern [19], the easiest way is to set the nodal values $s_{h}(\mathbf{a})$ in the vector $\left\{(\mathrm{S})_{\mathbf{a}}\right\}_{\mathbf{a} \in \mathcal{V}_{h}}$ by average values of $(\mathrm{P})_{K}$ in the neighboring elements:

Definition 3.8 (Element potential reconstruction). For each polytopal element $K \in \mathcal{T}_{H}$ and each vertex $\mathbf{a}$ of the simplicial mesh $\mathcal{T}_{h}$ lying on $\partial K$ but not on $\partial \Omega$, let $\mathcal{T}_{\mathbf{a}}$ denote the set of polytopal elements $K \in \mathcal{T}_{H}$ sharing a. We set

$$
(\mathrm{S})_{\mathbf{a}}:=\left(\mathrm{S}_{K}\right)_{\mathbf{a}}:=\frac{1}{\left|\mathcal{T}_{\mathbf{a}}\right|} \sum_{K \in \mathcal{T}_{\mathbf{a}}}(\mathrm{P})_{K}
$$

We also set $(\mathrm{S})_{\mathbf{a}}:=\left(\mathrm{S}_{K}\right)_{\mathbf{a}}:=(\mathrm{P})_{K}$ for the vertex $\mathbf{a}$ of $\mathcal{T}_{h}$ lying inside $K \in \mathcal{T}_{H}$ and $(\mathrm{S})_{\mathbf{a}}:=\left(\mathrm{S}_{K}\right)_{\mathbf{a}}:=0$ for any vertex a lying simultaneously on $\partial K$ and the domain boundary $\partial \Omega$, in accordance with the boundary condition (3.1b).

For each polytopal cell $K \in \mathcal{T}_{H}$, let $\mathrm{S}_{K} \in \mathbb{R}^{\left|\mathcal{V}_{K, h}\right|}$ collect the values $\left(\mathrm{S}_{K}\right)_{\mathbf{a}}$ for the vertices a of the simplicial mesh $\mathcal{T}_{K}$ lying on the boundary $\partial K$ and the value inside $K$, see Figure 2 in two space dimensions. We will also need the vector $S_{K}^{\text {ext }} \in \mathbb{R}^{\left|\mathcal{E}_{K, h}^{\text {ext }}\right|}$ collecting the values associated with faces $\sigma \in \mathcal{E}_{K, h}^{\text {ext }}$ that we prescribe by

$$
\left(\mathrm{S}_{K}^{\mathrm{ext}}\right)_{\sigma}:=\frac{1}{d} \sum_{\mathbf{a} \in \mathcal{V}_{\sigma}}(\mathrm{S})_{\mathbf{a}}
$$

where, recall, $\mathcal{V}_{\sigma}$ collects the vertices of the given face $\sigma \in \mathcal{E}_{K, h}^{\mathrm{ext}}$. Note that $\left(\mathrm{S}_{K}^{\mathrm{ext}}\right)_{\sigma}=s_{h}\left(\mathbf{x}_{\sigma}\right)$, i.e., it is the punctual value of the reconstruction $s_{h}$ in the face barycenter $\mathbf{x}_{\sigma}, \sigma \in \mathcal{E}_{K, h}^{\text {ext }}$. Figure 2 also gives an illustration of the vector $\mathrm{S}_{K}^{\mathrm{ext}}$.

Consider the usual hat basis functions $\psi_{\mathbf{a}}$ on the simplicial mesh $\mathcal{T}_{h}: \psi_{\mathbf{a}}$ is piecewise affine on $\mathcal{T}_{h}$ and $H_{0}^{1}(\Omega)$-conforming, $\psi_{\mathbf{a}}\left(\mathbf{a}^{\prime}\right)=1$ if $\mathbf{a}=\mathbf{a}^{\prime}$ and 0 otherwise, where $\mathbf{a}, \mathbf{a}^{\prime} \in \mathcal{V}_{h}$ are the vertices of $\mathcal{T}_{h}$. We will need below the stiffness matrix $\widehat{\mathbb{S}}_{\mathrm{FE}, K} \in \mathbb{R}^{\left|\mathcal{V}_{K, h}\right| \times\left|\mathcal{V}_{K, h}\right|}$ of a polytopal element $K \in \mathcal{T}_{H}$ defined by

$$
\left(\widehat{\mathbb{S}}_{\mathrm{FE}, K}\right)_{\mathbf{a}, \mathbf{a}^{\prime}}:=\left(\underline{\mathbf{K}} \nabla \psi_{\mathbf{a}^{\prime}}, \nabla \psi_{\mathbf{a}}\right)_{K} \quad \mathbf{a}, \mathbf{a}^{\prime} \in \mathcal{V}_{K, h} .
$$

An immediate consequence is that

$$
\left\|\underline{\mathbf{K}} \nabla s_{h}\right\|_{\underline{\mathbf{K}}^{-\frac{1}{2}} ; L^{2}(K)}^{2}=\mathrm{S}_{K}^{\mathrm{t}} \widehat{\mathbb{S}}_{\mathrm{FE}, K} \mathrm{~S}_{K} .
$$

Similarly, we let the element mass matrix $\widehat{\mathbb{M}}_{\mathrm{FE}, K} \in \mathbb{R}^{\left|\mathcal{V}_{K, h}\right| \times\left|\mathcal{V}_{K, h}\right|}$ be given by

$$
\left(\widehat{\mathbb{M}}_{\mathrm{FE}, K}\right)_{\mathbf{a}, \mathbf{a}^{\prime}}:=\left(\psi_{\mathbf{a}^{\prime}}, \psi_{\mathbf{a}}\right)_{K} \quad \mathbf{a}, \mathbf{a}^{\prime} \in \mathcal{V}_{K, h} .
$$

Then

$$
\left(1, s_{h}\right)_{K}=1^{\mathrm{t}} \widehat{\mathbb{M}}_{\mathrm{FE}, K} \mathrm{~S}_{K} .
$$

Remark 3.9 (Element matrices $\widehat{\mathbb{S}}_{\mathrm{FE}, K}$ and $\widehat{\mathbb{M}}_{\mathrm{FE}, K}$ ). To obtain the finite element stiffness and mass matrices $\widehat{\mathbb{S}}_{\mathrm{FE}, K}$ of (3.18) and $\widehat{\mathbb{M}}_{\mathrm{FE}, K}$ of (3.20), one could use a finite element assembly code linked to a simplicial mesh on the polytopal cell $K \in \mathcal{T}_{H}$. Note, however, that there exist simple analytical formulas for the basis functions $\psi_{\mathbf{a}}$ of affine Lagrange finite elements and for their gradients $\nabla \psi_{\mathbf{a}}$ that merely necessitate the position of the vertices of the polygonal element $K$ and of the central point. In this sense, we say that neither $\mathcal{T}_{h}$ nor $\mathcal{T}_{K}$ need not be constructed in practice in order to obtain $\widehat{\mathbb{S}}_{\mathrm{FE}, K}$ and $\widehat{\mathbb{M}}_{\mathrm{FE}, K}$. 


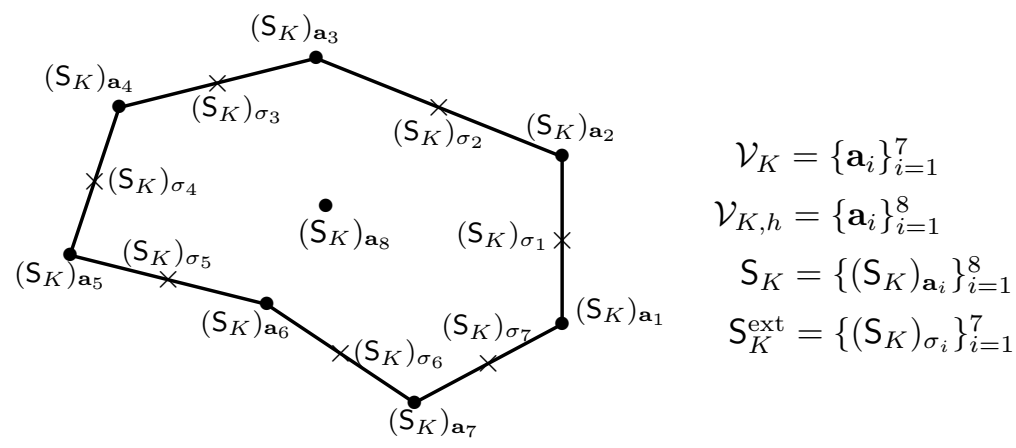

Figure 2: Example of nodal and facial potential reconstruction values $S_{K}$ and $S_{K}^{\text {ext }}$

\subsection{A fictitious potential reconstruction under Assumption 3.2}

A more straightforward and precise fictitious potential reconstruction can be designed under Assumption 3.2. The main idea is that any scheme of the form (3.5) can be hybridized, following, e.g., Roberts and Thomas [60], Brezzi and Fortin [15], or Droniou et al. [34], giving rise to one Lagrange multiplier $\Lambda_{\sigma}$ per face $\sigma \in \mathcal{E}_{H}$. Indeed, consider the line associated with a given face $\sigma \in \mathcal{E}_{H}$ in the first block equation of (3.5). For an interior face $\sigma$, let it be shared by two polytopes $K, K^{\prime} \in \mathcal{T}_{H}$ such that $\mathbf{n}_{\sigma}$ points from $K$ to $K^{\prime}$. From the structure of the matrices $\mathbb{A}$ and $\mathbb{B}$ supposed in Assumption 3.2, it follows that

$$
\sum_{\sigma^{\prime} \in \mathcal{E}_{K}} \mathbf{n}_{K, \sigma^{\prime}} \cdot \mathbf{n}_{\sigma^{\prime}}\left(\widehat{\mathbb{A}}_{K}\right)_{\sigma, \sigma^{\prime}}(\mathrm{U})_{\sigma^{\prime}}-(\mathrm{P})_{K}=\sum_{\sigma^{\prime} \in \mathcal{E}_{K^{\prime}}} \mathbf{n}_{K^{\prime}, \sigma^{\prime}} \cdot \mathbf{n}_{\sigma^{\prime}}\left(\widehat{\mathbb{A}}_{K^{\prime}}\right)_{\sigma, \sigma^{\prime}}(\mathrm{U})_{\sigma^{\prime}}-(\mathrm{P})_{K^{\prime}},
$$

so that these expressions are univalued from both elements $K$ and $K^{\prime}$. As such an expression is also clearly univalued on boundary faces, we can define the Lagrange multipliers

$$
\Lambda_{\sigma}:=(\mathrm{P})_{K}-\sum_{\sigma^{\prime} \in \mathcal{E}_{K}} \mathbf{n}_{K, \sigma^{\prime}} \cdot \mathbf{n}_{\sigma^{\prime}}\left(\widehat{\mathbb{A}}_{K}\right)_{\sigma, \sigma^{\prime}}(\mathrm{U})_{\sigma^{\prime}}
$$

for each element $K \in \mathcal{T}_{H}$ and each face $\sigma \in \mathcal{E}_{K}$. We impose the nodal values $s_{h}(\mathbf{a})$ of $s_{h}$ piecewise affine with respect to the simplicial submesh $\mathcal{T}_{h}$ and $H_{0}^{1}(\Omega)$-conforming in the vector $\left\{(\mathrm{S})_{\mathbf{a}}\right\}_{\mathbf{a} \in \mathcal{V}_{h}}$ by averaging the values $\Lambda_{\sigma}$ :

Definition 3.10 (Element potential reconstruction based on Lagrange multipliers). For each polytopal element $K \in \mathcal{T}_{H}$ and each vertex a of the simplicial mesh $\mathcal{T}_{K}$ lying on $\partial K$ but not on the boundary $\partial \Omega$ and shared by faces of the polytope $K$ from $\mathcal{E}_{K}$ collected in the set $\mathcal{E}_{K, \mathbf{a}}$, define first $\left(\overline{\mathrm{S}}_{\mathbf{a}}:=\left(\overline{\mathrm{S}}_{K}\right)_{\mathbf{a}}:=\right.$ $\frac{1}{\left|\mathcal{E}_{K, \mathbf{a}}\right|} \sum_{\sigma \in \mathcal{E}_{K, \mathbf{a}}} \Lambda_{\sigma}$. On the boundary $\partial \Omega$, define $(\overline{\mathbf{S}})_{\mathbf{a}}:=\left(\overline{\mathrm{S}}_{K}\right)_{\mathbf{a}}:=0$. Let now a vertex a of the simplicial mesh $\mathcal{T}_{h}$ lie on $\partial K$ for some $K \in \mathcal{T}_{H}$ and let $\mathcal{T}_{\mathbf{a}}$ denote the elements $K^{\prime} \in \mathcal{T}_{H}$ sharing $\mathbf{a}$. We then set

$$
(\mathrm{S})_{\mathbf{a}}:=\left(\mathrm{S}_{K}\right)_{\mathbf{a}}:=\frac{1}{\left|\mathcal{T}_{\mathbf{a}}\right|} \sum_{K^{\prime} \in \mathcal{T}_{\mathbf{a}}}\left(\overline{\mathrm{S}}_{K^{\prime}}\right)_{\mathbf{a}}
$$

together with $\left(\mathrm{S}_{K}\right)_{\mathbf{a}}:=(\mathrm{P})_{K}$ for the vertex a of $\mathcal{T}_{h}$ lying inside $K \in \mathcal{T}_{H}$.

We finish this section by a remark on an alternative, still more precise potential reconstruction, that we will namely use for comparison in numerical experiments below:

Remark 3.11 (Piecewise quadratic potential reconstruction). Let $\left(\mathbf{u}_{h}, p_{h}\right)$ be given by Definition 3.3. Following [63, Section 4.1], let $\tilde{p}_{h}$ be a piecewise quadratic polynomial on the simplicial mesh $\mathcal{T}_{h}$ given by

$$
-\left.\underline{\mathbf{K}} \nabla \tilde{p}_{h}\right|_{\kappa}=\left.\mathbf{u}_{h}\right|_{\kappa}, \quad \frac{\left(\tilde{p}_{h}, 1\right)_{\kappa}}{|\kappa|}=\left.p_{h}\right|_{\kappa} \quad \forall \kappa \in \mathcal{T}_{h}
$$

A typically more precise piecewise quadratic but computationally more demanding potential reconstruction $s_{h} \in \mathbb{P}_{2}\left(\mathcal{T}_{h}\right) \cap H_{0}^{1}(\Omega)$ can be obtained by averaging the values of $\tilde{p}_{h}$ in all Lagrangian degrees of freedom, cf. [63, Section 4.2]. 


\subsection{A simple guaranteed a posteriori error estimate}

We now present our simple a posteriori error estimate for the model problem (3.1). Our main result is given for the error between the exact Darcy velocity $\mathbf{u} \in \mathbf{H}(\operatorname{div}, \Omega)$ of $(3.2)-(3.3)$ and the reconstruction $\mathbf{u}_{h} \in \mathbf{R T N}_{0}(\Omega)$ of Definition (3.3), under the general Assumption 3.1:

Theorem 3.12 (A simple guaranteed a posteriori error estimate). Let $\mathbf{u}$ be given by (3.2)-(3.3). For any polytopal discretization satisfying Assumption 3.1, let the flux $\mathbf{u}_{h} \in \mathbf{R} \mathbf{T N}_{0}(\Omega)$ be reconstructed following Definition 3.3 with the vectors $\bigcup_{K}^{\text {ext }}$ given by (3.7). Let the element matrices $\widehat{\mathbb{A}}_{\mathrm{MFE}, K}, \widehat{\mathbb{S}}_{\mathrm{FE}, K}$, and $\widehat{\mathbb{M}}_{\mathrm{FE}, K}$ be respectively defined by (3.12), (3.18), and (3.20). Let finally the vectors $\mathrm{S}_{K}$ and $\mathrm{S}_{K}^{\text {ext }}$ be given by (3.16), (3.17), or alternatively, for schemes of the form of Assumption 3.2, by (3.22), (3.17). Then there holds

$$
\left\|\mathbf{u}-\mathbf{u}_{h}\right\|_{\underline{\mathbf{K}}^{-\frac{1}{2} ; L^{2}(\Omega)}} \leq\left\{\sum_{K \in \mathcal{T}_{H}} \eta_{K}^{2}\right\}^{\frac{1}{2}}
$$

where

$$
\eta_{K}^{2}:=\left(\mathrm{U}_{K}^{\mathrm{ext}}\right)^{\mathrm{t}} \widehat{\mathbb{A}}_{\mathrm{MFE}, K} \mathrm{U}_{K}^{\mathrm{ext}}+\mathrm{S}_{K}^{\mathrm{t}} \widehat{\mathbb{S}}_{\mathrm{FE}, K} \mathrm{~S}_{K}+2\left(\mathrm{U}_{K}^{\mathrm{ext}}\right)^{\mathrm{t}} \mathrm{S}_{K}^{\mathrm{ext}}-2(\mathrm{~F})_{K}|K|^{-1} 1^{\mathrm{t}} \widehat{\mathbb{M}}_{\mathrm{FE}, K} \mathrm{~S}_{K}
$$

Proof. The assumption that the source term $f$ is elementwise constant and (3.4) of Assumption 3.1 together with Definition 3.3 imply $\nabla \cdot \mathbf{u}_{h}=f$. Thus, as $\mathbf{u}_{h} \in \mathbf{H}(\operatorname{div}, \Omega)$, an argument of the type of the Prager-Synge equality [58] gives

$$
\left\|\mathbf{u}-\mathbf{u}_{h}\right\|_{\underline{\mathbf{K}}^{-\frac{1}{2} ; L^{2}(\Omega)}}=\inf _{v \in H_{0}^{1}(\Omega)}\left\|\mathbf{u}_{h}+\underline{\mathbf{K}} \nabla v\right\|_{\underline{\mathbf{K}}^{-\frac{1}{2} ; L^{2}(\Omega)}},
$$

see, e.g., [65, Theorem 6.1]. Consequently, for an arbitrary $s_{h} \in H_{0}^{1}(\Omega)$,

$$
\left\|\mathbf{u}-\mathbf{u}_{h}\right\|_{\underline{\mathbf{K}}^{-\frac{1}{2}} ; L^{2}(\Omega)} \leq\left\|\mathbf{u}_{h}+\underline{\mathbf{K}} \nabla s_{h}\right\|_{\underline{\mathbf{K}}^{-\frac{1}{2}} ; L^{2}(\Omega)} .
$$

We now choose for $s_{h}$ the fictitious potential reconstruction $s_{h}$ of Definition 3.8, continuous and piecewise affine with respect to the simplicial submesh $\mathcal{T}_{h}$ and given by the nodal values of the vector S. Developing elementwise, we obtain, for each polytopal mesh element $K \in \mathcal{T}_{H}$,

$$
\left\|\mathbf{u}_{h}+\underline{\mathbf{K}} \nabla s_{h}\right\|_{\underline{\mathbf{K}}^{-\frac{1}{2} ; L^{2}(K)}}^{2}=\left\|\mathbf{u}_{h}\right\|_{\underline{\mathbf{K}}^{-\frac{1}{2} ; L^{2}(K)}}^{2}+2\left(\mathbf{u}_{h}, \nabla s_{h}\right)_{K}+\left\|\underline{\mathbf{K}} \nabla s_{h}\right\|_{\underline{\mathbf{K}}^{-\frac{1}{2} ; L^{2}(K)}}^{2} .
$$

We now use Lemma 3.5 for the first term and (3.19) for the last one. For the middle term, recall first that the normal components of vector fields in $\mathbf{R T N}_{0}(\Omega)$ are constant on each face. Thus the Green theorem together with (3.17) and (3.21) give

$$
\left(\mathbf{u}_{h}, \nabla s_{h}\right)_{K}=\left\langle\mathbf{u}_{h} \cdot \mathbf{n}, s_{h}\right\rangle_{\partial K}-\left(\nabla \cdot \mathbf{u}_{h}, s_{h}\right)_{K}=\left(\mathrm{U}_{K}^{\mathrm{ext}}\right)^{\mathrm{t}} \mathrm{S}_{K}^{\mathrm{ext}}-(\mathrm{F})_{K}|K|^{-1} 1^{\mathrm{t}} \widehat{\mathrm{M}}_{\mathrm{FE}, K} \mathrm{~S}_{K} \cdot
$$

Thus the proof is finished.

Note that the estimate of Theorem 3.12 takes a simple form of matrix-vector multiplication on each polytopal mesh element $K \in \mathcal{T}_{H}$, and yet it delivers a guaranteed upper bound on the Darcy velocity error. Under Assumption 3.2, the following result also holds true:

Corollary 3.13 (A simple guaranteed estimate with the given element matrices $\widehat{\mathbb{A}}_{K}$ ). Let u be given by (3.2)-(3.3). For any polytopal discretization of the form of Assumption 3.2, let $\widehat{\mathbb{A}}_{K}$ be its element matrices. For all $K \in \mathcal{T}_{H}$, let $\tilde{\mathbf{u}}_{h} \in \mathbf{H}(\operatorname{div}, K)$ be the lifting according to [16, Theorem 5.1], see Lemma 3.6. Let the element matrices $\widehat{\mathbb{S}}_{\mathrm{FE}, K}$ and $\widehat{\mathbb{M}}_{\mathrm{FE}, K}$ be respectively defined by (3.18) and (3.20). Let finally the vectors $\mathrm{S}_{K}$ and $\mathrm{S}_{K}^{\mathrm{ext}}$ be given by (3.22) and (3.17). Then there holds

$$
\left\|\mathbf{u}-\tilde{\mathbf{u}}_{h}\right\|_{\underline{\mathbf{K}}^{-\frac{1}{2}} ; L^{2}(\Omega)} \leq\left\{\sum_{K \in \mathcal{T}_{H}} \tilde{\eta}_{K}^{2}\right\}^{\frac{1}{2}}
$$

where

$$
\tilde{\eta}_{K}^{2}:=\left(\mathrm{U}_{K}^{\text {ext }}\right)^{\mathrm{t}} \widehat{\mathbb{A}}_{K} \mathrm{U}_{K}^{\text {ext }}+\mathrm{S}_{K}^{\mathrm{t}} \widehat{\mathbb{S}}_{\mathrm{FE}, K} \mathrm{~S}_{K}+2\left(\mathrm{U}_{K}^{\text {ext }}\right)^{\mathrm{t}} \mathrm{S}_{K}^{\text {ext }}-2(\mathrm{~F})_{K}|K|^{-1} 1^{\mathrm{t}} \widehat{\mathbb{M}}_{\mathrm{FE}, K} \mathrm{~S}_{K}
$$


Proof. The proof is similar to that of Theorem 3.12, using the facts that $\tilde{\mathbf{u}}_{h} \in \mathbf{H}(\operatorname{div}, \Omega), \nabla \cdot \tilde{\mathbf{u}}_{h}=f$, and $\tilde{\mathbf{u}}_{h} \cdot \mathbf{n}_{\sigma}=(\mathrm{U})_{\sigma} /|\sigma|$ for all $\sigma \in \mathcal{E}_{K}$ following [16, equality (5.4)], and replacing Lemma 3.5 by Lemma 3.6.

Finally, relying on Lemma 3.7, we also have:

Corollary 3.14 (A simple estimate with the given element matrices $\widehat{\mathbb{A}}_{K}$ ). Let the assumptions of Corollary 3.13 hold, let $\mathcal{E}_{K, h}^{\mathrm{ext}}=\mathcal{E}_{K}$, i.e., the simplicial faces of $\mathcal{T}_{h}$ do not subdivide the faces of the polytopal mesh $\mathcal{T}_{H}$, and let the flux $\mathbf{u}_{h} \in \mathbf{R T N}_{0}(\Omega)$ be constructed by Definition 3.3. Then

$$
\left\|\mathbf{u}-\mathbf{u}_{h}\right\|_{\underline{\mathbf{K}}^{-\frac{1}{2}} ; L^{2}(\Omega)} \lesssim\left\{\sum_{K \in \mathcal{T}_{H}} \tilde{\eta}_{K}^{2}\right\}^{\frac{1}{2}},
$$

where $\tilde{\eta}_{K}$ is given by (3.28) and the "approximately less than or equal to" denoted by $\lesssim$ stems from the approximation (3.15).

A few comments are in order:

Remark 3.15 (Comparison of Theorem 3.12 with Corollaries 3.13 and 3.14). Theorem 3.12 is applicable to any scheme of the form of Assumption 3.1 and relies on the the mixed finite element matrices $\widehat{\mathbb{A}}_{\mathrm{MFE}, K}$ that one needs to construct by (3.12). In contrast, Corollaries 3.13 and 3.14 are only applicable to schemes of the form of Assumption 3.2, but use directly the matrices $\widehat{\mathbb{A}}_{K}$. The estimates (3.23) and (3.29) hold for the reconstruction $\mathbf{u}_{h}$ that one can compute in practice if needed by Definition 3.3, whereas (3.27) only holds for the generally unavailable lifting $\tilde{\mathbf{u}}_{h}$, as in [7, 10]. Finally, both (3.23) and (3.27) are guaranteed, whereas (3.29) is not. In the numerical experiments in Section 4 below, though, it is hard to distinguish the two estimators (3.24) and (3.28).

Remark 3.16 (Simplicial submeshes and solution of local problems). We can avoid the physical construction of the simplicial submeshes $\mathcal{T}_{K}$ under the conditions that 1) the given scheme takes the form of Assumption 3.2; 2) the elemental matrices $\widehat{\mathbb{A}}_{K}$ are explicitly given; 3) the stiffness matrix $\widehat{\mathbb{S}}_{\mathrm{FE}, K}$ of (3.18) and the mass matrix $\widehat{\mathbb{M}}_{\mathrm{FE}, K}$ of (3.20) of the conforming finite element method on $\mathcal{T}_{K}$ for each polytopal cell $K \in \mathcal{T}_{H}$ are constructed from the geometry $K$ only in the sense of Remark 3.9; 4) we use the estimator $\eta_{K}$ (3.28) of Corollary 3.13 or 3.14. Note, however, that one may need a submesh of each polytopal cell $K$ already to produce the scheme element matrix $\widehat{\mathbb{A}}_{K}$. Similarly, a typical implementation of $\widehat{\mathbb{A}}_{\mathrm{MFE}, K}(3.12)$ will also need the submesh $\mathcal{T}_{K}$. Remark finally that behind (3.12), there is the local Neumann problem of Definition 3.3.

\section{Numerical experiments: single-phase Darcy flow}

The purpose of this section is to numerically illustrate the performance of the estimators of Theorem 3.12, as well as the simplified estimate (3.29) of Corollary 3.14. We also compare this methodology to a reference estimate on the simplicial submesh in particular using the potential reconstruction of Remark 3.11. The test is taken from [55], where a collection of two-dimensional elliptic problems for testing adaptive grid refinement algorithms is proposed. We approximate $-\Delta p=f$ on the space domain $\Omega=(0,1)^{2}$ where the analytical solution is $2^{4 \alpha} x^{\alpha}(1-x)^{\alpha} y^{\alpha}(1-y)^{\alpha}$ with $\alpha=200$, see Figure 3. The source term $f$ prescribed correspondingly and we neglect here that it is not piecewise constant, i.e., the data oscillation. We use homogeneous Dirichlet boundary conditions.

The mesh $\mathcal{T}_{H}$ consists of general polygonal elements, with the fictitious triangular submesh $\mathcal{T}_{h}$ shown in Figure 4. We consider the hybrid finite volume (HFV) discretization (Droniou et al. [42] or [34, Section 2.2]), taking the form (3.5) with the matrix $\mathbb{A}$ formed by local element matrices $\widehat{\mathbb{A}}_{K}$. We compare three versions of a posteriori error estimates: a) the estimators $\eta_{K}$ in (3.24) of Theorem 3.12 are replaced by the expression $\left\|\underline{\mathbf{K}}^{-\frac{1}{2}} \mathbf{u}_{h}+\underline{\mathbf{K}}^{\frac{1}{2}} \nabla s_{h}\right\|_{K}$, where $\left.\mathbf{u}_{h}\right|_{K} \in \mathbf{V}_{h, \mathrm{~N}}^{K}$ is given in Definition 3.3 and $s_{h} \in \mathbb{P}_{2}\left(\mathcal{T}_{h}\right) \cap H_{0}^{1}(\Omega)$ is described in Remark 3.11 (called triangular MFE estimate); b) Theorem 3.12 is used, with the estimators $\eta_{K}$ evaluated via the matrix form (3.24), relying on the element matrices $\widehat{\mathbb{A}}_{\mathrm{MFE}, K}, \widehat{\mathbb{S}}_{\mathrm{FE}, K}$, and $\widehat{\mathbb{M}}_{\mathrm{FE}, K}$ of respectively (3.12), 

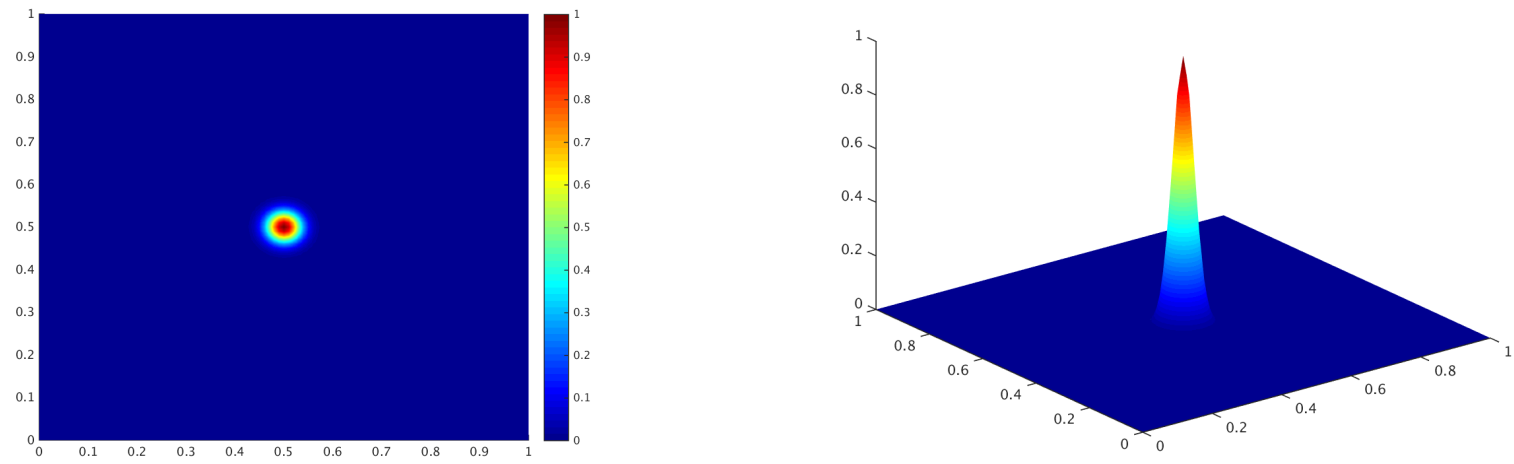

Figure 3: The solution of the analytic problem with $\alpha=200$

(3.18), and (3.20) (called polygonal MFE estimate); c) Corollary 3.14 is employed, with the bound (3.29) and the estimators of (3.28) using the element matrix $\widehat{\mathbb{A}}_{K}$ of the HFV scheme that is already available (called polygonal HFV estimate). In the last two cases, the vectors $S_{K}$ and $S_{K}^{\text {ext }}$ are given by (3.16) and (3.17).
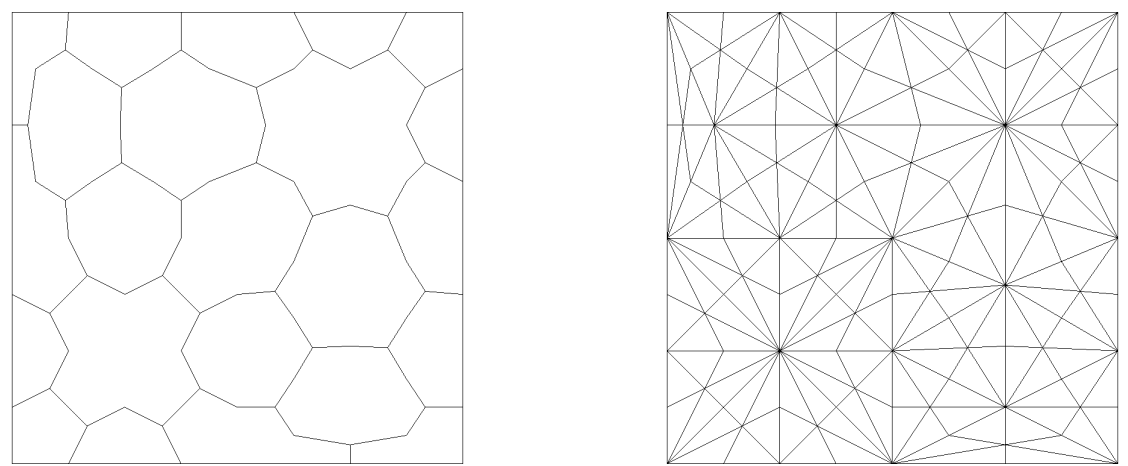

Figure 4: An example of a polygonal mesh $\mathcal{T}_{H}$ and the corresponding triangular submesh $\mathcal{T}_{h}$

Note that if the mixed finite element method on polygonal meshes of [67, Theorem 7.2] was used instead of the HFV discretization, the first procedure would be, following [67, Remark 7.3], fully equivalent to solving the problem (3.1) directly on the simplicial mesh $\mathcal{T}_{h}$ by the lowest-order Raviart-Thomas-Nédélec mixed finite element method and applying the a posteriori error estimates of [63]. This is why the triangular MFE estimate serves here as a reference a posteriori error estimate. The second procedure only uses the piecewise affine potential reconstruction $s_{h}$ of Definition 3.8, which allows for the simple matrix form (3.24) of the estimators. The last procedure is definitely the easiest choice in practice, where only the already available element matrices $\widehat{\mathbb{A}}_{K}$ are used and there is no need to construct the mixed finite element matrices $\widehat{\mathbb{A}}_{\mathrm{MFE}, K}$ via (3.12).

In Figure 5 we compare the actual and predicted error distributions. Obviously, the energy error and the rigorous triangular MFE estimate distributions match perfectly. The polygonal MFE estimate and the polygonal HFV estimates give similar results and match also well with the energy error. We depict in Figure 6 the error and estimates as a function of the total number of unknowns and the corresponding effectivity indices for a uniform mesh refinement. This is in this test performed as follows: we refine uniformly the triangular submesh $\mathcal{T}_{h}$, giving actually rise to a Delaunay triangulation on each step, and then we merge triangles into polygons. All the three estimators behave in a similar way, with a slight advantage for the 

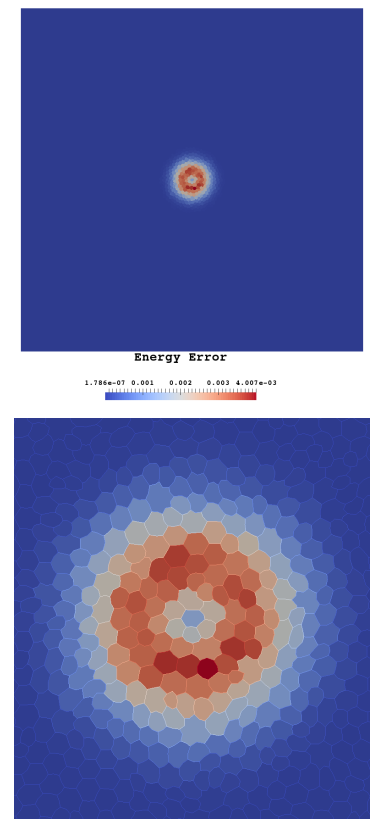

(a) Energy error

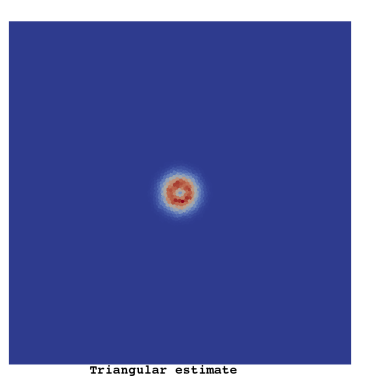

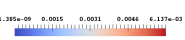

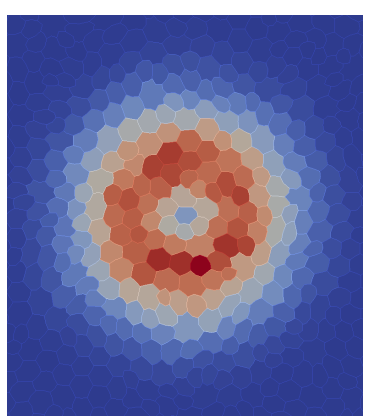

(b) Triangular MFE estimate

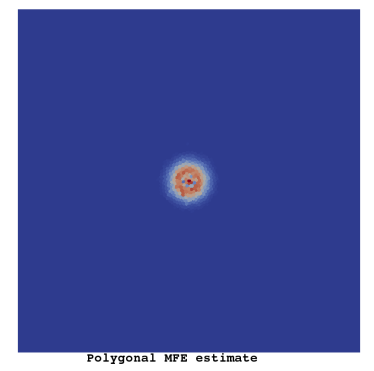

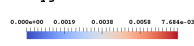

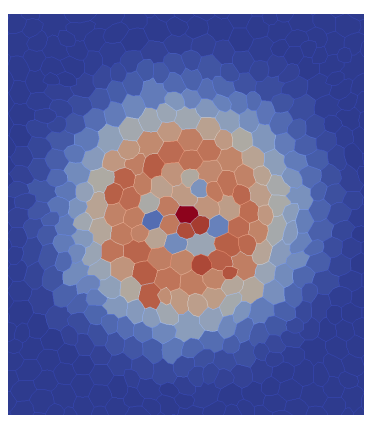

(c) Polygonal MFE estimate

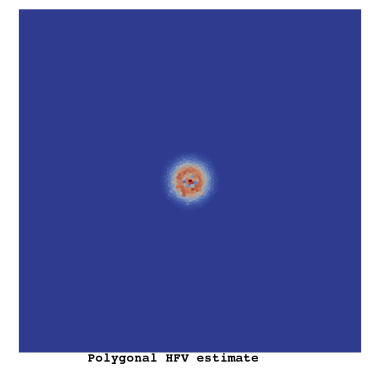

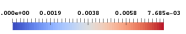

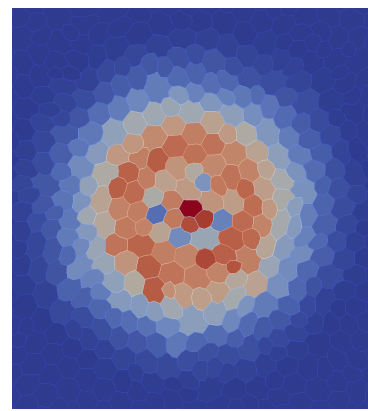

(d) Polygonal HFV estimate

Figure 5: Actual and estimated error distributions, entire domain (top) and center zoom (bottom)
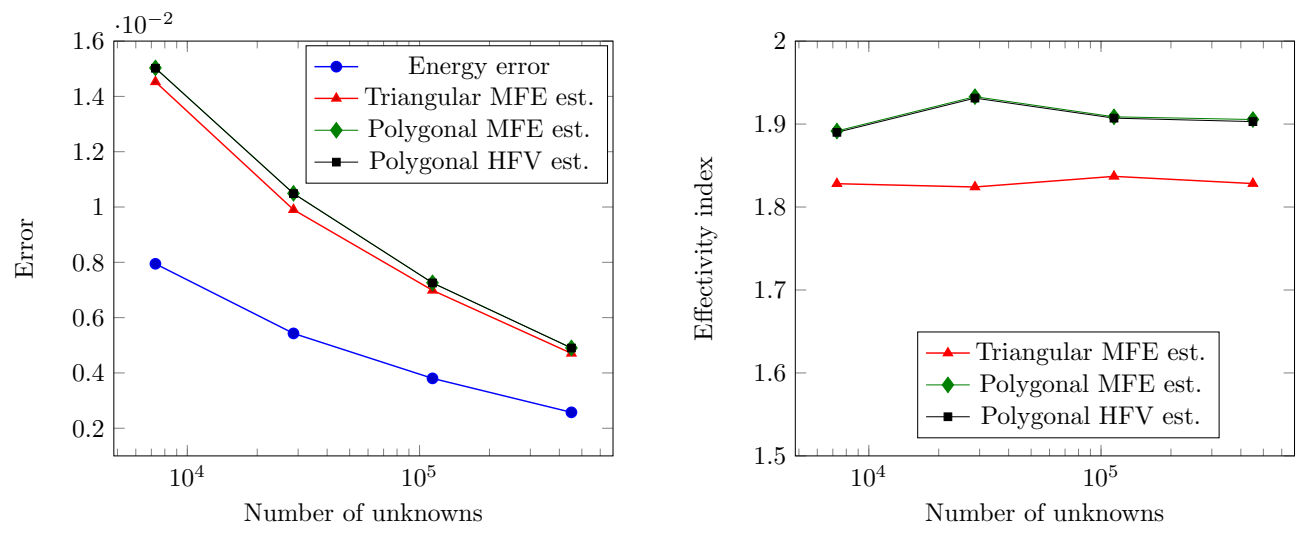

Figure 6: Error and estimators (left) and effectivity indices (right), uniform mesh refinement

triangular MFE estimate. The graphs confirm in particular that replacing the mixed finite element matrix $\widehat{\mathbb{A}}_{\mathrm{MFE}, K}$ in $(3.24)$ by $\widehat{\mathbb{A}}_{K}$ in $(3.28)$ has a very small influence.

Figure 7 shows the results for adaptive mesh refinement, achieved by using the local distribution of the predicted error as an indicator to refine only the cells of the mesh where the error is important. More precisely, we refine the cells $K \in \mathcal{T}_{H}$ such that $\eta_{K} \geq 0.7 \max _{K^{\prime} \in \mathcal{T}_{H}}\left\{\eta_{K^{\prime}}\right\}$. Note that, in this test for the refinement algorithm, we act on the triangular submesh in order to have a Delaunay triangulation on each step and then we merge elements into polygons.

We observe quasi-identical values of the polygonal MFE and polygonal HFV estimates; consequently, we obtain the same number of unknowns at each step of adaptivity and almost identical final energy error for these two estimators. The left part of Figure 7 displays the results where we present the estimators and two energy errors, one resulting with adaptivity based on the polygonal estimates and another with adaptivity based on the triangular MFE estimates. Finally, adaptive mesh refinement leads to both smaller error and better effectivity indices in comparison with uniform mesh refinement. 

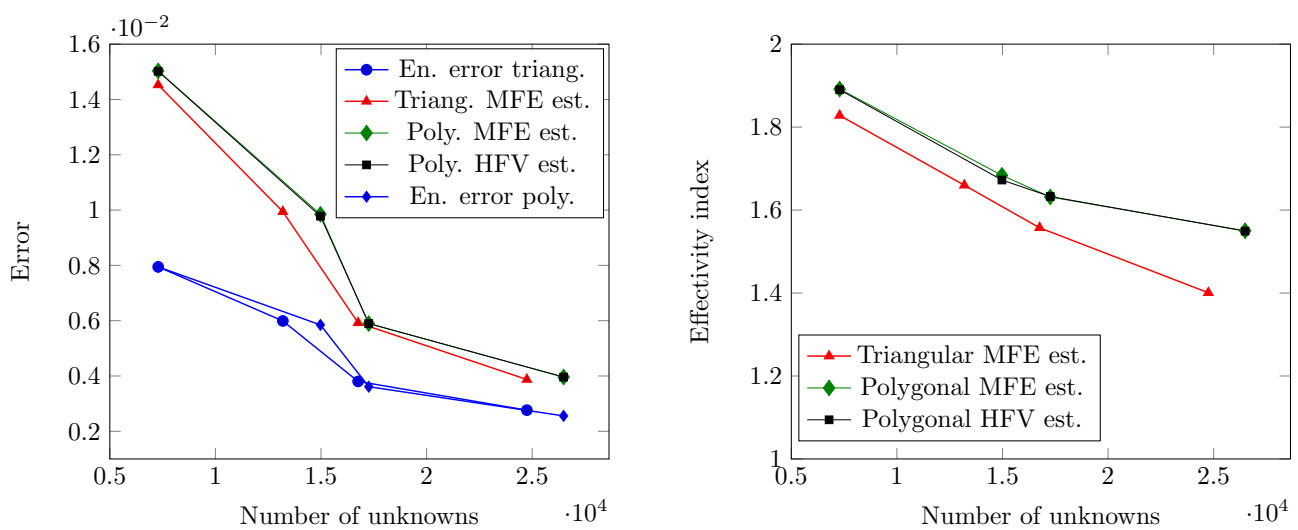

Figure 7: Error and estimators (left) and effectivity indices (right), adaptive mesh refinement

\section{$5 \quad$ A steady nonlinear problem}

As a passage between the model steady linear Darcy flow of Section 3 and the complex multiphase Darcy flow of Section 6, we consider here a steady nonlinear Darcy flow. We showcase that our methodology applies here in a similar simple fashion as in Section 3. We in particular give all the details how inexact linearization and linear solvers can be taken into account, following the concept in Ern and Vohralík [39], giving rise to a simple adaptive inexact Newton method on polytopal meshes. It's particularity is that it gives a guaranteed upper bound on the total error in the fluxes on each linearization and algebraic solver step, distinguishes the different error components, and is obtained by simple multiplications of the same element matrices as in the linear case by vectors of face normal fluxes and potential values immediately available in each lowest-order locally conservative polytopal scheme.

\subsection{Single-phase nonlinear Darcy flow}

For simplicity of exposition, let us consider the following quasi-linear version of (3.1): find $p: \Omega \rightarrow \mathbb{R}$ such that

$$
\begin{array}{rlrl}
-\nabla \cdot \underline{\mathbf{K}}(\nabla p) \nabla p) & =f & & \text { in } \Omega, \\
p=0 & & \text { on } \partial \Omega .
\end{array}
$$

The weak form looks for $p \in H_{0}^{1}(\Omega)$ such that

$$
(\underline{\mathbf{K}}(\nabla p) \nabla p, \nabla v)=(f, v) \quad \forall v \in H_{0}^{1}(\Omega),
$$

and the Darcy velocity is given by

$$
\mathbf{u}:=-\underline{\mathbf{K}}(\nabla p) \nabla p .
$$

We suppose that the nonlinearity can be inverted in the sense that

$$
\mathbf{v}=-\underline{\mathbf{K}}(\mathbf{w}) \mathbf{w} \quad \Longleftrightarrow \mathbf{w}=-\underline{\tilde{\mathbf{K}}}(\mathbf{v}) \mathbf{v}
$$

for all $\mathbf{v}, \mathbf{w} \in \mathbb{R}^{d}$, so that in particular

$$
\nabla p=-\underline{\tilde{\mathbf{K}}}(\mathbf{u}) \mathbf{u} .
$$

We suppose strong monotonicity and Lipschitz-continuity, i.e., that there exist two positive constants $c_{\underline{\mathbf{K}}}, C_{\underline{\mathbf{K}}}$ so that for all $\mathbf{v}, \mathbf{w} \in \mathbb{R}^{d}$,

$$
\begin{aligned}
c_{\tilde{\mathbf{K}}}|\mathbf{v}-\mathbf{w}|^{2} & \leq(\mathbf{v}-\mathbf{w}) \cdot(\underline{\tilde{\mathbf{K}}}(\mathbf{v}) \mathbf{v}-\underline{\tilde{\mathbf{K}}}(\mathbf{w}) \mathbf{w}), \\
|\underline{\tilde{\mathbf{K}}}(\mathbf{v}) \mathbf{v}-\underline{\tilde{\mathbf{K}}}(\mathbf{w}) \mathbf{w}| & \leq C_{\underline{\tilde{\mathbf{K}}}}|\mathbf{v}-\mathbf{w}| .
\end{aligned}
$$


Moreover, we let $\underline{\mathbf{K}}$ and $\underline{\tilde{\mathbf{K}}}$ take symmetric values and, in order to arrive at a simple matrix-vector multiplication form of the estimates, we also suppose that, for all $\mathbf{v}, \mathbf{w} \in \mathbb{R}^{d}$,

$$
c_{\underline{\tilde{\mathbf{K}}}}|\mathbf{v}|^{2} \leq \mathbf{v} \cdot \underline{\tilde{\mathbf{K}}}(\mathbf{w}) \mathbf{v}, \quad|\underline{\tilde{\mathbf{K}}}(\mathbf{w}) \mathbf{v}| \leq C_{\underline{\tilde{\mathbf{K}}}}|\mathbf{v}| .
$$

Note that for a linear problem, $\underline{\tilde{\mathbf{K}}}=\underline{\mathbf{K}}^{-1}, c_{\tilde{\mathbf{K}}}$ is simply the smallest eigenvalue of $\underline{\tilde{\mathbf{K}}}$, i.e., the reciprocal of the largest eigenvalue of $\underline{\mathbf{K}}$, and $C_{\underline{\tilde{\mathbf{K}}}}$ is the largest eigenvalue of $\underline{\tilde{\mathbf{K}}}$, i.e., the reciprocal of the smallest eigenvalue of $\underline{\mathbf{K}}$.

\subsection{Discretization on a polytopal mesh, linearization, and algebraic system solution}

Here again, we can accommodate any discretization of the form of Assumption 3.1, the only difference being that the face normal fluxes $\mathrm{U}$ are now nonlinear functions of the pressures $\mathrm{P}$ that we denote by $\mathrm{U}(\mathrm{P})$. Then, the flux balance (3.4) takes the form, on each polytopal element $K \in \mathcal{T}_{H}$,

$$
\sum_{\sigma \in \mathcal{E}_{K}}(\mathrm{U}(\mathrm{P}))_{\sigma} \mathbf{n}_{K, \sigma} \cdot \mathbf{n}_{\sigma}=(\mathrm{F})_{K} \quad \forall K \in \mathcal{T}_{H},
$$

which gives rise to a system of $\left|\mathcal{T}_{H}\right|$ nonlinear algebraic equations for the $\left|\mathcal{T}_{H}\right|$ unknowns $\mathrm{P}$.

To solve approximately(5.8), one typically employs an iterative linearization such as the Newton or the fixed point method. On a linearization step $k \geq 1$, the flux balance takes the form

$$
\sum_{\sigma \in \mathcal{E}_{K}}\left(\mathrm{U}^{k-1}\left(\mathrm{P}^{k}\right)\right)_{\sigma} \mathbf{n}_{K, \sigma} \cdot \mathbf{n}_{\sigma}=(\mathrm{F})_{K} \quad \forall K \in \mathcal{T}_{H}
$$

where $\mathrm{U}^{k-1}\left(\mathrm{P}^{k}\right)$ are the linearized face normal fluxes, being affine functions of the pressures $\mathrm{P}^{k}$.

Finally, on a step $i \geq 1$ of an iterative algebraic solver applied to this system of linear equations, the flux balance takes the form

$$
\sum_{\sigma \in \mathcal{E}_{K}}\left(\mathrm{U}^{k-1}\left(\mathrm{P}^{k, i}\right)\right)_{\sigma} \mathbf{n}_{K, \sigma} \cdot \mathbf{n}_{\sigma}=(\mathrm{F})_{K}-(\mathrm{R})_{K}^{k, i} \quad \forall K \in \mathcal{T}_{H},
$$

where the constant $(\mathrm{R})_{K}^{k, i}$ is the residual on element $K$, the misfit of $\mathrm{U}^{k-1}\left(\mathrm{P}^{k, i}\right)$ not to satisfy (5.9) defined by $(\mathrm{R})_{K}^{k, i}:=(\mathrm{F})_{K}-\sum_{\sigma \in \mathcal{E}_{K}}\left(\mathrm{U}^{k-1}\left(\mathrm{P}^{k, i}\right)\right)_{\sigma} \mathbf{n}_{K, \sigma} \cdot \mathbf{n}_{\sigma}$. In order to estimate the algebraic error, we will, following [44, 48, 39], also need $j \geq 1$ additional algebraic solver steps in (5.10), giving rise to

$$
\sum_{\sigma \in \mathcal{E}_{K}}\left(\mathrm{U}^{k-1}\left(\mathrm{P}^{k, i+j}\right)\right)_{\sigma} \mathbf{n}_{K, \sigma} \cdot \mathbf{n}_{\sigma}=(\mathrm{F})_{K}-(\mathrm{R})_{K}^{k, i+j} \quad \forall K \in \mathcal{T}_{H} .
$$

A concrete example of a discretization of problem of a form (5.1) by the so-called two-point finite volume scheme with Newton or fixed-point linearization and an arbitrary iterative algebraic solver is given in [39, Section 6.5]; Section 6 below provides another example within a much more complicated context.

\subsection{Fictitious flux and potential reconstructions}

On linearization step $k \geq 1$ and algebraic solver step $i \geq 1$, we dispose from (5.10) of the pressures $\mathrm{P}^{k, i}$. Plugging them into the given nonlinearity and discretization scheme, we obtain the normal face fluxes $\left(\mathrm{U}\left(\mathrm{P}^{k, i}\right)\right)_{\sigma}$ for all $\sigma \in \mathcal{E}_{H}$. Consider now these as the data in definition (3.8) of the spaces $\mathbf{V}_{h, \mathrm{~N}}^{K}$, in place of $(\mathrm{U})_{\sigma}$. As an approximate solution, also termed a fictitious flux reconstruction as it is not necessarily to be constructed in practice, we understand $\mathbf{u}_{h}^{k, i} \in \mathbf{V}_{h, \mathrm{~N}}^{K}$ constructed by (3.9) with $c_{\underline{\mathbf{K}}}^{-1} C_{\underline{\mathbf{K}}}^{2} \underline{\mathbf{I}}$ in place of $\underline{\mathbf{K}}^{-1}$, i.e.

$$
\mathbf{u}_{h}^{k, i}:=c_{\underline{\tilde{\mathbf{K}}}}^{-\frac{1}{2}} C_{\underline{\tilde{\mathbf{K}}}} \arg \min _{\mathbf{v}_{h} \in \mathbf{V}_{h, \mathrm{~N}}^{K}, \nabla \cdot \mathbf{v}_{h}=\text { constant }}\left\|\mathbf{v}_{h}\right\|_{L^{2}(K)} .
$$

This replaces Definition 3.3 in the present nonlinear case. It is important to stress that just like in Section 3, this problem is linear and can be written in a matrix form as in (3.11), just replacing $\underline{\mathbf{K}}^{-1}$ by $c_{\underline{\underline{\mathbf{K}}}}^{-1} C_{\underline{\mathbf{K}}}^{2} \mathbf{I}$. Similarly, as in Section 3.5, the vectors $\mathrm{S}_{K}^{k, i}$ and $\mathrm{S}_{K}^{k, i, \text { ext }}$ of the values of the potential reconstructions in the vertices of the simplicial submesh $\mathcal{T}_{K}$ of the polytopal element $K$ can be immediately obtained. 


\subsection{A simple guaranteed a posteriori estimate distinguishing the error compo- nents}

Let a polytopal cell $K \in \mathcal{T}_{H}$ with its face $\sigma \in \mathcal{E}_{K}$ be fixed. For a given linearization step $k \geq 1$, algebraic step $i \geq 1$, and $j \geq 1$ additional algebraic iterations, define

$$
\begin{aligned}
\left(\mathrm{U}_{K}^{k, i}\right)_{\sigma} & :=\left(\mathrm{U}\left(\mathrm{P}^{k, i}\right)\right)_{\sigma}, \\
\left(\mathrm{U}_{\text {lin, } K}^{k, i}\right)_{\sigma} & :=\left(\mathrm{U}^{k-1}\left(\mathrm{P}^{k, i}\right)\right)_{\sigma}-\left(\mathrm{U}\left(\mathrm{P}^{k, i}\right)\right)_{\sigma}, \\
\left(\mathrm{U}_{\text {alg }, K}^{k, i}\right)_{\sigma} & :=\left(\mathrm{U}^{k-1}\left(\mathrm{P}^{k, i+j}\right)\right)_{\sigma}-\left(\mathrm{U}^{k-1}\left(\mathrm{P}^{k, i}\right)\right)_{\sigma} .
\end{aligned}
$$

The simple choice (5.13c) is motivated by the developments in $[44,48,39]$, where $j$ is typically chosen adaptively. A more involved choice can be made following [57]. Then no additional iterations are needed, the last term in (5.15) below vanishes, and the a posteriori bound is typically slightly more precise.

Let $C_{\mathrm{F}}$ be the constant from the Friedrichs inequality

$$
\|v\|_{L^{2}(\Omega)} \leq C_{\mathrm{F}} h_{\Omega}\|\nabla v\|_{L^{2}(\Omega)}
$$

there holds $1 /(\pi d) \leq C_{\mathrm{F}} \leq 1$, as $C_{\mathrm{F}} h_{\Omega}$ is the square root of the reciprocal of the smallest eigenvalue of the Laplace operator on $\Omega$ with homogenous Dirichlet boundary condition. The main result of this section is:

Theorem 5.1 (Simple a posteriori error estimate distinguishing the error components). Let $\mathbf{u}$ be given by (5.2)-(5.3). For any polytopal discretization of the form (5.8), any linearization (5.9) on step $k \geq 1$, and any algebraic solver (5.10) on step $i \geq 1$, let the flux $\mathbf{u}_{h}^{k, i} \in \mathbf{R T N}_{0}(\Omega)$ be reconstructed following (5.12). Consider $j \geq 1$ additional algebraic solver steps leading to (5.11). Let the element matrices $\widehat{\mathbb{A}}_{\mathrm{MFE}, K}$ and $\widehat{\mathbb{S}}_{\mathrm{FE}, K}$ be defined by (3.12) and (3.18), where one respectively takes the multiples of the identity matrix $c_{\tilde{\mathbf{K}}}^{-1} C_{\tilde{\mathbf{K}}}^{2} \underline{\mathbf{I}}$ in place of $\underline{\mathbf{K}}^{-1}$ and $c_{\tilde{\mathbf{K}}}^{-2} C_{\underline{\mathbf{\mathbf { K }}}} \underline{\mathbf{I}}$ in place of $\underline{\mathbf{K}}$. Let also $\widehat{\mathbb{M}}_{\mathrm{FE}, K}$ be given by (3.20). Let finally the vectors $\mathrm{S}_{K}^{k, i}$ and $\mathrm{S}_{K}^{k, i, e x t}$ be constructed following Section 5.3. Then there holds

$$
c_{\underline{\underline{\mathbf{K}}}}^{\frac{1}{2}}\left\|\mathbf{u}-\mathbf{u}_{h}^{k, i}\right\|_{L^{2}(\Omega)} \leq \eta_{\mathrm{sp}}^{k, i}+\eta_{\mathrm{lin}}^{k, i}+\eta_{\mathrm{alg}}^{k, i}+\eta_{\mathrm{rem}}^{k, i}
$$

with

$$
\eta_{\bullet}^{k, i}=\left\{\sum_{K \in \mathcal{T}_{H}}\left(\eta_{\bullet, K}^{k, i}\right)^{2}\right\}^{\frac{1}{2}}, \quad \bullet=\{\mathrm{sp}, \text { lin, alg, rem }\},
$$

where the (spatial) discretization estimators are given by

$$
\left(\eta_{\mathrm{sp}, K}^{k, i}\right)^{2}:=\left(\mathrm{U}_{K}^{k, i}\right)^{\mathrm{t}} \widehat{\mathbb{A}}_{\mathrm{MFE}, K} \mathrm{U}_{K}^{k, i}+\left(\mathrm{S}_{K}^{k, i}\right)^{\mathrm{t}} \widehat{\mathbb{S}}_{\mathrm{FE}, K} \mathrm{~S}_{K}^{k, i}+2 c_{\tilde{\underline{\mathbf{K}}}}^{-1} C_{\underline{\tilde{\mathbf{K}}}}\left[\left(\mathrm{U}_{K}^{k, i, e x t}\right)^{\mathrm{t}} \mathrm{S}_{K}^{k, i, \text { ext }}-(\mathrm{F})_{K}|K|^{-1} 1^{\mathrm{t}} \widehat{\mathbb{M}}_{\mathrm{FE}, K} \mathrm{~S}_{K}^{k, i}\right],
$$

the linearization estimators by

$$
\left(\eta_{\operatorname{lin}, K}^{k, i}\right)^{2}:=\left(\mathrm{U}_{\operatorname{lin}, K}^{k, i}\right)^{\mathrm{t}} \widehat{\mathbb{A}}_{\mathrm{MFE}, K} \mathrm{U}_{\operatorname{lin}, K}^{k, i},
$$

the algebraic estimators by,

$$
\left(\eta_{\mathrm{alg}, K}^{k, i}\right)^{2}:=\left(\mathrm{U}_{\mathrm{alg}, K}^{k, i}\right)^{\mathrm{t}} \widehat{\mathbb{A}}_{\mathrm{MFE}, K} \mathrm{U}_{\mathrm{alg}, K}^{k, i},
$$

and the algebraic remainder estimators by

$$
\eta_{\mathrm{rem}, K}^{k, i}:=c_{\underline{\tilde{\mathbf{K}}}}^{-\frac{1}{2}} C_{\underline{\tilde{\mathbf{K}}}} C_{\mathrm{F}} h_{\Omega}|K|^{-\frac{1}{2}}\left|(\mathrm{R})_{K}^{k, i+j}\right| .
$$

Proof. The proof follows in spirit of previous works by Prager and Synge [58], Durán and Padra [36], Dari et al. [28], Ainsworth [4], Kim [50], Jiránek et al. [48], and Ern and Vohralík [39, 40]. Let $s \in H_{0}^{1}(\Omega)$ be the solution of

$$
(\underline{\mathbf{K}}(\nabla s) \nabla s, \nabla v)=-\left(\mathbf{u}_{h}^{k, i}, \nabla v\right) \quad \forall v \in H_{0}^{1}(\Omega)
$$


and set $\mathbf{s}:=-\underline{\mathbf{K}}(\nabla s) \nabla s$ similarly to (5.3). By the triangle inequality,

$$
c_{\underline{\underline{\mathbf{K}}}}^{\frac{1}{2}}\left\|\mathbf{u}-\mathbf{u}_{h}^{k, i}\right\|_{L^{2}(\Omega)} \leq c_{\underline{\underline{\mathbf{K}}}}^{\frac{1}{2}}\|\mathbf{u}-\mathbf{s}\|_{L^{2}(\Omega)}+c_{\underline{\underline{\mathbf{K}}}}^{\frac{1}{2}}\left\|\mathbf{s}-\mathbf{u}_{h}^{k, i}\right\|_{L^{2}(\Omega)} .
$$

For the first term above, the strong monotonicity in particular implies

$$
\begin{aligned}
c_{\tilde{\mathbf{K}}}\|\mathbf{u}-\mathbf{s}\|_{L^{2}(\Omega)}^{2} & \leq(\underline{\mathbf{K}}(\nabla p) \nabla p-\underline{\mathbf{K}}(\nabla s) \nabla s, \nabla(p-s)) \\
& =\left(\underline{\mathbf{K}}(\nabla p) \nabla p+\mathbf{u}_{h}^{k, i}, \nabla(p-s)\right) \\
& =(f, p-s)+\left(\mathbf{u}_{h}^{k, i}, \nabla(p-s)\right),
\end{aligned}
$$

Note that by the Lipschitz-continuity (5.6b) together with (5.4),

$$
\|\nabla(p-s)\|_{L^{2}(\Omega)} \leq C_{\underline{\tilde{\mathbf{K}}}}\|\mathbf{u}-\mathbf{s}\|_{L^{2}(\Omega)},
$$

Thus,

$$
\begin{aligned}
c_{\underline{\underline{\mathbf{K}}}}^{\frac{1}{2}}\|\mathbf{u}-\mathbf{s}\|_{L^{2}(\Omega)} & \leq \frac{(f, p-s)+\left(\mathbf{u}_{h}^{k, i}, \nabla(p-s)\right)}{c_{\underline{\underline{\mathbf{K}}}}^{\frac{1}{2}}\|\mathbf{u}-\mathbf{s}\|_{L^{2}(\Omega)}} \\
& \leq c_{\underline{\underline{\mathbf{K}}}}^{-\frac{1}{2}} C_{\underline{\tilde{\mathbf{K}}}} \sup _{v \in H_{0}^{1}(\Omega),\|\nabla v\|_{L^{2}(\Omega)}=1}\left\{(f, v)+\left(\mathbf{u}_{h}^{k, i}, \nabla v\right)\right\} .
\end{aligned}
$$

To estimate (5.19) by a computable quantity, recall first that $\mathbf{u}_{h}^{k, i}$ is given by (5.12) where we define the space $\mathbf{V}_{h, \mathrm{~N}}^{K}$ by the boundary normal fluxes (5.13a); $\mathbf{u}_{h}^{k, i}$ is termed discretization flux reconstruction. Similarly, the linearization error flux reconstruction $\mathbf{u}_{\operatorname{lin}, h}^{k, i}$ is given by (5.12) with the fluxes (5.13b), and the algebraic error flux reconstruction $\mathbf{u}_{\mathrm{alg}, h}^{k, i}$ is given by (5.12) with the fluxes (5.13c). Observe now that

$$
\nabla \cdot\left(\mathbf{u}_{h}^{k, i}+\mathbf{u}_{\mathrm{lin}, h}^{k, i}+\mathbf{u}_{\mathrm{alg}, h}^{k, i}\right)=|K|^{-1} \sum_{\sigma \in \mathcal{E}_{K}}\left(\mathrm{U}^{k-1}\left(\mathrm{P}^{k, i+j}\right)\right)_{\sigma} \mathbf{n}_{K, \sigma} \cdot \mathbf{n}_{\sigma}=\left.f\right|_{K}-|K|^{-1}(\mathrm{R})_{K}^{k, i+j} \quad \forall K \in \mathcal{T}_{H}
$$

by the Green theorem, (5.13), and (5.11). Fix now $v \in H_{0}^{1}(\Omega)$ with $\|\nabla v\|_{L^{2}(\Omega)}=1$. Then adding and subtracting $\left(\mathbf{u}_{\mathrm{lin}, h}^{k, i}+\mathbf{u}_{\mathrm{alg}, h}^{k, i}, \nabla v\right)$ and using the Green theorem together with (5.20),

$$
\begin{aligned}
(f, v)+\left(\mathbf{u}_{h}^{k, i}, \nabla v\right) & =\left(f-\nabla \cdot\left(\mathbf{u}_{h}^{k, i}+\mathbf{u}_{\mathrm{lin}, h}^{k, i}+\mathbf{u}_{\mathrm{alg}, h}^{k, i}\right), v\right)-\left(\mathbf{u}_{\mathrm{lin}, h}^{k, i}+\mathbf{u}_{\mathrm{alg}, h}^{k, i}, \nabla v\right) \\
& =\sum_{K \in \mathcal{T}_{H}}\left\{|K|^{-1}\left((\mathrm{R})_{K}^{k, i+j}, v\right)_{K}-\left(\mathbf{u}_{\operatorname{lin}, h}^{k, i}+\mathbf{u}_{\mathrm{alg}, h}^{k, i}, \nabla v\right)_{K}\right\} .
\end{aligned}
$$

The Cauchy-Schwarz inequality gives

$$
\sum_{K \in \mathcal{T}_{H}}|K|^{-1}\left|\left((\mathrm{R})_{K}^{k, i+j}, v\right)_{K}\right| \leq \sum_{K \in \mathcal{T}_{H}}|K|^{-\frac{1}{2}}\left|(\mathrm{R})_{K}^{k, i+j}\right|\|v\|_{K} \leq\left\{\sum_{K \in \mathcal{T}_{H}}|K|^{-1}\left|(\mathrm{R})_{K}^{k, i+j}\right|^{2}\right\}^{\frac{1}{2}}\|v\|_{\Omega} .
$$

Summing up these developments with the Friedrichs inequality (5.14), infer from (5.19)

$$
c_{\underline{\underline{\mathbf{K}}}}^{\frac{1}{2}}\|\mathbf{u}-\mathbf{s}\|_{L^{2}(\Omega)} \leq c_{\underline{\tilde{\mathbf{K}}}}^{-\frac{1}{2}} C_{\underline{\tilde{\mathbf{K}}}}\left[C_{\mathrm{F}} h_{\Omega}\left\{\sum_{K \in \mathcal{T}_{H}}|K|^{-1}\left|(\mathrm{R})_{K}^{k, i+j}\right|^{2}\right\}^{\frac{1}{2}}+\left\|\mathbf{u}_{\operatorname{lin}, h}^{k, i}\right\|_{L^{2}(\Omega)}+\left\|\mathbf{u}_{\mathrm{alg}, h}^{k, i}\right\|_{L^{2}(\Omega)}\right] .
$$

Finally, to evaluate the middle term above, we use

$$
\left\|\mathbf{u}_{\mathrm{lin}, h}^{k, i}\right\|_{L^{2}(\Omega)}^{2}=\sum_{K \in \mathcal{T}_{H}}\left\|\mathbf{u}_{\mathrm{lin}, h}^{k, i}\right\|_{L^{2}(K)}^{2}
$$


and employ the constant matrix $c_{\underline{\underline{\underline{\underline{K}}}}}^{-1} C_{\underline{\underline{\underline{K}}}}^{2} \underline{\mathbf{I}}$ in place of $\underline{\mathbf{K}}^{-1}$ in Lemma 3.5 on each polytope $K \in \mathcal{T}_{H}$ to infer

$$
c_{\tilde{\tilde{\mathbf{K}}}}^{-1} C_{\underline{\tilde{\mathbf{K}}}}^{2}\left\|\mathbf{u}_{\operatorname{lin}, h}^{k, i}\right\|_{K}^{2}=\left(\mathrm{U}_{\operatorname{lin}, K}^{k, i}\right)^{\mathrm{t}} \widehat{\mathbb{A}}_{\mathrm{MFE}, K} \mathrm{U}_{\operatorname{lin}, K}^{k, i}
$$

for each polytopal element $K \in \mathcal{T}_{H}$, and we proceed similarly for the last term.

We finally estimate the second term in (5.17). For $v \in H_{0}^{1}(\Omega)$ arbitrary, the strong monotonicity in particular leads to

$$
\begin{aligned}
c_{\tilde{\mathbf{K}}}\left\|\mathbf{s}-\mathbf{u}_{h}^{k, i}\right\|_{L^{2}(\Omega)}^{2} & \leq\left(\underline{\mathbf{K}}(\nabla s) \nabla s+\mathbf{u}_{h}^{k, i}, \nabla s+\underline{\tilde{\mathbf{K}}}\left(\mathbf{u}_{h}^{k, i}\right) \mathbf{u}_{h}^{k, i}\right) \\
& =\left(\underline{\mathbf{K}}(\nabla s) \nabla s+\mathbf{u}_{h}^{k, i}, \nabla v+\underline{\tilde{\mathbf{K}}}\left(\mathbf{u}_{h}^{k, i}\right) \mathbf{u}_{h}^{k, i}\right) \\
& \leq\left\|\mathbf{s}-\mathbf{u}_{h}^{k, i}\right\|\left\|\underline{\underline{\mathbf{K}}}\left(\mathbf{u}_{h}^{k, i}\right) \mathbf{u}_{h}^{k, i}+\nabla v\right\|_{L^{2}(\Omega)}
\end{aligned}
$$

by Cauchy-Schwarz,

so that,

$$
\begin{aligned}
c_{\underline{\tilde{\mathbf{K}}}}^{\frac{1}{2}}\left\|\mathbf{s}-\mathbf{u}_{h}^{k, i}\right\|_{L^{2}(\Omega)} & \leq c_{\underline{\tilde{\mathbf{K}}}}^{-\frac{1}{2}} \inf _{v \in H_{0}^{1}(\Omega)}\left\|\underline{\tilde{\mathbf{K}}}\left(\mathbf{u}_{h}^{k, i}\right) \mathbf{u}_{h}^{k, i}+\nabla v\right\|_{L^{2}(\Omega)} \\
& \leq c_{\underline{\tilde{\mathbf{K}}}}^{-\frac{1}{2}}\left\|\underline{\tilde{\mathbf{K}}}\left(\mathbf{u}_{h}^{k, i}\right) \mathbf{u}_{h}^{k, i}+\nabla s_{h}\right\|_{L^{2}(\Omega)}
\end{aligned}
$$

for an arbitrary $s_{h} \in H_{0}^{1}(\Omega)$. Consequently, for each polytopal mesh element $K \in \mathcal{T}_{H}$, employing here also the eigenvalue hypothesis (5.7),

$$
\begin{array}{rlrl}
\left\|\underline{\tilde{\mathbf{K}}}\left(\mathbf{u}_{h}^{k, i}\right) \mathbf{u}_{h}^{k, i}+\nabla s_{h}\right\|_{L^{2}(K)}^{2} & =\left\|\left[\underline{\tilde{\mathbf{K}}}\left(\mathbf{u}_{h}^{k, i}\right)\right]^{\frac{1}{2}}\left(\left[\underline{\tilde{\mathbf{K}}}\left(\mathbf{u}_{h}^{k, i}\right)\right]^{\frac{1}{2}} \mathbf{u}_{h}^{k, i}+\left[\underline{\tilde{\mathbf{K}}}\left(\mathbf{u}_{h}^{k, i}\right)\right]^{-\frac{1}{2}} \nabla s_{h}\right)\right\|_{L^{2}(K)}^{2} \\
& \leq C_{\tilde{\underline{\mathbf{K}}}}\left\|\left[\underline{\tilde{\mathbf{K}}}\left(\mathbf{u}_{h}^{k, i}\right)\right]^{\frac{1}{2}} \mathbf{u}_{h}^{k, i}+\left[\underline{\tilde{\mathbf{K}}}\left(\mathbf{u}_{h}^{k, i}\right)\right]^{-\frac{1}{2}} \nabla s_{h}\right\|_{L^{2}(K)}^{2} \\
& =C_{\underline{\tilde{\mathbf{K}}}}\left\{\left(\underline{\tilde{\mathbf{K}}}\left(\mathbf{u}_{h}^{k, i}\right) \mathbf{u}_{h}^{k, i}, \mathbf{u}_{h}^{k, i}\right)_{K}+2\left(\mathbf{u}_{h}^{k, i}, \nabla s_{h}\right)_{K}+\left(\left[\underline{\tilde{\mathbf{K}}}\left(\mathbf{u}_{h}^{k, i}\right)\right]^{-1} \nabla s_{h}, \nabla s_{h}\right)_{K}\right\} \\
& \leq C_{\underline{\tilde{\mathbf{K}}}}^{2}\left\|\mathbf{u}_{h}^{k, i}\right\|_{K}^{2}+2 C_{\underline{\tilde{\mathbf{K}}}}\left(\mathbf{u}_{h}^{k, i}, \nabla s_{h}\right)_{K}+c_{\underline{\underline{\mathbf{K}}}}^{-1} C_{\tilde{\mathbf{K}}}\left\|\nabla s_{h}\right\|_{K}^{2} . & \text { by }(5.7
\end{array}
$$

For the first term above, we proceed as in (5.22). For the second one, the development (3.26) remains unchanged. Finally, for the third term, we use (3.19) where $\underline{\mathbf{K}}$ has been replaced by $c_{\underline{\underline{\mathbf{K}}}}^{-2} C_{\underline{\mathbf{K}}} \underline{\mathbf{I}}$ to see that

$$
c_{\tilde{\underline{\mathbf{K}}}}^{-2} C_{\underline{\tilde{\mathbf{K}}}}\left\|\nabla s_{h}\right\|_{K}^{2}=\left(\mathrm{S}_{K}^{k, i}\right)^{\mathrm{t}} \widehat{\mathrm{S}}_{\mathrm{FE}, K} \mathrm{~S}_{K}^{k, i} .
$$

The proof is finished by combining the two estimates on $c_{\underline{\tilde{\underline{K}}}}^{\frac{1}{2}}\|\mathbf{u}-\mathbf{s}\|_{L^{2}(\Omega)}$ and $c_{\underline{\tilde{\mathbf{K}}}}^{\frac{1}{2}}\left\|\mathbf{s}-\mathbf{u}_{h}^{k, i}\right\|_{L^{2}(\Omega)}$.

Remark 5.2 (Error structure). From (5.17), (5.19), and (5.23a), we immediately see that

$$
\begin{aligned}
c_{\underline{\hat{\mathbf{K}}}}^{\frac{1}{2}}\left\|\mathbf{u}-\mathbf{u}_{h}^{k, i}\right\|_{L^{2}(\Omega)} & \leq c_{\underline{\tilde{\mathbf{K}}}}^{-\frac{1}{2}} C_{\underline{\tilde{\mathbf{K}}}} \sup _{v \in H_{0}^{1}(\Omega),\|\nabla v\|_{L^{2}(\Omega)}=1}\left(\mathbf{u}-\mathbf{u}_{h}^{k, i}, \nabla v\right)+c_{\underline{\tilde{\underline{K}}}}^{-\frac{1}{2}} \inf _{v \in H_{0}^{1}(\Omega)}\left\|\underline{\tilde{\mathbf{K}}}\left(\mathbf{u}_{h}^{k, i}\right) \mathbf{u}_{h}^{k, i}+\nabla v\right\|_{L^{2}(\Omega)} \\
& \leq 2 c_{\underline{\tilde{\mathbf{K}}}}^{-\frac{1}{2}} C_{\underline{\tilde{\mathbf{K}}}}\left\|\mathbf{u}-\mathbf{u}_{h}^{k, i}\right\|_{L^{2}(\Omega)} ;
\end{aligned}
$$

in the second inequality, we have used properties (5.2)-(5.3) for the first term and (5.5) together with (5.6b) for the second one, accompanied by the Cauchy-Schwarz inequality. This means that the energy error $c_{\underline{\underline{\mathbf{K}}}}^{\frac{1}{2}}\left\|\mathbf{u}-\mathbf{u}_{h}^{k, i}\right\|_{L^{2}(\Omega)}$ is equivalent to the sum of the dual norm of the residual and nonconformity evaluated as the distance to the $H_{0}^{1}(\Omega)$ space. This is an immediate extension from the linear case with an exact algebraic solver giving $\nabla \cdot \mathbf{u}_{h}=f$ to the nonlinear case with inexact linearization and nonlinear solvers, so that $\nabla \cdot \mathbf{u}_{h}^{k, i} \neq f$. It also serves as our motivation for the definition of the error measure $\mathcal{N}^{n, k, i}$ in (6.18a) in the complex multiphase flow below. 
Note that the estimate of Theorem 5.1 still takes the same simple matrix-vector multiplication form of Theorem 3.12, with in particular the same element matrices $\widehat{\mathbb{A}}_{\mathrm{MFE}, K}, \widehat{\mathbb{S}}_{\mathrm{FE}, K}, \widehat{\mathbb{M}}_{\mathrm{FE}, K}$. Similarly to Corollaries 3.13 and 3.14, a further simplification is to replace $\widehat{\mathbb{A}}_{\mathrm{MFE}, K}$ by $\widehat{\mathbb{A}}_{K}$ whenever the latter matrices are available. In practice, the distinction of the different error components is designed to be used in an adaptive inexact Newton algorithm such as Algorithm 6.1 of the next section.

\section{A coupled unsteady nonlinear problem}

In this section, the methodology derived in Sections 3 and 5 is applied to polytopal discretizations of multiphase Darcy flows in porous media. An abstract framework for a posteriori estimates is already developed for two-phase flow in [66, 20], for multiphase flow in [29], and for thermal multiphase flow in [30]. However, these works do not handle general polytopal meshes often encountered in practice and do not address the question of as cheap as possible form of the estimates. In the following, we describe briefly the multiphase model, discuss its discretization by an implicit finite volume scheme, the linearization by the Newton method, and the algebraic solution of the arising linear system. We also explain the required fictitious flux reconstructions and finally develop our simple a posteriori error estimate distinguishing different error components. By decomposing the estimators into the space, time, linearization, and algebraic error components, we can formulate criteria for stopping the iterative algebraic solver and the iterative linearization solver when the corresponding error components do not affect significantly the overall error. Moreover, the spatial and temporal error components can be balanced respectively by time step and space mesh adaptation. In particular, denote by $\eta_{\mathrm{sp}, \mathrm{t}}^{n, k, i}, \eta_{\mathrm{tm}, \mathrm{t}}^{n, k, i}, \eta_{\mathrm{lin}, \mathrm{t}}^{n, k, i}$, and $\eta_{\mathrm{alg}, \mathrm{t}}^{n, k, i}$ respectively the total spatial, temporal, linearization, and algebraic estimators, on time step $n$, linearization step $k$, and algebraic solvers step $i$. Let $\gamma_{\text {lin }}, \gamma_{\text {alg }} \in(0,1)$ and $\Gamma_{\mathrm{tm}}>\gamma_{\mathrm{tm}}>0$ be user-given fixed parameters. Similarly, fix the fractions of cells to refine $\zeta_{\text {ref }}$ and to derefine $\zeta_{\text {deref }}, 0<\zeta_{\text {deref }}<\zeta_{\text {ref }}<1$. Following $[39,66,20,29,30]$ and the references therein, we derive in this section simple estimators to run the following fully adaptive algorithm:

Algorithm 6.1 (adaptive algorithm with time and space mesh refinement and adaptive stopping criteria).

Set $n:=0$.

while $t^{n} \leq t_{\mathrm{F}}$ do $\{$ Time loop $\}$

Set $n:=n+1$.

loop $\{$ Spatial and temporal errors balancing loop

Set $k:=0$.

loop $\{$ Newton linearization loop $\}$

Set $k:=k+1$ and set up the linear system.

Set $i:=0$.

loop $\{$ Algebraic solver loop\}

Perform a step of the iterative algebraic solver and set $i:=i+1$.

Evaluate the different estimators.

Terminate the algebraic solver loop if: $\eta_{\mathrm{alg}, \mathrm{t}}^{n, k, i} \leq \gamma_{\mathrm{alg}} \eta_{\mathrm{sp}, \mathrm{t}}^{n, k, i}$.

end loop

Terminate the Newton linearization loop if: $\eta_{\operatorname{lin}, \mathrm{t}}^{n, k, i} \leq \gamma_{\operatorname{lin}} \eta_{\mathrm{sp}, \mathrm{t}}^{n, k, i}$. end loop

Terminate the spatial and temporal errors balancing loop if

$$
\begin{gathered}
\eta_{\mathrm{sp}, K, \mathrm{t}}^{n, k, i} \geq \zeta_{\mathrm{ref}} \max _{K^{\prime} \in \mathcal{T}_{H}^{n}}\left\{\eta_{\mathrm{sp}, K^{\prime}, \mathrm{t}}^{n, k, i}\right\} \quad \forall K \in \mathcal{T}_{H}^{n} \\
\gamma_{\mathrm{tm}} \eta_{\mathrm{sp}, \mathrm{t}}^{n, k, i} \leq \eta_{\mathrm{tm}, \mathrm{t}}^{n, k, i} \leq \Gamma_{\mathrm{tm}} \eta_{\mathrm{sp}, \mathrm{t}}^{n, k, i}
\end{gathered}
$$

else

Refine the cells $K \in \mathcal{T}_{H}^{n}$ such that $\eta_{\mathrm{sp}, K, \mathrm{t}}^{n, k, i} \geq \zeta_{\text {ref }} \max _{K^{\prime} \in \mathcal{T}_{H}^{n}}\left\{\eta_{\mathrm{sp}, K^{\prime}, \mathrm{t}}^{n, k, i}\right\}$.

Derefine the cells $K \in \mathcal{T}_{H}^{n}$ such that $\eta_{\mathrm{sp}, K, \mathrm{t}}^{n, k, i} \leq \zeta_{\text {deref }} \max _{K^{\prime} \in \mathcal{T}_{H}^{n}}\left\{\eta_{\mathrm{sp}, K^{\prime}, \mathrm{t}}^{n, k, i}\right\}$.

Refine the time step if $\eta_{\mathrm{tm}, \mathrm{t}}^{n, k, i}>\Gamma_{\mathrm{tm}} \eta_{\mathrm{sp}, \mathrm{t}}^{n, k}$, derefine the time step if $\gamma_{\mathrm{tm}} \eta_{\mathrm{sp}, \mathrm{t}}^{n, k, i}>\eta_{\mathrm{tm}, \mathrm{t}}^{n, k, i}$.

end loop

Update data.

end while 


\subsection{Multiphase Darcy flow}

We consider the generalization of Coats' formulation [26] of multiphase Darcy flow models to an arbitrary number of phases by [43]. The same formulation has already been used in [29], so we only give a concise presentation. Let $\mathcal{P}=\{p\}$ be the set of phases, $\mathcal{C}=\{c\}$ the set of components, and, for a given phase $p \in \mathcal{P}$, let $\mathcal{C}_{p} \subset \mathcal{C}$ be the set of its components. $S_{p}$ then denotes the saturation of the phase $p$ and $C_{p, c}$ the molar fraction of the component $c$ in the phase $p$. For a given component $c \in \mathcal{C}$, denote by $\mathcal{P}_{c}$ the set of the phases which contain $c$.

We denote by $P$ the reference pressure such that the phase pressures $P_{p}, p \in \mathcal{P}$, are expressed as

$$
P_{p}:=P+P_{\mathrm{c}_{p}}\left(S_{p}\right),
$$

where $P_{\mathrm{c}_{p}}$ is a given generalized capillary pressure function. We collect the unknowns of the model in the vector $\mathbf{X}:=\left(P,\left(S_{p}\right)_{p \in \mathcal{P}},\left(C_{p, c}\right)_{p \in \mathcal{P}, c \in \mathcal{C}_{p}}\right)$. We denote by $\phi$ the porosity of the medium and by $\underline{\mathbf{K}}$ the permeability tensor. For a phase $p \in \mathcal{P}, k_{\mathrm{r}, p}$ is the relative permeability, $\mu_{p}$ is the dynamic viscosity, $\zeta_{p}$ is the molar density, $\rho_{p}$ is the mass density, and $\nu_{p}$ is the mobility given by $\nu_{p}:=\zeta_{p} \frac{k_{r, p}}{\mu_{p}}$; for simplicity, we suppose here that only $k_{\mathrm{r}, p}$ (and consequently $\nu_{p}$ ) can be functions of the unknown saturations $S_{p}$, the other data being given as constants on each space-time element of the mesh introduced below. More general cases can be treated as in [29].

The system of governing equations is posed in $\Omega \times\left(0, t_{\mathrm{F}}\right)$ by

$$
\partial_{t} l_{c}+\nabla \cdot \boldsymbol{\theta}_{c}=q_{c}, \quad \forall c \in \mathcal{C},
$$

where $q_{c}$, for simplicity piecewise constant on the space-time meshes introduced below, denotes a source or sink and $l_{c}$ is the amount (in moles) of component $c$ per unit volume,

$$
l_{c}=\phi \sum_{p \in \mathcal{P}_{c}} \zeta_{p} S_{p} C_{p, c} .
$$

In (6.2), for each $c \in \mathcal{C}$, the component flux $\boldsymbol{\theta}_{c}$ has the following expression:

$$
\boldsymbol{\theta}_{c}:=\sum_{p \in \mathcal{P}_{c}} \boldsymbol{\theta}_{p, c}, \quad \boldsymbol{\theta}_{p, c}:=\boldsymbol{\theta}_{p, c}(\mathbf{X})=\nu_{p} C_{p, c} \mathbf{v}_{p}\left(P_{p}\right),
$$

where for all $p \in \mathcal{P}, \mathbf{v}_{p}\left(P_{p}\right)$ represents the average phase velocity given by Darcy's law,

$$
\mathbf{v}_{p}\left(P_{p}\right):=-\underline{\mathbf{K}}\left(\nabla P_{p}+\rho_{p} g \nabla z\right) .
$$

Here $g$ is the gravitation acceleration constant. Note at this occasion that only the first part of the Darcy velocity,

$$
\mathbf{u}_{p}\left(P_{p}\right):=-\underline{\mathbf{K}} \nabla P_{p},
$$

is a gradient of the phase pressure. We will use this observation in our error measure below. We assume that no-flow boundary conditions are prescribed for all the component fluxes,

$$
\boldsymbol{\theta}_{c} \cdot \mathbf{n}_{\Omega}=0 \quad \text { on } \partial \Omega \times\left(0, t_{\mathrm{F}}\right) \quad \forall c \in \mathcal{C},
$$

where $\partial \Omega$ denotes the boundary of $\Omega$ and $\mathbf{n}_{\Omega}$ its unit outward normal. At $t=0$ we prescribe the initial amount of each component,

$$
l_{c}(\cdot, 0)=l_{c}^{0} \quad \forall c \in \mathcal{C} .
$$

The previous PDEs is supplemented by a system of algebraic equations imposing the volume conservation

$$
\sum_{p \in \mathcal{P}} S_{p}=1,
$$

the conservation of the quantity of matter

$$
\sum_{c \in \mathcal{C}_{p}} C_{p, c}=1 \quad \forall p \in \mathcal{P},
$$


and local thermodynamic equilibrium expressed by

$$
\sum_{c \in \mathcal{C}}\left(\left|\mathcal{P}_{c}\right|-1\right)=\sum_{p \in \mathcal{P}}\left|\mathcal{C}_{p}\right|-|\mathcal{C}|
$$

equalities of fugacities, see [29] for more details.

\subsection{Finite volume discretization on a polytopal mesh}

To discretize our model, we choose a fully implicit numerical scheme based on phase-upwind and multi-point flux approximation L-method, see [2]. This method, in the steady linear case, fits our Assumption 3.1, but is not of the form of Assumption 3.2. Among many variants of the MPFA methods, the L-method seems to be a good choice for adaptive mesh refinement. Actually, for the L-method, the treatment of hanging nodes is straightforward, see [69]. In order to define this numerical scheme, we start by giving some notations for the space-time mesh. In addition to Section 2 for the space mesh, we let $\left(\tau_{n}\right)_{1 \leq n \leq N}$ denote a sequence of positive real numbers corresponding to the discrete time steps such that $t_{\mathrm{F}}=\sum_{n=1}^{N} \tau_{n}$. We consider the discrete times $\left(t^{n}\right)_{0<n \leq N}$ such that $t^{0}:=0$ and, for $1 \leq n \leq N, t^{n}:=\sum_{i=1}^{n} \tau_{i}$; then we define the time intervals $I_{n}:=\left(t^{n-1}, t^{n}\right)$.

Let $\left(\mathcal{T}_{H}^{n}\right)_{0 \leq n \leq N}$ denote a family of meshes of the space domain $\Omega$ defined in the sense of Section 2 ; we suppose that $\mathcal{T}_{H}^{n}$ has been obtained from $\mathcal{T}_{H}^{n-1}$ by refinement of some elements and by coarsening of some other. For all $0 \leq n \leq N$, we define $\mathcal{E}_{H}^{n}, \mathcal{E}_{h}^{n}, \mathcal{E}_{H, h}^{n}, \mathcal{E}_{K, h}^{n, \text { ext }}$, and $\mathcal{E}_{K, h}^{n, \text { int }}$ similarly to Section 2. The key idea of the MPFA L-method is to express fluxes as a linear combination of cell pressures, using one value per cell. For all $1 \leq n \leq N$, we let $\mathbf{X}_{\mathcal{T}_{H}}^{n}:=\left(\mathbf{X}_{K}^{n}\right)_{K \in \mathcal{T}_{H}^{n}}$, with $\mathbf{X}_{K}^{n}:=\left(P_{K}^{n},\left(S_{p, K}^{n}\right)_{p \in \mathcal{P}},\left(C_{p, c, K}^{n}\right)_{p \in \mathcal{P}, c \in \mathcal{C}_{p}}\right)$ be the vector of discrete unknowns. System 6.2 is then discretized as follows: for all time steps $1 \leq n \leq N$, all polytopal cells $K \in \mathcal{T}_{H}^{n}$, and each component $c \in \mathcal{C}$, we require

$$
\frac{|K|}{\tau^{n}}\left(l_{c, K}\left(\mathbf{X}_{K}^{n}\right)-l_{c, K}\left(\mathbf{X}_{K}^{n-1}\right)\right)+\sum_{\sigma \in \mathcal{E}_{K} \cap \mathcal{E}_{H}^{\text {int }}} \theta_{c, K, \sigma}\left(\mathbf{X}_{\mathcal{T}_{H}}^{n}\right)=|K| q_{c, K}^{n}
$$

This equation expresses the mass balance for the element $K$. Here $q_{c, K}^{n}$ is the value of $q_{c}$ on element $K$ and time interval $I_{n}$; recall also that $|K|$ stands for the Lebesgue measure of the element $K$. First, in a discrete version of $(6.3)$,

$$
l_{c, K}\left(\mathbf{X}_{K}^{n}\right):=\phi_{K} \sum_{p \in \mathcal{P}_{c}} \zeta_{p, K}^{n} S_{p, K}^{n} C_{p, c, K}^{n}
$$

Next, for each component $c \in \mathcal{C}$, following (6.4), its total flux across the face $\sigma$ results from the sum of the corresponding fluxes for each phase $p \in \mathcal{P}_{c}$, i.e., for all $K \in \mathcal{T}_{H}^{n}$ and all $\sigma \in \mathcal{E}_{K} \cap \mathcal{E}_{H}^{\text {int }}$ with $\sigma=\partial K \cap \partial K^{\prime}$,

$$
\theta_{c, K, \sigma}\left(\mathbf{X}_{\mathcal{T}_{H}}^{n}\right):=\sum_{p \in \mathcal{P}_{c}} \nu_{p, K_{p}^{\uparrow}}^{n} C_{p, c, K_{p}^{\uparrow}}^{n} \theta_{p, K, \sigma}\left(\mathbf{X}_{\mathcal{T}_{H}}^{n}\right), \quad K_{p}^{\uparrow}:= \begin{cases}K & \text { if } \theta_{p, K, \sigma}\left(\mathbf{X}_{\mathcal{T}_{H}}^{n}\right) \geq 0, \\ K^{\prime} & \text { otherwise }\end{cases}
$$

with $C_{p, c, K_{p}^{\uparrow}}^{n}$ and $\nu_{p, K_{p}^{\uparrow}}^{n}$ denoting, respectively, the upstream molar fraction and upstream mobility. In (6.10), we have introduced the multi-point finite volume approximation of the normal component of the average phase velocity on face $\sigma$ given by

$$
\theta_{p, K, \sigma}\left(\mathbf{X}_{\mathcal{T}_{H}}^{n}\right):=\theta_{p, K, \sigma}\left(\left\{\mathbf{X}_{K^{\prime}}^{n}\right\}_{K^{\prime} \in \mathcal{S}_{\sigma}^{L}}\right):=\sum_{K^{\prime} \in \mathcal{S}_{\sigma}^{L}} \tau_{K^{\prime}}^{\sigma}\left(P_{p, K^{\prime}}^{n}+\rho_{p, \sigma}^{n} g z_{K^{\prime}}\right),
$$

where, following (6.1),

$$
P_{p, K}^{n}:=P_{K}^{n}+P_{\mathrm{c}_{p}}\left(S_{p, K}^{n}\right)
$$

and, for all $\sigma \in \mathcal{E}_{K} \cap \mathcal{E}_{H}^{\text {int }}, \mathcal{S}_{\sigma}^{L}$ is a flux L-stencil $\mathcal{S}_{\sigma}^{L} \in \mathcal{T}_{H}^{n}$. Moreover, for all $K^{\prime} \in \mathcal{S}_{\sigma}^{L}, \tau_{K^{\prime}}^{\sigma} \in \mathbb{R}$ is the transmissibility coefficient of the face $\sigma$. Details about choosing the proper L-stencil and computing the corresponding transmissibility coefficients can be found in [2]. Finally, boundary fluxes are set to zero to account for the homogeneous natural boundary conditions. 


\subsection{Linearization and algebraic system solution}

At this stage, we need to solve, at each time step, the system of nonlinear algebraic equations resulting from the discretization (6.8). First, for all times $1 \leq n \leq N$, we apply the Newton linearization generating, for $\mathbf{X}_{\mathcal{T}_{H}}^{n, 0}$ fixed, a sequence $\left(\mathbf{X}_{\mathcal{T}_{H}}^{n, k}\right)_{k \geq 1}$ with $\mathbf{X}_{\mathcal{T}_{H}}^{n, k}$ solution to the following system of linear algebraic equations: for all components $c \in \mathcal{C}$ and all mesh elements $K \in \mathcal{T}_{H}^{n}$,

$$
\sum_{K^{\prime} \in \mathcal{T}_{H}^{n}} \frac{\partial R_{c, K}}{\partial \mathbf{X}_{K^{\prime}}^{n}}\left(\mathbf{X}_{\mathcal{T}_{H}}^{n, k-1}\right) \cdot\left(\mathbf{X}_{K^{\prime}}^{n, k}-\mathbf{X}_{K^{\prime}}^{n, k-1}\right)+R_{c, K}\left(\mathbf{X}_{\mathcal{T}_{H}}^{n, k-1}\right)=0
$$

with, for all $c \in \mathcal{C}$ and all $K \in \mathcal{T}_{H}^{n}$,

$$
R_{c, K}\left(\mathbf{X}_{\mathcal{T}_{H}}^{n}\right):=\frac{|K|}{\tau^{n}}\left(l_{c, K}\left(\mathbf{X}_{K}^{n}\right)-l_{c, K}\left(\mathbf{X}_{K}^{n-1}\right)\right)+\sum_{\sigma \in \mathcal{E}_{K} \cap \mathcal{E}_{H}^{\text {int }}} \theta_{c, K, \sigma}\left(\mathbf{X}_{\mathcal{T}_{H}}^{n}\right)-|K| q_{c, K}^{n}
$$

Second, for time $1 \leq n \leq N$, and a given Newton iteration $k \geq 1$, to approximate the solution of the system (6.12), we use an iterative algebraic solver. Developing the derivatives in (6.12), for $\mathbf{X}_{\mathcal{T}_{H}^{n, k}}^{n, 0}$ fixed, this gives a sequence $\left(\mathbf{X}_{\mathcal{T}_{H}}^{n, k, i}\right)_{i \geq 1}$ solving (6.12) up to the residuals, given for all $c \in \mathcal{C}$ and all $K \in \mathcal{T}_{H}^{n}$ by

$$
R_{c, K}^{n, k, i}:=\frac{|K|}{\tau^{n}}\left(l_{c, K}^{n, k, i}-l_{c, K}\left(\mathbf{X}_{K}^{n-1}\right)\right)+\sum_{\sigma \in \mathcal{E}_{K} \cap \mathcal{E}_{H}^{\mathrm{int}}} \theta_{c, K, \sigma}^{n, k, i}-|K| q_{c, K}^{n},
$$

where the linearization of the amount of component $l_{c, K}\left(\mathbf{X}_{K}^{n, k, i}\right)$ of $(6.9)$ is given by

$$
l_{c, K}^{n, k, i}:=l_{c, K}\left(\mathbf{X}_{K}^{n, k-1}\right)+\frac{\partial l_{c, K}}{\partial \mathbf{X}_{K}^{n}}\left(\mathbf{X}_{K}^{n, k-1}\right) \cdot\left(\mathbf{X}_{K}^{n, k, i}-\mathbf{X}_{K}^{n, k-1}\right),
$$

and where the linearization of the component fluxes $\theta_{c, K, \sigma}\left(\mathbf{X}_{\mathcal{T}_{H}, k}^{n, i}\right)$ of $(6.10)$ is given by

$$
\theta_{c, K, \sigma}^{n, k, i}:=\sum_{p \in \mathcal{P}_{c}}\left\{\nu_{p, K_{p}^{\uparrow}}^{n, k-1} C_{p, c, K_{p}^{\uparrow}}^{n, k-1}\left[\theta_{p, K, \sigma}\left(\mathbf{X}_{\mathcal{T}_{H}^{n}}^{n, k-1}\right)+\sum_{K^{\prime} \in \mathcal{T}_{H}^{n}} \frac{\partial \theta_{p, K, \sigma}}{\partial \mathbf{X}_{K^{\prime}}^{n}}\left(\mathbf{X}_{\mathcal{T}_{H}}^{n, k-1}\right) \cdot\left(\mathbf{X}_{K^{\prime}}^{n, k, i}-\mathbf{X}_{K^{\prime}}^{n, k-1}\right)\right]\right\} .
$$

We will also consider $j \geq 1$ additional algebraic solver steps in (6.13), similarly as we have done it in (5.11).

\subsection{Fictitious flux reconstructions}

Following Sections 3.3 and 5.3, we now define an equivalent of $\mathbf{u}_{h}$ of Definition 3.3 and $\mathbf{u}_{h}^{k, i}$ of $(5.12)$. Here again, it is only needed to define the approximate solution but not for the a posteriori error estimators, so that it is not to be constructed in practical calculations. In what follows, we will systematically use the convention (3.7) (without change of the notation) to define the fluxes of the simplicial submesh faces in case they subdivide the original polytopal faces.

Let a time step $1 \leq n \leq N$, a Newton linearization iteration $k \geq 1$, and an algebraic solver iteration $i \geq 1$ be fixed. For all phases $p \in \mathcal{P}$ and all $K \in \mathcal{T}_{H}^{n}$, define $\left.\mathbf{u}_{p, h}^{n, k, i}\right|_{K} \in \mathbf{R T N}_{0}(K)$ as a discrete counterpart of (6.6) by

$$
\left\langle\mathbf{u}_{p, h}^{n, k, i} \cdot \mathbf{n}_{K}, 1\right\rangle_{\sigma}:=\sum_{K^{\prime} \in \mathcal{S}_{\sigma}^{L}} \tau_{K^{\prime}}^{\sigma} P_{p, K^{\prime}}^{n, k, i}, \quad \forall \sigma \in \mathcal{E}_{K, h}^{n, \mathrm{ext}},
$$

with $\mathbf{u}_{p, h}^{n, k, i} \cdot \mathbf{n}_{\Omega}=\underline{\mathbf{K}} \rho_{p} g \nabla z \cdot \mathbf{n}_{\Omega}$ on $\partial \Omega$ coherently with the homogeneous Neumann boundary condition. Similarly, for all components $c \in \mathcal{C}$ and all $K \in \mathcal{T}_{H}^{n}$, we define $\left.\boldsymbol{\theta}_{c, h}^{n, k, i}\right|_{K} \in \mathbf{R T N}_{0}(K)$ as a discrete counterpart of (6.4) by

$$
\left\langle\boldsymbol{\theta}_{c, h}^{n, k, i} \cdot \mathbf{n}_{K}, 1\right\rangle_{\sigma}:=\sum_{p \in \mathcal{P}_{c}} \nu_{p, K}^{n, k, i} C_{p, c, K}^{n, k, i} \theta_{p, K, \sigma}\left(\mathbf{X}_{\mathcal{T}_{H}}^{n, k, i}\right), \quad \forall \sigma \in \mathcal{E}_{K, h}^{n, \text { ext }},
$$


with $\theta_{p, K, \sigma}$ defined by $(6.11)$, while we set $\boldsymbol{\theta}_{c, h}^{n, k, i} \cdot \mathbf{n}_{\Omega}=0$ on $\partial \Omega$. The other degrees of freedom are then defined via a minimization solve as in (3.9). Note that in (6.16), no upwinding is used; these normal fluxes will be compared below to

$$
\theta_{c, K, \sigma}\left(\mathbf{X}_{\mathcal{T}_{H}}^{n, k, i}\right):=\sum_{p \in \mathcal{P}_{c}} \nu_{p, K_{p}^{\uparrow}}^{n, k, i} C_{p, c, K_{p}^{\uparrow}}^{n, k, i} \theta_{p, K, \sigma}\left(\mathbf{X}_{\mathcal{T}_{H}}^{n, k, i}\right), \quad K_{p}^{\uparrow}:= \begin{cases}K & \text { if } \theta_{p, K, \sigma}\left(\mathbf{X}_{\mathcal{T}_{H}}^{n, k, i}\right) \geq 0 \\ K^{\prime} & \text { otherwise }\end{cases}
$$

which stem from (6.10).

In the present evolutive setting, we further need to let $\mathbf{u}_{p, h \tau}^{n, k, i}$ and $\boldsymbol{\theta}_{c, h \tau}^{n, k, i}$ be affine-in-time on the time interval $I_{n}$, given respectively by $\mathbf{u}_{p, h}^{n, k, i}$ and $\boldsymbol{\theta}_{c, h}^{n, k, i}$ of (6.15)-(6.16) at the current time $t^{n}$, and by $\mathbf{u}_{p, h}^{n-1}$ and $\boldsymbol{\theta}_{c, h}^{n-1}$ obtained in the same way but on time previous time $t^{n-1}$ (here we systematically omit the linearization and algebraic solver indices). Finally, we also need to define $l_{c, h \tau}^{n, k, i}$ as affine-in-time on the interval $I_{n}$, given respectively by the values $l_{c, K}\left(\mathbf{X}_{K}^{n-1}\right)$ and $l_{c, K}\left(\mathbf{X}_{K}^{n, k, i}\right)$ at times $t^{n-1}$ and $t^{n}$, for all $K \in \mathcal{T}_{H}^{n}$.

\subsection{Error via dual norm of the residual and potential nonconformity}

Suppose that a weak solution can be defined as in [29, Assumption 3.1]. Let $X^{n}:=L^{2}\left(I_{n} ; H^{1}(\Omega)\right)$ and set

$$
\|\varphi\|_{X^{n}}^{2}:=\int_{I_{n}} \sum_{K \in \mathcal{T}_{H}^{n}}\left\{h_{K}^{-2}\|\varphi\|_{L^{2}(K)}^{2}+\left\|\underline{\mathbf{K}}^{\frac{1}{2}} \nabla \varphi\right\|_{L^{2}(K)}^{2}\right\} \mathrm{d} t
$$

where $h_{K}$ is the diameter of the cell $K \in \mathcal{T}_{H}^{n}$. Then the distance of the current approximate solution to the exact solution can be evaluated as in [29, Section 4.5.1] via

$$
\mathcal{N}^{n, k, i}:=\left\{\sum_{c \in \mathcal{C}}\left(\mathcal{N}_{c}^{n, k, i}\right)^{2}\right\}^{\frac{1}{2}}+\left\{\sum_{p \in \mathcal{P}}\left(\mathcal{N}_{p}^{n, k, i}\right)^{2}\right\}^{\frac{1}{2}}
$$

where

$$
\mathcal{N}_{c}^{n, k, i}:=\sup _{\varphi \in X^{n},\|\varphi\|_{X^{n}=1}} \int_{I_{n}}\left\{\left(\partial_{t} l_{c}-\partial_{t} l_{c, h \tau}^{n, k, i}, \varphi\right)-\left(\boldsymbol{\theta}_{c}-\boldsymbol{\theta}_{c, h \tau}^{n, k, i}, \nabla \varphi\right)\right\} \mathrm{d} t
$$

and

$$
\mathcal{N}_{p}^{n, k, i}:=\inf _{\delta_{p} \in X^{n}}\left\{\sum_{c \in \mathcal{C}_{p}} \int_{I_{n}}\left\{\sum_{K \in \mathcal{T}_{H}^{n}}\left(\nu_{p, K}^{n, k, i} C_{p, c, K}^{n, k, i}\right)^{2}\left\|\mathbf{u}_{p, h \tau}^{n, k, i}+\underline{\mathbf{K}} \nabla \delta_{p}\right\|_{\underline{\mathbf{K}}^{-\frac{1}{2} ; L^{2}(K)}}^{2}\right\} \mathrm{d} t\right\}^{\frac{1}{2}} .
$$

The first term $(6.18 \mathrm{~b})$ is the dual norm of the residual of the weak formulation for each component on the time interval $I_{n}$, and the second term $(6.18 \mathrm{c})$ evaluates the nonconformity in the phase pressures. Indeed, from (6.6), $\mathbf{u}_{p}\left(P_{p}\right)$ should be minus a gradient of a scalar-valued function, which is not necessarily the case for the discrete $\mathbf{u}_{p, h \tau}^{n, k, i}$ given at time $t^{n}$ by (6.15).

Remark 6.2 (Error measure $\mathcal{N}^{n, k, i}$ ). The error measure $\mathcal{N}^{n, k, i}$ is a natural extension of the energy error from the simpler cases. Indeed, in the steady linear case of Section 3, under the assumption of exact linear solver leading to the flux reconstruction $\mathbf{u}_{h}$ lying in $\mathbf{H}(\operatorname{div}, \Omega)$ and satisfying $\nabla \cdot \mathbf{u}_{h}=f$, one has (3.25) which corresponds to vanishing $(6.18 \mathrm{~b})$ and steady single-phase version of $(6.18 \mathrm{c})$. Similarly, in the steady nonlinear case of Section 5, Remark 5.2 leads to the equivalent of the sum of the terms (6.18b) and (6.18c) in the steady case for the only phase/component present.

\subsection{Simple a posteriori estimate distinguishing the error components}

We now extend the a posteriori estimates proposed in [29, Section 4.5.2] to polytopal meshes and derive their simple form only using local matrix-vector multiplications. In extension of (5.13), we again only rely on the following finite volume face fluxes associated with the polytopal mesh elements $K \in \mathcal{T}_{H}^{n}$ and their 
faces $\sigma \in \mathcal{E}_{K, h}^{n, \text { ext }}$ not lying on the boundary of $\Omega$, for all components $c \in \mathcal{C}$, time levels $n \geq 1$, linearization steps $k \geq 1$, algebraic iterations $i \geq 1$, as well as times $t \in I_{n}$ if necessary:

$$
\begin{aligned}
\left(\mathrm{U}_{K, p}^{n, k, i}\right)_{\sigma} & :=\frac{t-t^{n-1}}{\tau^{n}} \sum_{K^{\prime} \in \mathcal{S}_{\sigma}^{L}} \tau_{K^{\prime}}^{\sigma} P_{p, K^{\prime}}^{n, k, i}+\frac{t^{n}-t}{\tau^{n}} \sum_{K^{\prime} \in \mathcal{S}_{\sigma}^{L}} \tau_{K^{\prime}}^{\sigma} P_{p, K^{\prime}}^{n-1}, \\
\left(\Theta_{\mathrm{upw}, K, c}^{n, k, i}\right)_{\sigma} & :=\theta_{c, K, \sigma}\left(\mathbf{X}_{\mathcal{T}_{H}^{n, k, i}}^{n, k}\right)-\sum_{p \in \mathcal{P}_{c}}\left(\nu_{p, K}^{n, k, i} C_{p, c, K}^{n, k, i}\right) \theta_{p, K, \sigma}\left(\mathbf{X}_{\mathcal{T}_{H}^{n, k}}^{n, k,}\right), \\
\left(\Theta_{\mathrm{tm}, K, c}^{n, k, i}\right)_{\sigma} & :=\frac{t^{n}-t}{\tau^{n}} \sum_{p \in \mathcal{P}_{c}}\left[\nu_{p, K}^{n, k, i} C_{p, c, K}^{n, k, i} \theta_{p, K, \sigma}\left(\mathbf{X}_{\mathcal{T}_{H}}^{n, k, i}\right)-\nu_{p, K}^{n-1} C_{p, c, K}^{n-1} \theta_{p, K, \sigma}\left(\mathbf{X}_{\mathcal{T}_{H}}^{n-1}\right)\right], \\
\left(\Theta_{\text {lin, }, K, c}^{n, k, i}\right)_{\sigma} & :=\theta_{c, K, \sigma}^{n, k, i}-\theta_{c, K, \sigma}\left(\mathbf{X}_{\mathcal{T}_{H}, k, i}^{n, k}\right), \\
\left(\Theta_{\mathrm{alg}, K, c}^{n, k, i}\right)_{\sigma} & :=\theta_{c, K, \sigma}^{n, k, i+j}-\theta_{c, K, \sigma}^{n, k, i} ;
\end{aligned}
$$

we set all these normal fluxes to zero for all faces located on the boundary of $\Omega$ in accordance with the Neumann boundary condition (6.7) and define the local vectors as $\Theta_{\bullet, K, c}^{n, k, i}:=\left\{\left(\Theta_{\bullet, K, c}^{n, k, i}\right)_{\sigma}\right\}_{\sigma \in \mathcal{E}_{K}}, \bullet=$ upw, tm, lin, alg, and similarly for $\left(\mathrm{U}_{K, p}^{n, k, i}\right)_{\sigma}$.

We also need to define the phase pressure reconstructions $\mathrm{S}_{K, p}^{n, k, i}$ and $\mathrm{S}_{K, p}^{\text {ext, }, k, i}$, similarly to the steady linear model in Section 3.5. We use directly the cells pressure values as in Definition 3.8. More precisely, the building blocks are the phase pressure values obtained from the finite volume scheme, the current $P_{p, K}^{n, k, i}$ and the previous time step $P_{p, K}^{n-1}$, that we combine for each time $t \in I_{n}$ to

$$
\frac{t-t^{n-1}}{\tau^{n}} P_{p, K}^{n, k, i}+\frac{t^{n}-t}{\tau^{n}} P_{p, K}^{n-1}
$$

Note that all quantities of (6.19) and (6.20) are readily available from the finite volume discretization of Section 6.2, more precisely on each linearization step $k \geq 1$ and each algebraic solver step $i \geq 1$ as described in Section 6.3.

Let $c_{\mathbf{K}}$ be the smallest eigenvalue of the tensor $\underline{\mathbf{K}}$. Proceeding as for the steady linear problem in Section 3.7 and the steady nonlinear problem in Section 5.4 while noting in particular that the chosen MPFA L-scheme verifies Assumption 3.1 in that it provides one phase pressure unknown per element, one normal flux unknown per face and per each component, and a flux balance, we can extend [29, Corollary 4.5] to:

Theorem 6.3 (Simple a posteriori error estimate distinguishing the error components). Consider the multiphase Darcy flow according to Section 6.1, the finite volume scheme of Section 6.2, and its linearization and algebraic system solution according to Section 6.3. Let the approximate solution be understood in the sense of Section 6.4, and the error be measured in the sense of Section 6.5. Let the element matrices $\widehat{\mathbb{A}}_{\mathrm{MFE}, K}$, $\widehat{\mathbb{S}}_{\mathrm{FE}, K}$, and $\widehat{\mathbb{M}}_{\mathrm{FE}, K}$ be respectively defined by (3.12), (3.18), and (3.20). Let $\mathcal{T}_{H}^{n} \subset \mathcal{T}_{H}^{n-1}$, i.e., there is no coarsening. Then

$$
\mathcal{N}^{n, k, i} \leq\left\{\sum_{c \in \mathcal{C}}\left(\eta_{\mathrm{sp}, c}^{n, k, i}+\eta_{\mathrm{tm}, c}^{n, k, i}+\eta_{\mathrm{lin}, c}^{n, k, i}+\eta_{\mathrm{alg}, c}^{n, k, i}+\eta_{\mathrm{rem}, c}^{n, k, i}\right)^{2}\right\}^{\frac{1}{2}}
$$

with

$$
\eta_{\bullet, c}^{n, k, i}:=\left\{\alpha \bullet \int_{I_{n}} \sum_{K \in \mathcal{T}_{H}^{n}}\left(\eta_{\bullet, K, c}^{n, k, i}\right)^{2} \mathrm{~d} t\right\}^{\frac{1}{2}},
$$

where $\bullet=\mathrm{sp}, \mathrm{tm}, \operatorname{lin}$, alg, rem and $\alpha_{\bullet}:=2$, except for $\alpha_{\mathrm{sp}}:=4$. Here, for $c \in \mathcal{C}$, we prescribe the elementwise spatial estimators

$$
\eta_{\mathrm{sp}, K, c}^{n, k, i}:=\eta_{\mathrm{upw}, K, c}^{n, k, i}+\left\{\sum_{p \in \mathcal{P}_{c}}\left(\eta_{\mathrm{NC}, K, c, p}^{n, k, i}\right)^{2}\right\}^{\frac{1}{2}},
$$


with the nonconformity estimators

$$
\begin{aligned}
\left(\eta_{\mathrm{NC}, K, c, p}^{n, k, i}\right)^{2}:= & \left(\nu_{p, K}^{n, k, i} C_{p, c, K}^{n, k, i}\right)^{2}\left[\left(\mathrm{U}_{K, p}^{n, k, i}\right)^{\mathrm{t}} \widehat{\mathbb{A}}_{\mathrm{MFE}, K} \mathrm{U}_{K, p}^{n, k, i}+\left(\mathrm{S}_{K, p}^{n, k, i}\right)^{\mathrm{t}} \widehat{\mathbb{S}}_{\mathrm{FE}, K} \mathrm{~S}_{K, p}^{n, k, i}\right. \\
& \left.+2\left(\mathrm{U}_{K, p}^{n, k, i}\right)^{\mathrm{t}} \mathrm{S}_{K, p}^{\mathrm{ext}, n, k, i}-2 \sum_{\sigma \in \mathcal{E}_{K}}\left(\mathrm{U}_{K, p}^{n, k, i}\right)_{\sigma}|K|^{-1} 1^{\mathrm{t}} \widehat{\mathbb{M}}{ }_{\mathrm{FE}, K} \mathrm{~S}_{K, p}^{n, k, i}\right]
\end{aligned}
$$

and the upwinding estimators

$$
\left(\eta_{\mathrm{upw}, K, c}^{n, k, i}\right)^{2}:=\left(\Theta_{\mathrm{upw}, K, c}^{n, k, i}\right)^{\mathrm{t}} \widehat{\mathbb{A}}_{\mathrm{MFE}, K}\left(\Theta_{\mathrm{upw}, K, c}^{n, k, i}\right)
$$

together with the temporal estimators

$$
\left(\eta_{\mathrm{tm}, K, c}^{n, k, i}\right)^{2}:=\left(\Theta_{\mathrm{tm}, K, c}^{n, k, i}\right)^{\mathrm{t}} \widehat{\mathbb{A}}_{\mathrm{MFE}, K} \Theta_{\mathrm{tm}, K, c}^{n, k, i}
$$

the linearization estimators

$$
\eta_{\operatorname{lin}, K, c}^{n, k, i}:=\left\{\left(\Theta_{\operatorname{lin}, K, c}^{n, k, i}\right)^{\mathrm{t}} \widehat{\mathbb{A}}_{\mathrm{MFE}, K} \Theta_{\operatorname{lin}, K, c}^{n, k, i}\right\}^{\frac{1}{2}}+h_{K}\left(\tau^{n}\right)^{-1}\left\|l_{c, K}\left(\mathbf{X}_{K}^{n, k, i}\right)-l_{c, K}^{n, k, i}\right\|_{L^{2}(K)},
$$

the algebraic estimators

$$
\eta_{\mathrm{alg}, K, c}^{n, k, i}:=\left\{\left(\Theta_{\mathrm{alg}, K, c}^{n, k, i}\right)^{\mathrm{t}} \widehat{\mathbb{A}}_{\mathrm{MFE}, K} \Theta_{\mathrm{alg}, K, c}^{n, k, i}\right\}^{\frac{1}{2}}+h_{K}\left(\tau^{n}\right)^{-1}\left\|l_{c, K}^{n, k, i+j}-l_{c, K}^{n, k, i}\right\|_{L^{2}(K)},
$$

and the algebraic remainder estimators by

$$
\eta_{\mathrm{rem}, K, c}^{n, k, i}:=\min \left\{C_{\mathrm{F}} h_{\Omega} c_{\underline{\mathbf{K}}^{\frac{1}{2}}}^{-\frac{1}{2}}, h_{K}\right\}|K|^{-\frac{1}{2}}\left|R_{c, K}^{n, k, i+j}\right| .
$$

Proof. In order to obtain an a posteriori error estimate distinguishing the different errors components coming from the space, time, linearization, and algebraic resolutions, we proceed as in [29, Section 4.5]. For all components $c \in \mathcal{C}$, all $n \geq 1, k \geq 1, i \geq 1$, and all polytopal cells $K \in \mathcal{T}_{H}^{n}$, only for the purpose of this proof, we first define some supplementary fictitious flux reconstructions by their normal fluxes over $\partial K$; the other degrees of freedom are again defined via a minimization solve as in (3.9):

- The discretization flux reconstruction $\left.\boldsymbol{\theta}_{\mathrm{disc}, c, h}^{n, k, i}\right|_{K} \in \mathbf{R T N}_{0}(K)$ such that

$$
\left\langle\boldsymbol{\theta}_{\mathrm{disc}, c, h}^{n, k, i} \cdot \mathbf{n}_{K}, 1\right\rangle_{\sigma}:=\theta_{c, K, \sigma}\left(\mathbf{X}_{\mathcal{T}_{H}}^{n, k, i}\right), \quad \forall \sigma \in \mathcal{E}_{K, h}^{n, \mathrm{ext}},
$$

with $\theta_{c, K, \sigma}\left(\mathbf{X}_{\mathcal{T}_{H}}^{n, k, i}\right)$ given by $(6.17)$, while $\boldsymbol{\theta}_{\text {disc }, c, h}^{n, k, i} \cdot \mathbf{n}_{\Omega}=0$ on $\partial \Omega$. Note that $\boldsymbol{\theta}_{\text {disc }, c, h}^{n, k, i}$ only differs from $\boldsymbol{\theta}_{c, h}^{n, k, i}$ given by (6.16) by the use of upwinding.

- The linearization error flux reconstruction $\left.\boldsymbol{\theta}_{\text {lin }, c, h}^{n, k, i}\right|_{K} \in \mathbf{R T N}_{0}(K)$ such that

$$
\left\langle\boldsymbol{\theta}_{\mathrm{lin}, c, h}^{n, k, i} \cdot \mathbf{n}_{K}, 1\right\rangle_{\sigma}=\theta_{c, K, \sigma}^{n, k, i}-\theta_{c, K, \sigma}\left(\mathbf{X}_{\mathcal{T}_{H}}^{n, k, i}\right), \quad \forall \sigma \in \mathcal{E}_{K, h}^{n, \text { ext }}
$$

with $\theta_{c, K, \sigma}^{n, k, i}$ given by (6.14), together with $\boldsymbol{\theta}_{\text {lin }, c, h}^{n, k, i} \cdot \mathbf{n}_{\Omega}=0$ on $\partial \Omega$.

- The algebraic error flux reconstruction $\boldsymbol{\theta}_{\mathrm{alg}, c, h}^{n, k,{ }_{K}} \in \mathbf{R T N}_{0}(K)$ such that

$$
\left\langle\boldsymbol{\theta}_{\mathrm{alg}, c, h}^{n, k, i} \cdot \mathbf{n}_{K}, 1\right\rangle_{\sigma}=\theta_{c, K, \sigma}^{n, k, i+j}-\theta_{c, K, \sigma}^{n, k, i}, \quad \forall \sigma \in \mathcal{E}_{K, h}^{n, \mathrm{ext}}
$$

with $\theta_{c, K, \sigma}^{n, k, i}$ given by (6.14) and $\boldsymbol{\theta}_{\mathrm{alg}, c, h}^{n, k, i} \cdot \mathbf{n}_{\Omega}=0$ on $\partial \Omega$; recall that $j \geq 1$ additional algebraic solver iterations need to be performed in (6.13). 
It crucially follows from (6.13) on algebraic step $i+j$, the different flux reconstructions (6.24), and the Green theorem that there holds, for all $c \in C$,

$$
\left(q_{c, K}^{n}-\frac{l_{c, K}^{n, k, i+j}-l_{c, K}\left(\mathbf{X}_{K}^{n-1}\right)}{\tau^{n}}-\nabla \cdot\left(\boldsymbol{\theta}_{\mathrm{disc}, c, h}^{n, k, i}+\boldsymbol{\theta}_{\mathrm{lin}, c, h}^{n, k, i}+\boldsymbol{\theta}_{\mathrm{alg}, c, h}^{n, k, i}\right), 1\right)_{K}=-R_{c, K}^{n, k, i+j} \quad \forall K \in \mathcal{T}_{H}^{n},
$$

similarly as in (5.20). Let $s_{h \tau}^{n, k, i} \in \mathbb{P}_{1}\left(\mathcal{T}_{h}^{n}\right) \cap H_{0}^{1}(\Omega)$ for any $t \in I_{n}$, be prescribed as in Definition 3.8 from the phase pressures (6.20). Then, following [29, Corollary 4.5] and treating the terms $R_{c, K}^{n, k, i+j}$ either as in (5.21) or as $\eta_{\mathrm{R}, K, c}^{n, k, i}$ in [29], (6.21) holds with (6.22) and with the local estimators given, for all components $c \in \mathcal{C}$ and all $K \in \mathcal{T}_{H}^{n}$, by

$$
\begin{aligned}
\eta_{\mathrm{sp}, K, c}^{n, k, i} & :=\eta_{\mathrm{upw}, K, c}^{n, k, i}+\left\{\sum_{p \in \mathcal{P}_{c}}\left(\eta_{\mathrm{NC}, K, c, p}^{n, k, i}\right)^{2}\right\}^{\frac{1}{2}}, \\
\eta_{\mathrm{NC}, K, c, p}^{n, k, i} & :=\nu_{p, K}^{n, k, i} C_{p, c, K}^{n, k, i}\left\|\mathbf{u}_{p, h \tau}^{n, k, i}+\underline{\mathbf{K}} \nabla s_{h \tau}^{n, k, i}\right\|_{\underline{\mathbf{K}}^{-\frac{1}{2} ; L^{2}(K)}}, \\
\eta_{\mathrm{upw}, K, c}^{n, k, i} & :=\left\|\boldsymbol{\theta}_{\mathrm{disc}, c, h}^{n, k, i}-\boldsymbol{\theta}_{c, h}^{n, k, i}\right\|_{\underline{\mathbf{K}}^{-\frac{1}{2}} ; L^{2}(K)}, \\
\eta_{\mathrm{tm}, K, c}^{n, k, i} & :=\left\|\boldsymbol{\theta}_{c, h}^{n, k, i}-\boldsymbol{\theta}_{c, h \tau}^{n, k, i}\right\|_{\underline{\mathbf{K}}^{-\frac{1}{2}} ; L^{2}(K)}, \\
\eta_{\operatorname{lin}, K, c}^{n, k, i} & :=\left\|\boldsymbol{\theta}_{\operatorname{lin}, c, h}^{n, k, i}\right\|_{\underline{\mathbf{K}}^{-\frac{1}{2} ; L^{2}(K)}}+h_{K}\left(\tau^{n}\right)^{-1}\left\|l_{c, K}\left(\mathbf{X}_{K}^{n, k, i}\right)-l_{c, K}^{n, k, i}\right\|_{L^{2}(K)} \\
\eta_{\mathrm{alg}, K, c}^{n, k, i} & :=\left\|\boldsymbol{\theta}_{\mathrm{alg}, c, h}^{n, k, i}\right\|_{\underline{\mathbf{K}}^{-\frac{1}{2}} ; L^{2}(K)}+h_{K}\left(\tau^{n}\right)^{-1}\left\|l_{c, K}^{n, k, i+j}-l_{c, K}^{n, k, i}\right\|_{L^{2}(K)}, \\
\eta_{\mathrm{rem}, K, c}^{n, k, i} & :=\min \left\{C_{\mathrm{F}} h_{\Omega} c_{\left.\underline{\mathbf{K}}^{\frac{1}{2}}, h_{K}\right\}|K|^{-\frac{1}{2}}\left|R_{c, K}^{n, k, i+j}\right|}\right.
\end{aligned}
$$

The bound (6.21) with the estimators given by (6.22) and (6.23) is now obtained by applying Theorem 3.12 to evaluate the nonconformity estimator given by (6.25b) and by applying Lemma 3.5 to evaluate the upwinding, temporal, linearization, and algebraic estimators of $(6.25 \mathrm{c})-(6.25 \mathrm{f})$.

Remark 6.4 (Coarsening). If a mesh element $K \in \mathcal{T}_{H}^{n}$ has been obtained by coarsening of elements of the previous mesh $\mathcal{T}_{H}^{n-1}$ collected in the set $\mathcal{T}_{H, K}^{n-1}$, then the estimators $\eta_{\mathrm{NC}, K, c, p}^{n, k, i}$ of $(6.23 \mathrm{~b})$ and $\eta_{\mathrm{tm}, K, c}^{n, k, i}$ of $(6.23 \mathrm{~d})$ need to be evaluated using the matrices $\widehat{\mathbb{A}}_{\mathrm{MFE}, K}, \widehat{\mathbb{S}}_{\mathrm{FE}, K}$, and $\widehat{\mathbb{M}}_{\mathrm{FE}, K}$ stemming from a common refinement of the element $K$ by the simplicial meshes $\left.\mathcal{T}_{h}^{n-1}\right|_{\mathcal{T}_{H, K}^{n-1}}$ and $\mathcal{T}_{K}$ and not $\mathcal{T}_{K}$ solely. In this sense Theorem 6.3 also holds when $\mathcal{T}_{H}^{n} \not \subset \mathcal{T}_{H}^{n-1}$.

\section{Numerical experiments: multiphase Darcy flow}

In order to validate the results of Theorem 6.3 and of Remark 6.4 and to test the fully adaptive Algorithm 6.1, we study here two different test cases. The first one is taken from [25], relying on the tenth SPE comparative solution project model. It is an incompressible water-oil two-phase flow problem built on a Cartesian regular geometry. This test corresponds to the layer 85 of SPE10. We choose here first the Cartesian regular mesh so as to compare our new approach with approaches already validated on this type of meshes. The second case is a simulation of a black-oil model. For this test, the problem is built on a three-dimensional corner-point geometry (distorted grids), well-known and most often used in reservoir simulation due to the flexibility to permit a good representation of reservoir description, see [31] and references therein. In what follows, the proposed a posteriori error estimate framework has been implemented in a reservoir prototype simulator [59], a thermal multi-purpose simulator written in $\mathrm{C}++$, which is a part of the next generation IFPEn research simulators based on the Arcane framework [46]. The execution platform is a public computer Intel Core i7, 8 cores, $3.7 \mathrm{Ghz}$ with $16 \mathrm{~GB}$ of memory.

In our computational practice presented below, we perform a nonconforming-type mesh refinement and allow one hanging node per cell face for the space mesh adaptation. Coarsening is then obtained 
by retrogressive cell agglomeration. During the coarsening and refinement phases, we use some upscaling and interpolating mechanisms to ensure the mass balance. Geological data (rock properties like porosity and permeability) are stored at the finest mesh level as data on a cloud of space points. These data are used during mesh adaptation by standard upscaling algorithms to compute the values of the rock model properties on the newly created cells following [54].

\subsection{Two-phase flow problem}

We consider a two-dimensional spatial domain discretized by a grid of $60 \times 220$ rectangular cells of size 6.096 $\mathrm{m}$ in the $x$ direction and $3.048 \mathrm{~m}$ in the $y$ direction. We choose the initial time step as $\tau^{0}=4.32 \times 10^{4} \mathrm{~s}$, which equals to 0.5 days, and the process is simulated to $t_{F}=2000$ days. The reservoir is initially saturated with hydrocarbons and we consider the injection of water by a well located at the center of the grid. Four production wells are placed at the four corners of the domain. Therefore, we have a water component $\mathrm{W}$ and an oil component $\mathrm{O}$ collected in the set of components $\mathcal{C}=\{\mathrm{W}, \mathrm{O}\}$ and two phases $\mathcal{P}=\{\mathrm{w}, \mathrm{o}\}$ corresponding to water and oil. The model is actually simplified in that the components can be identified with the phases, so that $\mathcal{P}_{\mathrm{W}}=\{\mathrm{w}\}$ and $\mathcal{P}_{\mathrm{O}}=\{\mathrm{o}\}, C_{\mathrm{w}, \mathrm{W}}=C_{\mathrm{o}, \mathrm{O}}=1, C_{\mathrm{o}, \mathrm{W}}=C_{\mathrm{o}, \mathrm{W}}=0$, and the vector of unknowns $\mathbf{X}$ reduces to $\left(P,\left(S_{p}\right)_{p \in \mathcal{P}}\right)$. Note that then the accumulation term $l_{c}$ becomes linear in the only unknown $S_{p}$, and one particular consequence is that the second term in the definition (6.23e) of $\eta_{\text {lin, }, K, c}^{n, k, i}$ vanishes. The porosity $\phi$ and the permeability field $\underline{\mathbf{K}}$ (scalar coefficient times an identity matrix) are shown in Figure 8. The other parameters of Section 6.1 are given by (see [25] for more details):

- $\mu_{\mathrm{o}}=10^{-3} \mathrm{~Pa} \cdot \mathrm{s}$ and $\mu_{\mathrm{w}}=0.3 \cdot 10^{-3} \mathrm{~Pa} \cdot \mathrm{s}$,

- $\zeta_{\mathrm{o}}=\zeta_{\mathrm{w}}=1 \mathrm{~mole} \cdot \mathrm{m}^{-3}$,

- $S_{\mathrm{wi}}=S_{\mathrm{or}}=0.2$,

$$
k_{\mathrm{r}, \mathrm{w}}\left(S_{\mathrm{w}}\right)=\left(\frac{S_{\mathrm{w}}-S_{\mathrm{wi}}}{1-S_{\mathrm{wi}}-S_{\mathrm{or}}}\right)^{2} \quad \text { and } \quad k_{\mathrm{r}, \mathrm{o}}\left(S_{\mathrm{o}}\right)=\left(\frac{S_{\mathrm{o}}-S_{\mathrm{or}}}{1-S_{\mathrm{wi}}-S_{\mathrm{or}}}\right)^{2},
$$

- $P_{\mathrm{c}_{p}}\left(S_{p}\right)=0$,

- there is no gravitational force, $z=0$, so that the mass densities $\rho_{p}$ need not be specified.
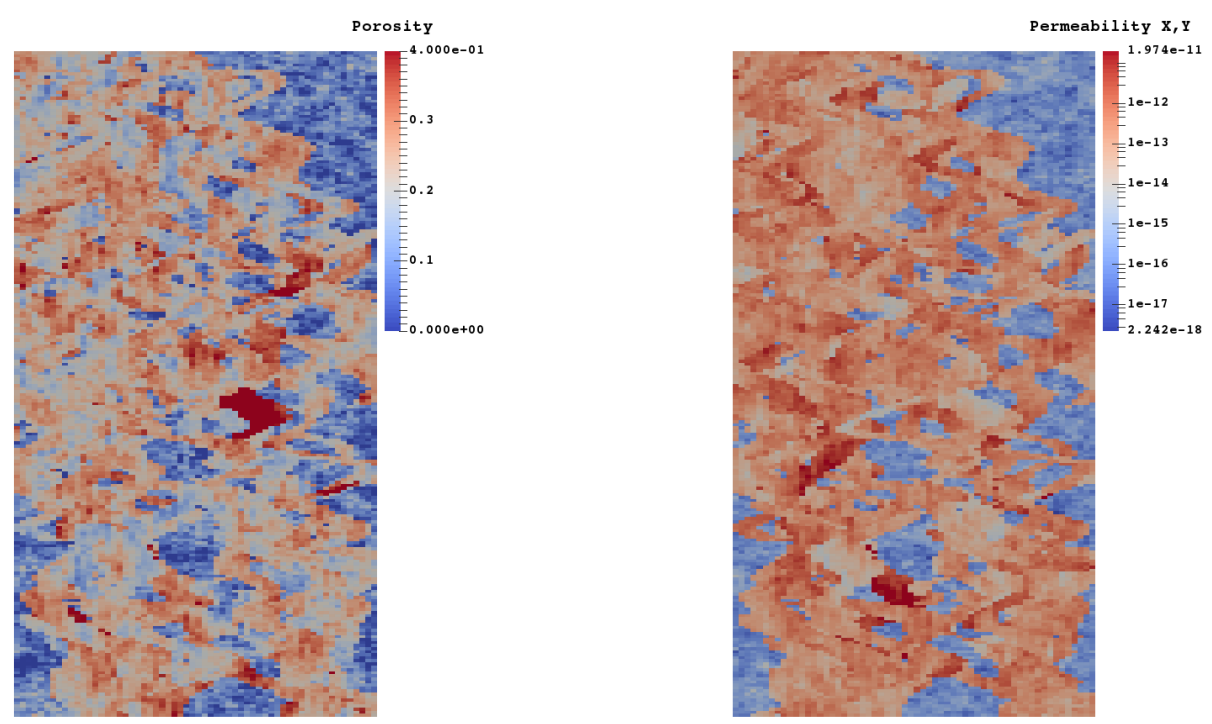

Figure 8: Porosity (left) and permeability (right), 10th SPE case 
We consider a discretization by the implicit multi-point finite volume scheme of Section 6.2 with the Newton linearization detailed in Section 6.3. For the linear solver, we use the Bi-Conjugated Gradient Stabilized (BiCGStab) [62] with an ILU $\{0\}$ preconditioner.

Figure 9 shows, at 500 days of the simulation, the evolution of the approximate water saturation, the spatial estimator computed by the formula on Cartesian grid proposed in [70, Chapter 4], and the spatial estimator given by (6.23a). Similar behavior is observed for the two a posteriori estimators. Furthermore, we see that they both detect well the error following the saturation front despite the strong heterogeneity of the domain.

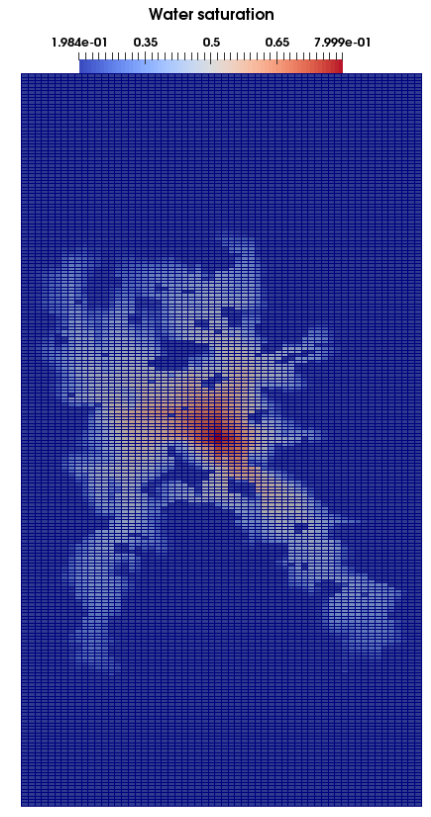

(a) Water saturation

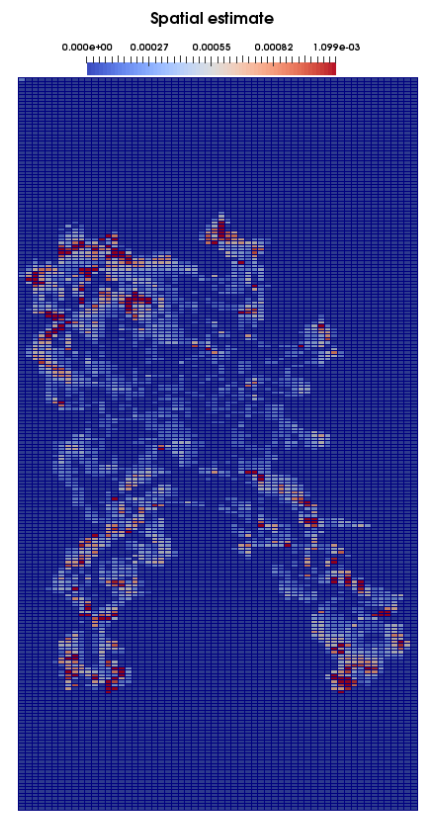

(b) Cartesian estimate of $[45,70]$

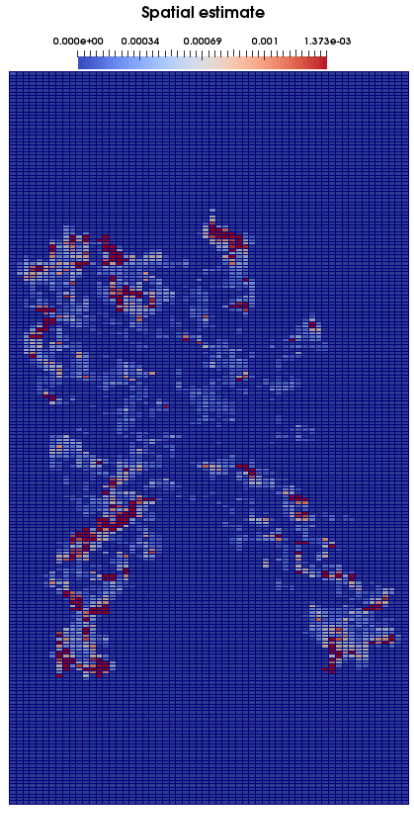

(c) Polygonal simplified estimate (6.23a)

Figure 9: Two-phase flow: results on the fine mesh at 500 days

The previous result motivates an adaptive mesh refinement/coarsening (AMR) strategy based on these estimators. We now verify that the simple a posteriori estimators on polyhedral mesh of Theorem 6.3 give the expected results, while comparing it with the already validated case in [70, Chapter 4]. We focus on the mesh space adaptivity and we do not act on the time step. Actually, in our simulator, the time step size is controlled by the behavior of the Newton algorithm (increased systematically in general, by multiplying by 2 , and divided by 2 if the Newton algorithm diverges), so the space-mesh adaptation only will enable an easy comparison with the non-adaptive code.

We first apply Algorithm 6.1 with $\zeta_{\text {ref }}=0.7, \zeta_{\text {deref }}=0.2$, and "exact" (i.e., negligible-error) algebraic and linearization solvers. On the coarse scale, the domain is discretized by a grid of $30 \times 110$ cells and we allow one refinement level. Figure 10 shows the evolution of the approximate water saturation and of the meshes at two different simulation times. We remark that the refinement follows the saturation front as time evolves. Additionally, the fact that we have a model with highly heterogeneous permeability, we are lead to perform a slow derefinement process in the zone abandoned by the front of water saturation. Figure 11 depicts the cumulated oil rate (left) and the water-cut ${ }^{1}$ (right) during the simulation. We compare there the results on the fine grid, the results on the coarse grid, and the results of the two AMR strategies. We remark that the accuracy of the results on the fine grid is almost recovered by the AMR strategy, and appears much better than the coarse-grid result.

\footnotetext{
${ }^{1}$ The ratio of water produced compared to the volume of total liquids produced.
} 

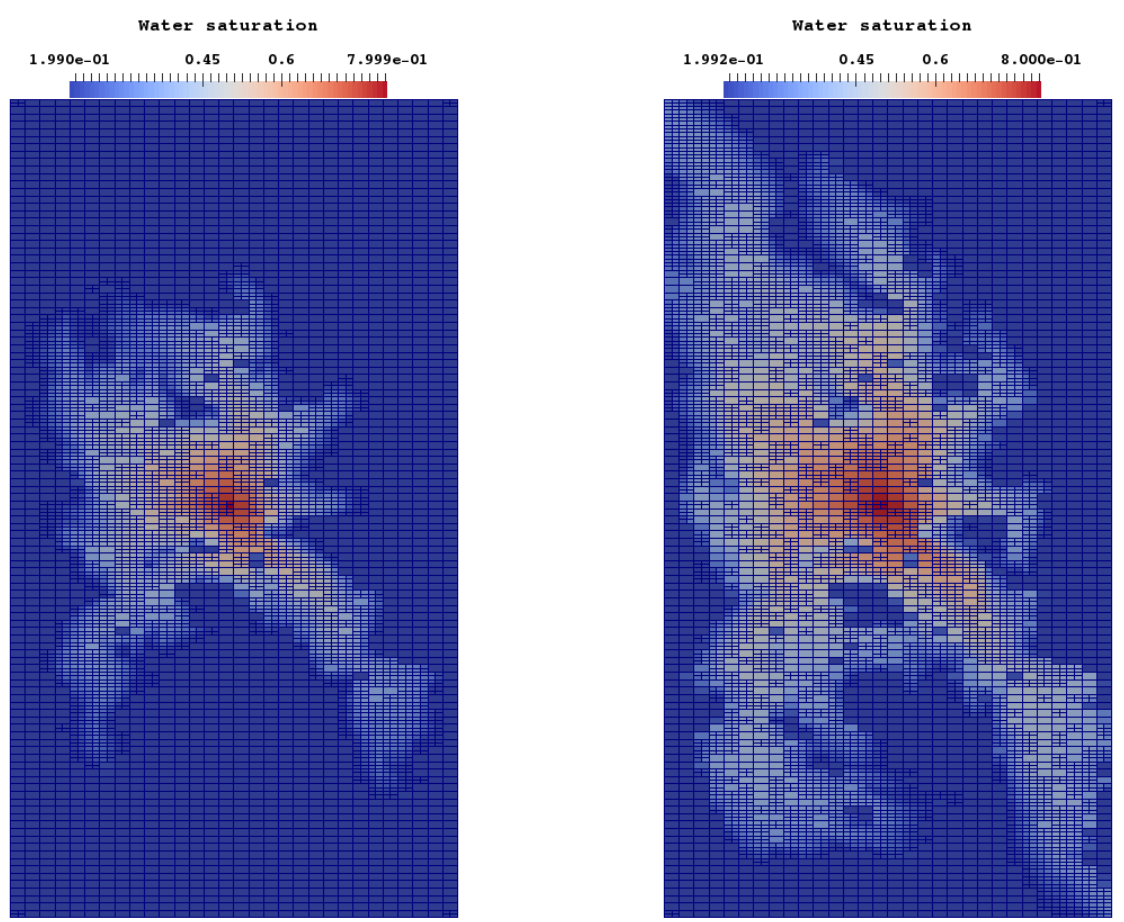

Figure 10: Two-phase flow: results on the adaptive mesh at 400 days and 1100 days
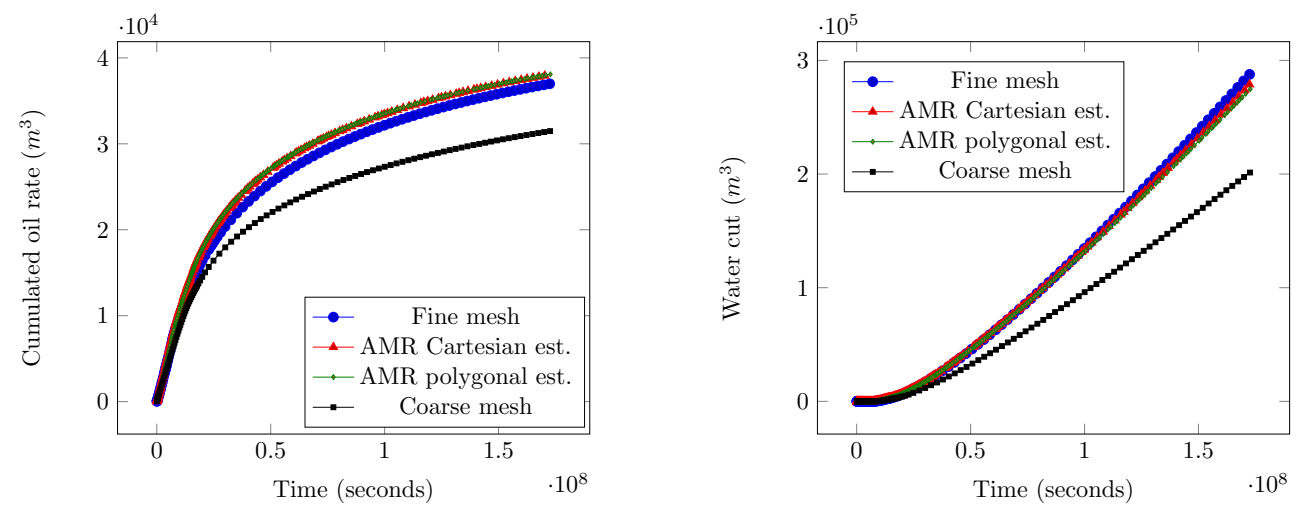

Figure 11: Fine mesh, coarse mesh, and adaptive meshes: cumulated oil rate (left) and water cut (right), two-phase flow

The details of the efficiency of the AMR strategy based on the a posteriori error estimator can finally be appreciated in Table 1. We compare the global CPU time of the simulation for the different strategies. We detail the CPU time spent on the evaluation of the estimators and the mesh adaptation. We remark a low cost of the estimators evaluation compared with the total computation CPU time, thanks to the use of the simple and fast-to-evaluate form of a posteriori error estimates on polygonal meshes of Theorem 6.3. In Table 1, when applying the mesh adaptation, the overall CPU time is the sum of the resolution time, AMR time, and estimators evaluation time. We remark that applying the AMR strategy on this two-dimensional test case leads to a gain factor in the overall CPU time at around 2, for both the polygonal estimate proposed in this paper and the Cartesian estimate already validated on this type of meshes. Note that, however, that the simulation is slightly faster when adapting using the Cartesian estimate, due to the fact that we can here directly compute the RTN basis functions on the rectangular cells. 


\begin{tabular}{|c|c|c|c|c|}
\hline- & Resolution & AMR & Estimators evaluation & Gain factor \\
\hline Fine & $603 \mathrm{~s}$ & - & - & - \\
\hline AMR Cartesian est. & $229 \mathrm{~s}$ & $39 \mathrm{~s}$ & $19 \mathrm{~s}$ & 2.1 \\
\hline AMR polygonal est. & $242 \mathrm{~s}$ & $46 \mathrm{~s}$ & $27 \mathrm{~s}$ & 1.9 \\
\hline
\end{tabular}

Table 1: Fine grid vs. adaptive mesh refinements, two-phase flow

\subsection{Three-phases, three-components problem}

In this section, we present a simulation of a black-oil model. Here, we have three phases constituted by water, oil, and gas, represented by lowercase letters w, o, g as indices, respectively. The oil phase contains two types of components: nonvolatile oil and volatile oil, which we call here oil component and gas component, respectively. This is due to the fact that in this model, the hydrocarbon components are divided into light and heavy components. The light component can dissolve into the liquid oil phase or volatilize in the gas phase according to the pressure and temperature. The gas phase only contains the gas components and the water phase only contains the water component. The components are represented by uppercase letters $\mathrm{W}$ for the water component, $\mathrm{O}$ for the oil component, and $\mathrm{G}$ for the gas component. Therefore, we have a problem with three phases $\mathcal{P}=\{\mathrm{w}, \mathrm{o}, \mathrm{g}\}$ and three components $\mathcal{C}=\{\mathrm{W}, \mathrm{O}, \mathrm{G}\}$.
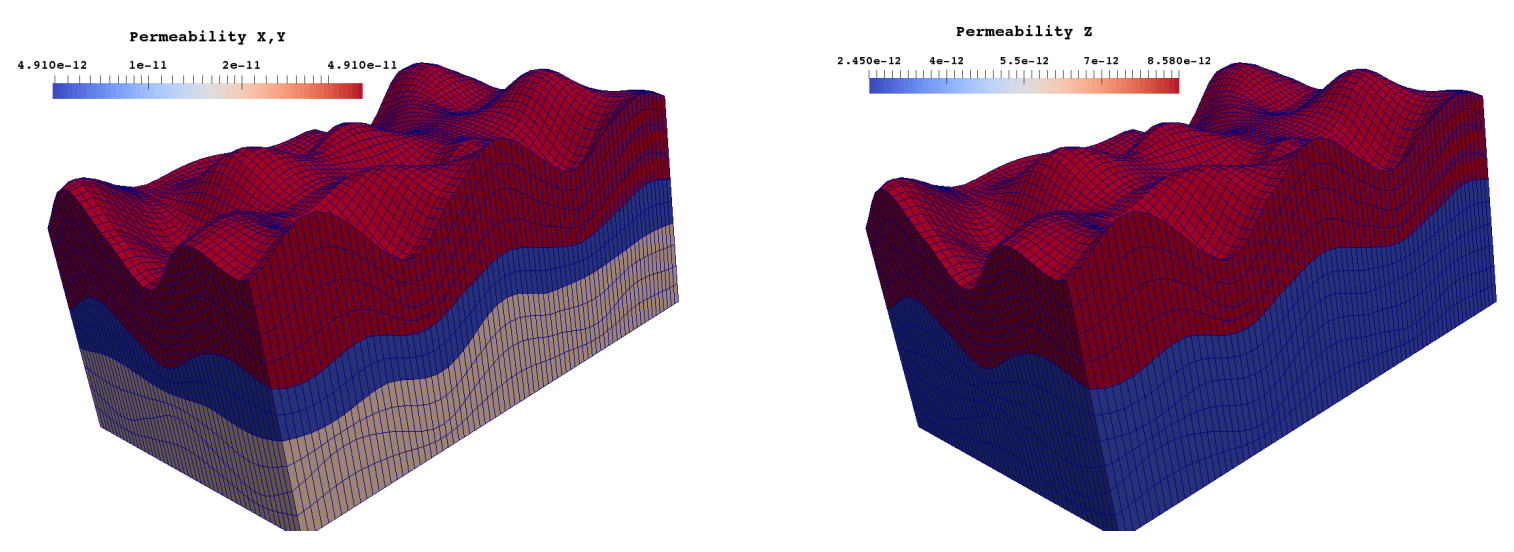

Figure 12: Permeability, black-oil model

\subsubsection{Model setting}

The reservoir considered in this test case is a 3 -dimensional domain $\Omega:=4750 \mathrm{~m} \times 3000 \mathrm{~m} \times 114 \mathrm{~m}$ discretized by a corner-point geometry grid. We consider a heterogeneous anisotropic reservoir with 0.3 porosity and the permeability $\underline{\mathbf{K}}$ in a form of a diagonal matrix with the $x$ and $y$ components identical and forming three horizontal layers, and the $z$ component forming two horizontal layers, see Figure 12 . We consider a gas injection in a reservoir initially unsaturated. A vertical gas injection well perforates a corner of the reservoir in the $z$ direction and a production well is located in the opposite corner. On the fine scale, the domain is discretized by a grid of $76 \times 48 \times 10$ elements and on the coarse scale by a grid of $38 \times 24 \times 5$ cells, leading to one refinement level. The process is simulated to $t_{F}=2000$ days with initial time step $\tau^{0}=4.32 \times 10^{4} \mathrm{~s}$, which equals to 0.5 days. Data, constraints, and pressure-volume properties are adapted from the first SPE comparative solution project model (SPE1) designed to simulate a three-dimensional black-oil reservoir, given in [56, Tables 1,2, and 3]. We consider a discretization by the implicit multi-point finite volume scheme of Section 6.2 with the Newton linearization detailed in Section 6.3. For the linear solver, we again use the BiCGStab [62] with an ILU $\{0\}$ preconditioner. Figure 13 shows the evolution of the gas saturation and the spatial estimator at 1000 days. Note that, for the spatial estimator, the data are normalized by max value in order to have a $[0,1]$ range. We observe that the spatial estimator follows the saturation front though the heterogeneous anisotropic medium with time evolution. 

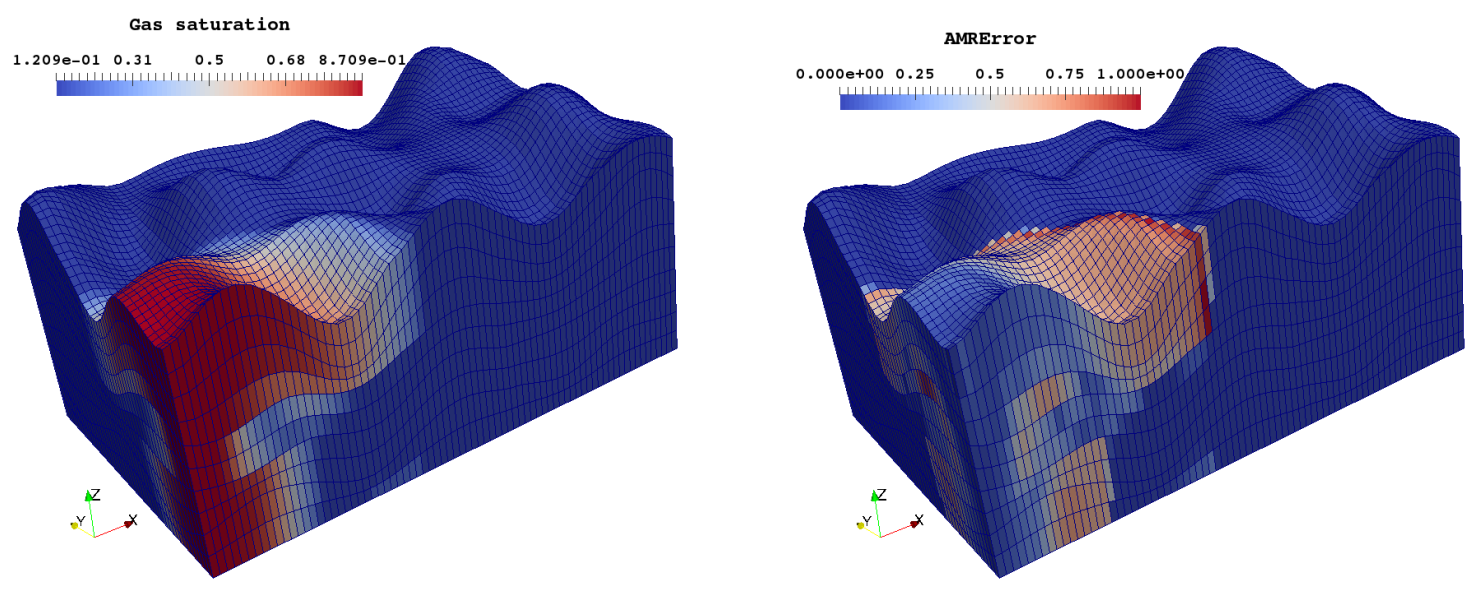

Figure 13: Results on the fine mesh at 1000 days, gas saturation (left) and normalized polygonal estimate (right)

\subsubsection{Adaptive space mesh refinement and stopping criteria for the linear solver}

In the standard resolution of our reservoir prototype simulator, the chosen grid is the fine-scale one and the initial time step, as mentioned before, is chosen as $\tau^{0}=4.32 \times 10^{4} \mathrm{~s}$. As in the test of Section 7.1 , the time step is increased systematically, by multiplying by 2 , but also controlled by the convergence of the Newton linearization loop in such a way that we divide it by 2 if a divergence of the Newton algorithm occurs. We thus stick to this setting and only focus on the stopping criteria for the linear solver (and not for the Newton one) and on mesh adaptivity in space (and not in time). For the adaptive resolution, we start on the coarse-level grid allowing to one refinement level and we fix $\zeta_{\text {ref }}=0.7, \zeta_{\text {deref }}=0.2$ in Algorithm 6.1.

Figure 14 illustrates the evolution of the approximate gas saturation at two different time steps. We remark that the refinement follows the saturation front as time evolves. Additionally, the fact that the light component $\mathrm{G}$ (gas component) can dissolve into the liquid oil phase or volatilize in the gas phase, we are lead to perform some localized refinement in zones abandoned by the front of gas saturation.

We show in the left part of Figure 15, at a fixed time step 500 days and for the first Newton iteration, the evolution of the total estimator $\left(\eta_{\mathrm{sp}, c}^{n, k, i}+\eta_{\mathrm{tm}, c}^{n, k, i}+\eta_{\operatorname{lin}, c}^{n, k, i}++\eta_{\mathrm{alg}, c}^{n, k, i}+\eta_{\mathrm{rem}, c}^{n, k, i}\right)$ with $c=\mathrm{G}$ the gas component and the estimators given in (6.22)-(6.23), the algebraic estimator $\eta_{\text {alg,c }}^{n, k, i}$ of (6.22), (6.23f) (with $\left.j=20\right)$, and the relative algebraic residual given by

$$
\operatorname{err}_{\text {alg }}^{n, k, i}:=\frac{\left\|\mathbb{A}^{n, k-1} \mathbf{X}^{n, k, i}-\mathrm{B}^{n, k-1}\right\|}{\left\|\mathrm{B}^{n, k-1}\right\|}
$$

with $\mathbb{A}^{n, k-1} \mathrm{X}^{n, k}=\mathrm{B}^{n, k-1}$ being the linear system resulting from the $k$-th iteration of the Newton method at time step $t^{n}$, see Section 6.3. For the standard resolution, we stop the algebraic iteration using a fixed threshold $\operatorname{err}_{\mathrm{alg}}^{n, k, i} \leq 10^{-6}$, following our usual practice. In the adaptive resolution based on Algorithm 6.1, we fix $\gamma_{\text {alg }}=10^{-2}$.

We remark, in the left part of Figure 15, that the algebraic estimator steadily decreases, while the total estimator almost stagnates after about a third of total number of iterations necessary to converge using the standard stopping criterion. In the right part of Figure 15, we depict the cumulated number of BiCGStab iterations at each time step (the sum of the necessary number of BiCGStab iterations at each Newton iteration of the time step). We observe a significant gain with the adaptive stopping criterion.

We compare in Figure 16 the number of cells and the cumulated rate of oil production resulting from both the standard and the adaptive resolution. We can observe in the left part of Figure 16 that the adaptive algorithm does not have any significant influence on the accuracy of production. The right part of 

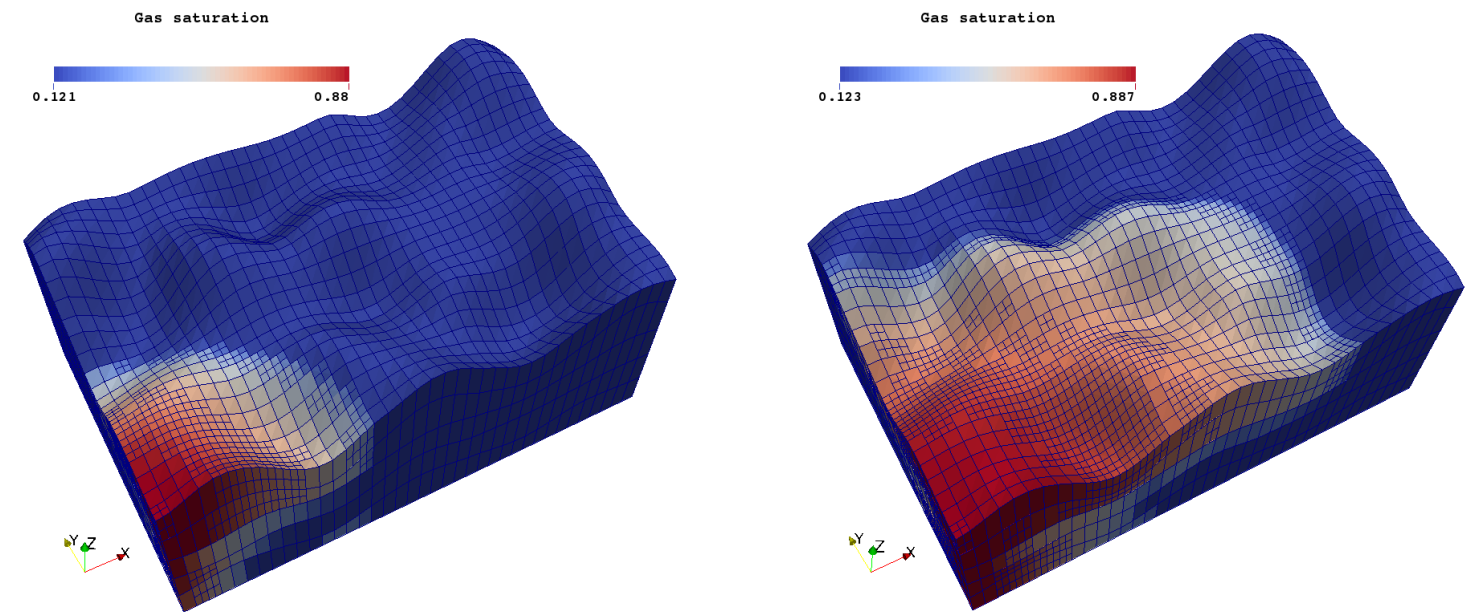

Figure 14: Gas saturation: results on the adaptive mesh at 500 days (left) and 1500 days (right), black-oil model
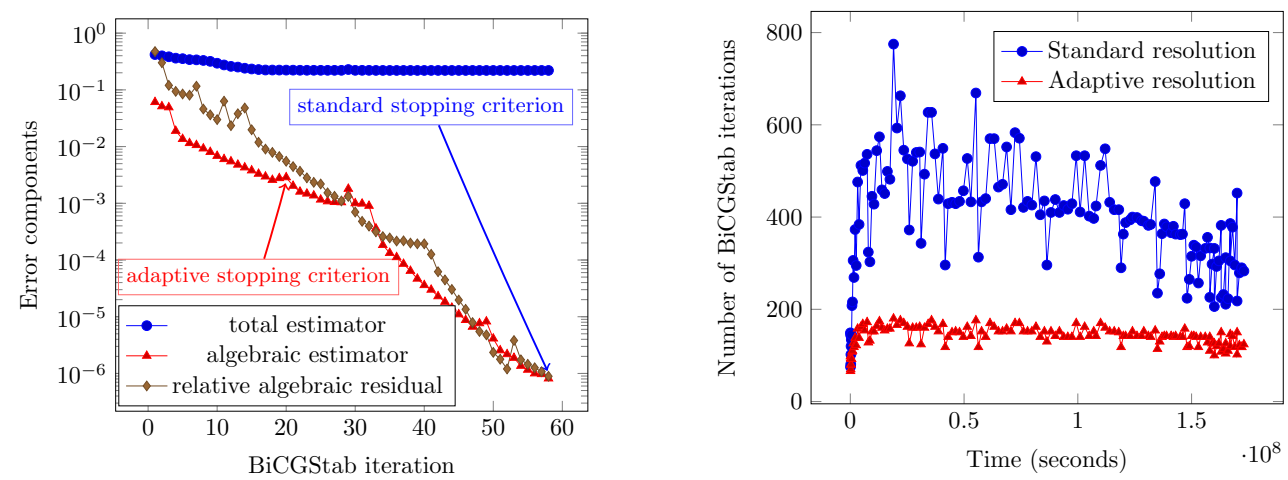

Figure 15: Standard resolution vs. adaptive resolution: total estimator and its algebraic component (left) and number of BiCGStab iterations per time step (right), black-oil model

Figure 16 shows an important reduction in the number of cells via the adaptive resolution compared with the standard one.

The necessary linear solver steps and the different CPU times of the adaptive and standard resolutions are collected in Table 2. It shows that the total number of linear solver steps is reduced by $70 \%$, which is an important gain. An important gain is also observed in the CPU time. We in particular remark that the time spent for the adaptation (AMR and estimators evaluation) only represents a small part of the actual resolution time. Globally, a reduction factor of 3.8 of the overall CPU time is obtained by comparing the adaptive resolution with the standard one. Important increase of this reduction factor is still to be expected when also the Newton solver stopping criteria and time step adaptation are used.

\begin{tabular}{|l|c|c|c|c|c|}
\hline & $\begin{array}{c}\text { Linear solver } \\
\text { steps }\end{array}$ & $\begin{array}{c}\text { Resolution } \\
\text { time }\end{array}$ & $\begin{array}{c}\text { AMR } \\
\text { time }\end{array}$ & $\begin{array}{c}\text { Estimators } \\
\text { evaluation }\end{array}$ & $\begin{array}{c}\text { Gain } \\
\text { factor }\end{array}$ \\
\hline Standard resolution & 66386 & $1023 \mathrm{~s}$ & - & - & - \\
\hline Adaptive resolution & 20184 & $201 \mathrm{~s}$ & $42 \mathrm{~s}$ & $26 \mathrm{~s}$ & 3.8 \\
\hline
\end{tabular}

Table 2: Comparison between standard and adaptive resolutions, black-oil model 

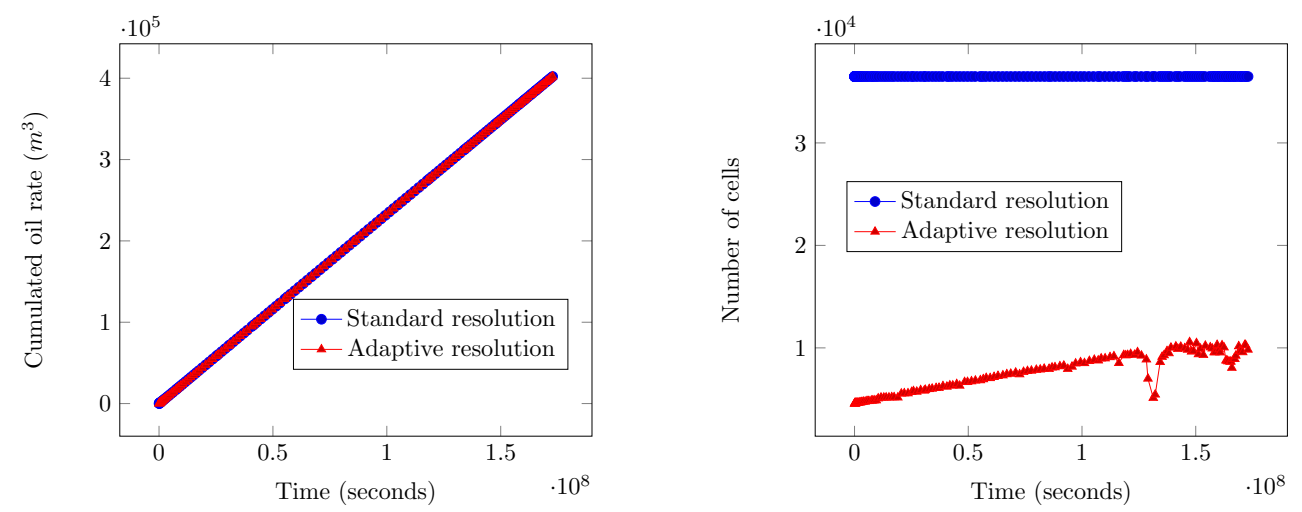

Figure 16: Standard resolution vs. adaptive resolution: cumulated oil rate (left) and number of cells (right), black-oil model

\section{Conclusions}

This paper presents a posteriori error estimates that can be readily implemented into (reservoir engineering) production codes on general polytopal meshes with a minimal overhead. These estimates allow for a very fast evaluation and, according to Theorems 3.12 and 5.1, give a guaranteed control over the error committed in the approximation of the exact Darcy velocity (flux) for the steady linear and steady nonlinear singlephase Darcy flows. Theorem 6.3 then gives an extension of this result to unsteady multiphase Darcy flows. In Theorems 5.1 and 6.3, additionally, all the different error components (time and space discretizations, linearization, algebraic) are identified, leading to a proposition of a fully adaptive Algorithm 6.1 with all adaptive stopping criteria for linear and nonlinear solvers, adaptive time step management, and adaptive mesh refinement. Numerical experiments on real-life problems confirm important speed-ups that can be achieved with our methodology, in addition to the certification of the computed output.

\section{References}

\section{References}

[1] I. Aavatsmark, T. Barkve, Ø. BøE, And T. Mannseth, Discretization on unstructured grids for inhomogeneous, anisotropic media. I. Derivation of the methods, SIAM J. Sci. Comput., 19 (1998), pp. 1700-1716.

[2] I. Aavatsmark, G. T. Eigestad, R. A. Klausen, M. F. Wheeler, and I. Yotov, Convergence of a symmetric MPFA method on quadrilateral grids, Comput. Geosci., 11 (2007), pp. 333-345.

[3] Y. Achdou, C. Bernardi, And F. Coquel, A priori and a posteriori analysis of finite volume discretizations of Darcy's equations, Numer. Math., 96 (2003), pp. 17-42.

[4] M. Ainsworth, Robust a posteriori error estimation for nonconforming finite element approximation, SIAM J. Numer. Anal., 42 (2005), pp. 2320-2341.

[5] P. F. Antonietti, L. Beirão da Veiga, C. Lovadina, And M. Verani, Hierarchical a posteriori error estimators for the mimetic discretization of elliptic problems, SIAM J. Numer. Anal., 51 (2013), pp. 654-675.

[6] Y. Bazilevs, L. Beirão da Veiga, J. A. Cottrell, T. J. R. Hughes, and G. Sangalli, Isogeometric analysis: approximation, stability and error estimates for h-refined meshes, Math. Models Methods Appl. Sci., 16 (2006), pp. 1031-1090. 
[7] L. Beirão DA Veiga, A residual based error estimator for the mimetic finite difference method, Numer. Math., 108 (2008), pp. 387-406.

[8] L. Beirão da Veiga, F. Brezzi, A. Cangiani, G. Manzini, L. D. Marini, and A. Russo, Basic principles of virtual element methods, Math. Models Methods Appl. Sci., 23 (2013), pp. 199-214.

[9] L. Beirão da Veiga, F. Brezzi, L. D. Marini, And A. Russo, Mixed virtual element methods for general second order elliptic problems on polygonal meshes, ESAIM Math. Model. Numer. Anal., 50 (2016), pp. 727-747.

[10] L. BeirÃo DA VeIGA AND G. MANZINI, An a posteriori error estimator for the mimetic finite difference approximation of elliptic problems, Internat. J. Numer. Methods Engrg., 76 (2008), pp. 1696-1723.

[11] - Residual a posteriori error estimation for the virtual element method for elliptic problems, ESAIM Math. Model. Numer. Anal., 49 (2015), pp. 577-599.

[12] P. B. Bochev And J. M. Hyman, Principles of mimetic discretizations of differential operators, in Compatible spatial discretizations, vol. 142 of IMA Vol. Math. Appl., Springer, New York, 2006, pp. $89-119$.

[13] J. Bonelle AND A. ERn, Analysis of compatible discrete operator schemes for elliptic problems on polyhedral meshes, ESAIM Math. Model. Numer. Anal., 48 (2014), pp. 553-581.

[14] J. BREIL AND P.-H. MAIRE, A cell-centered diffusion scheme on two-dimensional unstructured meshes, J. Comput. Phys., 224 (2007), pp. 785-823.

[15] F. Brezzi And M. Fortin, Mixed and hybrid finite element methods, vol. 15 of Springer Series in Computational Mathematics, Springer-Verlag, New York, 1991.

[16] F. Brezzi, K. Lipnikov, and M. Shashkov, Convergence of the mimetic finite difference method for diffusion problems on polyhedral meshes, SIAM J. Numer. Anal., 43 (2005), pp. 1872-1896.

[17] F. Brezzi, K. Lipnikov, and V. Simoncini, A family of mimetic finite difference methods on polygonal and polyhedral meshes, Math. Models Methods Appl. Sci., 15 (2005), pp. 1533-1551.

[18] A. Buffa And C. Giannelli, Adaptive isogeometric methods with hierarchical splines: error estimator and convergence, Math. Models Methods Appl. Sci., 26 (2016), pp. 1-25.

[19] E. Burman AND A. ERn, Continuous interior penalty hp-finite element methods for advection and advection-diffusion equations, Math. Comp., 76 (2007), pp. 1119-1140.

[20] C. CAncÈs, I. S. Pop, And M. VohralÍK, An a posteriori error estimate for vertex-centered finite volume discretizations of immiscible incompressible two-phase flow, Math. Comp., 83 (2014), pp. 153188.

[21] A. Cangiani, E. H. Georgoulis, and P. Houston, hp-version discontinuous Galerkin methods on polygonal and polyhedral meshes, Math. Models Methods Appl. Sci., 24 (2014), pp. 2009-2041.

[22] A. Cangiani, E. H. Georgoulis, T. Pryer, And O. J. Sutton, A posteriori error estimates for the virtual element method, Numer. Math., 137, Issue 4 (2017), pp. 857-893. DOI 10.1007/s00211-0170891-9.

[23] S. H. Christiansen, A construction of spaces of compatible differential forms on cellular complexes, Math. Models Methods Appl. Sci., 18 (2008), pp. 739-757.

[24] — Éléments finis mixtes minimaux sur les polyèdres, C. R. Math. Acad. Sci. Paris, 348 (2010), pp. $217-221$.

[25] M. Christie And M. Blunt, Tenth SPE comparative solution project: A comparison of upscaling techniques, in SPE Reservoir Simulation Symposium, Society of Petroleum Engineers, 2001. 
[26] K. H. CoAts ET AL., Implicit compositional simulation of single-porosity and dual-porosity reservoirs, in SPE Symposium on Reservoir Simulation, Society of Petroleum Engineers, 1989.

[27] B. Cockburn, D. A. Di Pietro, And A. ERn, Bridging the hybrid high-order and hybridizable discontinuous Galerkin methods, ESAIM Math. Model. Numer. Anal., 50 (2016), pp. 635-650.

[28] E. DARI, R. Durán, C. PADra, And V. VAmpa, A posteriori error estimators for nonconforming finite element methods, RAIRO Modél. Math. Anal. Numér., 30 (1996), pp. 385-400.

[29] D. A. Di Pietro, E. Flauraud, M. Vohralík, and S. Yousef, A posteriori error estimates, stopping criteria, and adaptivity for multiphase compositional Darcy flows in porous media, J. Comput. Phys., 276 (2014), pp. 163-187.

[30] D. A. Di Pietro, M. Vohralík, And S. Yousef, An a posteriori-based, fully adaptive algorithm with adaptive stopping criteria and mesh refinement for thermal multiphase compositional flows in porous media, Comput. Math. Appl., 68 (2014), pp. 2331-2347.

[31] Y. Ding AND P. LEMONNIER, Use of corner point geometry in reservoir simulation, Society of Petroleum Engineers, (1995).

[32] J. Droniou, Finite volume schemes for diffusion equations: introduction to and review of modern methods, Math. Models Methods Appl. Sci., 24 (2014), pp. 1575-1619.

[33] J. Droniou And R. Eymard, A mixed finite volume scheme for anisotropic diffusion problems on any grid, Numer. Math., 105 (2006), pp. 35-71.

[34] J. Droniou, R. Eymard, T. Gallouët, And R. Herbin, A unified approach to mimetic finite difference, hybrid finite volume and mixed finite volume methods, Math. Models Methods Appl. Sci., 20 (2010), pp. 265-295.

[35] J. Droniou, R. Eymard, and R. Herbin, Gradient schemes: generic tools for the numerical analysis of diffusion equations, ESAIM Math. Model. Numer. Anal., 50 (2016), pp. 749-781.

[36] R. DURÁN AND C. PADRA, An error estimator for nonconforming approximations of a nonlinear problem, in Finite element methods (Jyväskylä, 1993), vol. 164 of Lecture Notes in Pure and Appl. Math., Dekker, New York, 1994, pp. 201-205.

[37] M. G. EDWARDS, Unstructured, control-volume distributed, full-tensor finite-volume schemes with flow based grids, Comput. Geosci., 6 (2002), pp. 433-452. Locally conservative numerical methods for flow in porous media.

[38] A. ERn AND M. VohralíK, Flux reconstruction and a posteriori error estimation for discontinuous Galerkin methods on general nonmatching grids, C. R. Math. Acad. Sci. Paris, 347 (2009), pp. 441-444.

[39] — Adaptive inexact Newton methods with a posteriori stopping criteria for nonlinear diffusion PDEs, SIAM J. Sci. Comput., 35 (2013), pp. A1761-A1791.

[40] — Polynomial-degree-robust a posteriori estimates in a unified setting for conforming, nonconforming, discontinuous Galerkin, and mixed discretizations, SIAM J. Numer. Anal., 53 (2015), pp. 10581081.

[41] R. Eymard, T. Gallouët, And R. Herbin, Finite volume approximation of elliptic problems and convergence of an approximate gradient, Appl. Numer. Math., 37 (2001), pp. 31-53.

[42] — Discretization of heterogeneous and anisotropic diffusion problems on general nonconforming meshes SUSHI: a scheme using stabilization and hybrid interfaces, IMA J. Numer. Anal., 30 (2010), pp. 1009-1043.

[43] R. Eymard, C. Guichard, R. Herbin, and R. Masson, Vertex-centred discretization of multiphase compositional Darcy flows on general meshes, Computational Geosciences, 16 (2012), pp. 987-1005. 
[44] G. H. Golub And Z. Strakoš, Estimates in quadratic formulas, Numer. Algorithms, 8 (1994), pp. 241-268.

[45] J. M. Gratien, O. Ricois, and S. Yousef, Reservoir simulator runtime enhancement based on a posteriori error estimation techniques, Oil Gas Sci. Technol. Rev. IFP Energies nouvelles, 71 (2016), p. 59 .

[46] G. Grospellier and B. Lelandais, The Arcane development framework, in Proceedings of the 8th workshop on Parallel/High-Performance Object-Oriented Scientific Computing, POOSC '09, New York, NY, USA, 2009, ACM, pp. 4:1-4:11.

[47] R. Herbin And F. Hubert, Benchmark on discretization schemes for anisotropic diffusion problems on general grids, in Finite volumes for complex applications V, ISTE, London, 2008, pp. 659-692.

[48] P. Jiránek, Z. Strakoš, And M. Vohralík, A posteriori error estimates including algebraic error and stopping criteria for iterative solvers, SIAM J. Sci. Comput., 32 (2010), pp. 1567-1590.

[49] O. A. Karakashian And F. Pascal, A posteriori error estimates for a discontinuous Galerkin approximation of second-order elliptic problems, SIAM J. Numer. Anal., 41 (2003), pp. 2374-2399.

[50] K.-Y. Kim, A posteriori error estimators for locally conservative methods of nonlinear elliptic problems, Appl. Numer. Math., 57 (2007), pp. 1065-1080.

[51] R. A. Klausen and T. F. Russell, Relationships among some locally conservative discretization methods which handle discontinuous coefficients, Comput. Geosci., 8 (2004), pp. 341-377 (2005).

[52] Y. Kuznetsov And S. Repin, New mixed finite element method on polygonal and polyhedral meshes, Russian J. Numer. Anal. Math. Modelling, 18 (2003), pp. 261-278.

[53] Y. A. Kuznetsov, Mixed finite element methods on polyhedral meshes for diffusion equations, in Partial differential equations, vol. 16 of Comput. Methods Appl. Sci., Springer, Dordrecht, 2008, pp. 2741.

[54] Y. MesRi AND O. Ricois, Construction process for an improved meshing for the simulation of a reservoir in an underground formation. Patent CA 28886110, 23 03, 2015.

[55] W. F. Mitchell, A collection of $2 D$ elliptic problems for testing adaptive grid refinement algorithms, Applied Mathematics and Computation, 220 (2013), pp. 350-364.

[56] A. S. ODEH, Comparison of solutions to a three-dimensional black-oil reservoir simulation problem (includes associated paper 9741), Journal of Petroleum Technology, 33 (1981), pp. 13-25.

[57] J. Papež, U. RÜde, M. Vohralík, And B. Wohlmuth, Sharp algebraic and total a posteriori error bounds for hp finite elements via a multilevel approach. In preparation, 2017.

[58] W. Prager and J. L. Synge, Approximations in elasticity based on the concept of function space, Quart. Appl. Math., 5 (1947), pp. 241-269.

[59] O. Ricois, Vision générale du simulateur ARCEOR, tech. rep., IFPEN, 2011.

[60] J. E. Roberts and J.-M. Thomas, Mixed and hybrid methods, in Handbook of Numerical Analysis, Vol. II, North-Holland, Amsterdam, 1991, pp. 523-639.

[61] A. Sboui, J. Jaffré, And J. Roberts, A composite mixed finite element for hexahedral grids, SIAM J. Sci. Comput., 31 (2009), pp. 2623-2645.

[62] H. A. VAN DER VorST, Bi-CGSTAB: a fast and smoothly converging variant of Bi-CG for the solution of nonsymmetric linear systems, SIAM J. Sci. Statist. Comput., 13 (1992), pp. 631-644.

[63] M. VohraLík, A posteriori error estimates for lowest-order mixed finite element discretizations of convection-diffusion-reaction equations, SIAM J. Numer. Anal., 45 (2007), pp. 1570-1599. 
[64] - Residual flux-based a posteriori error estimates for finite volume and related locally conservative methods, Numer. Math., 111 (2008), pp. 121-158.

[65] — Unified primal formulation-based a priori and a posteriori error analysis of mixed finite element methods, Math. Comp., 79 (2010), pp. 2001-2032.

[66] M. Vohralík And M. F. Wheeler, A posteriori error estimates, stopping criteria, and adaptivity for two-phase flows, Comput. Geosci., 17 (2013), pp. 789-812.

[67] M. Vohralík And B. I. Wohlmuth, Mixed finite element methods: implementation with one unknown per element, local flux expressions, positivity, polygonal meshes, and relations to other methods, Math. Models Methods Appl. Sci., 23 (2013), pp. 803-838.

[68] M. F. Wheeler and I. Yotov, A multipoint flux mixed finite element method, SIAM J. Numer. Anal., 44 (2006), pp. 2082-2106.

[69] M. Wolff, Y. CaO, B. Flemisch, R. Helmig, and B. Wohlmuth, Multi-point flux approximation L-method in 3D: numerical convergence and application to two-phase flow through porous media, Radon Ser. Comput. Appl. Math., De Gruyter, 12 (2013), pp. 39-80.

[70] S. Yousef, A posteriori error estimates and adaptivity based on stopping criteria and adaptive mesh refinement for multiphase and thermal flows. Application to steam-assisted gravity drainage, Ph.D. thesis, Université Pierre et Marie Curie - Paris VI, 2013. 